\title{
Uncertainty Assessments of 2D and Axisymmetric Hypersonic Shock Wave - Turbulent Boundary Layer Interaction Simulations at Compression Corners
}

\author{
Peter A. Gnoffo* and Scott A. Berry ${ }^{\dagger}$ \\ NASA Langley Research Center, Hampton, VA 23681-2199 \\ John W. Van Norman ${ }^{\ddagger}$ \\ Analytical Mechanics Associates, Inc., Hampton, VA 23681-2199
}

\begin{abstract}
This paper is one of a series of five papers in a special session organized by the NASA Fundamental Aeronautics Program that addresses uncertainty assessments for CFD simulations in hypersonic flow. Simulations of a shock emanating from a compression corner and interacting with a fully developed turbulent boundary layer are evaluated herein. Mission relevant conditions at Mach 7 and Mach 14 are defined for a pre-compression ramp of a scramjet powered vehicle. Three compression angles are defined - the smallest to avoid separation losses and the largest to force a separated flow engaging more complicated flow physics. The Baldwin-Lomax and the Cebeci-Smith algebraic models, the oneequation Spalart-Allmaras model with the Catrix-Aupoix compressibility modification and two-equation models including Menter SST, Wilcox $k-\omega$ 98, and Wilcox $k-\omega 06$ turbulence models are evaluated. Each model is fully defined herein to preclude any ambiguity regarding model implementation. Comparisons are made to existing experimental data and Van Driest theory to provide preliminary assessment of model form uncertainty. A set of coarse grained uncertainty metrics are defined to capture essential differences among turbulence models. Except for the inability of algebraic models to converge for some separated flows there is no clearly superior model as judged by these metrics. A preliminary metric for the numerical component of uncertainty in shock - turbulent-boundary-layer interactions at compression corners sufficiently steep to cause separation is defined as $55 \%$. This value is a median of differences with experimental data averaged for peak pressure and heating and for extent of separation captured in new, grid-converged solutions presented here. This value is consistent with existing results in a literature review of hypersonic shock - turbulent-boundary-layer interactions by Roy and Blottner and with more recent computations of MacLean.
\end{abstract}

\section{Nomenclature}

Some variable names are specific to a particular turbulence model. If they are not found in this list they will be defined in the section they are encountered.

Roman symbols

$\begin{array}{ll}A^{+} & \text {modified Van Driest coefficient [Eq. 10] } \\ B_{q} & \text { dimensionless heating, } q_{w} /\left(\rho_{w} c_{p} u_{\tau} T_{w}\right) \\ c & \text { speed of sound [m/s] } \\ c_{p} & \text { heat capacity at constant pressure }[\mathrm{J} / \mathrm{kg}-\mathrm{K}] \\ C_{f} & \text { skin friction coefficient, } \tau_{w} /\left(\frac{1}{2} \rho_{e} u_{e}^{2}\right)\end{array}$

\footnotetext{
*Senior Research Engineer, Aerothermodynamics Branch; AIAA Fellow

${ }^{\dagger}$ Senior Research Engineer, Aerothermodynamics Branch; AIAA Associate Fellow

¥Senior Project Engineer, AIAA Member
} 


\section{Roman symbols}

$\bar{C}_{f}$

$D_{s}$

$D_{t}$

$e$

$e_{v, s}^{*}$

E

$\mathcal{F}$

$h$

H

$\mathcal{H}$

$\hat{I}_{s}$

$k$

$l$

$M_{t}$

$M_{\tau}$

$\mathcal{M}$

$n$

$\dot{n}_{e^{-}, s}$

$n^{+}$

$p$

$P^{+}$

$\mathcal{P}$

$P r_{t}$

$q$

$Q_{\text {rad }}$

$r_{\text {prod,lim }}$

$R e_{\theta}$

$\bar{R} e_{\theta}$

$S_{c_{t}}$

$S_{i j}$

$\bar{S}_{i j}$

$\hat{S}_{i j}$

$t_{i j}$

T

$u$

$u_{i}, u_{j}$

$u_{\tau}$

$U$

$U^{+}$

$\dot{w}_{s}$

$x$

$x_{i}, x_{j}$

$y$

$y^{+}$ incompressible friction coefficient

effective diffusion coefficient for species $s\left[\mathrm{~m}^{2} / \mathrm{s}\right]$

turbulent diffusion coefficient $\left[\mathrm{m}^{2} / \mathrm{s}\right]$

static energy $[\mathrm{J} / \mathrm{kg}]$

vibrational energy per unit mass evaluated at temperature $T$, $[\mathrm{J} / \mathrm{kg}]$

metric of difference between computation and experiment

vorticity function [Eq. 17]

static enthalpy $[\mathrm{J} / \mathrm{kg}]$

total enthalpy, $[\mathrm{J} / \mathrm{kg}]$

Heaviside step function

first ionization energy of species $s[\mathrm{~J} / \mathrm{kg}$-mole]

turbulent kinetic energy $[\mathrm{J} / \mathrm{kg}]$

mixing length [Eq. 9]

turbulence Mach number, $\sqrt{2 k} / c$

Mach number based on friction velocity, $u_{\tau} / c_{w}$

molecular weight $[\mathrm{kg} / \mathrm{kg}-\mathrm{mole}]$

coordinate orthogonal to wall $[\mathrm{m}]$

molar rate of production of species $s$ by electron impact ionization $\left[\mathrm{kg}-\mathrm{mole} / \mathrm{m}^{3} \mathrm{~s}\right.$ ]

$\rho u_{\tau} n / \mu$, normalized distance to wall

pressure $\left[\mathrm{N} / \mathrm{m}^{2}\right]$

transformed pressure gradient, [Eq. 11]

production term in turbulent kinetic energy equation [Eq. 27]

turbulent Prandtl number

heat transfer rate $\left[\mathrm{W} / \mathrm{m}^{2}\right]$

radiative energy transfer rate $\left[\mathrm{J} / \mathrm{m}^{3}-\mathrm{s}\right]$

maximum allowed value of production to destruction ratio [Eq. 27]

momentum thickness Reynolds number

incompressible momentum thickness Reynolds number

turbulent Schmidt number, $\mu_{t} / \rho D_{t}$

mean-strain-rate tensor, $\frac{1}{2}\left(\frac{\partial u_{i}}{\partial x_{j}}+\frac{\partial u_{j}}{\partial x_{i}}\right)$

zero-trace version of mean-strain-rate tensor, $S_{i j}-\frac{1}{3} \frac{\partial u_{k}}{\partial x_{k}} \delta_{i j}$

$S_{i j}-\frac{1}{2} \frac{\partial u_{k}}{\partial x_{k}} \delta_{i j}$

molecular stress tensor, $2 \mu \bar{S}_{i j}$

temperature $[\mathrm{K}]$

velocity $[\mathrm{m} / \mathrm{s}]$

velocity component in $i$ and $j$ directions, respectively $[\mathrm{m} / \mathrm{s}]$

friction velocity, $\sqrt{\tau_{w} / \rho_{w}}$

velocity tangent to wall, $[\mathrm{m} / \mathrm{s}]$

$u / u_{\tau}$, dimensionless velocity

mass rate of production of species $s\left[\mathrm{~kg} / \mathrm{m}^{3}-\mathrm{s}\right]$

distance along wall (flat plate), coordinate in streamwise direction [m]

coordinates in $i$ and $j$ directions, respectively [m]

distance normal to wall (flat plate), coordinate orthogonal to $x[\mathrm{~m}]$

$\rho u_{\tau} y / \mu$, normalized distance (flat plate) 


$\begin{array}{ll}\text { Greek symbols } & \\ \gamma_{i} & \text { intermittency function, Eq. 15] } \\ \delta & \text { boundary layer thickness, }[\mathrm{m}] \\ \delta_{k} & \text { velocity thickness, [Eq. 14] } \\ \epsilon^{+} & \text {eddy viscosity, } \mu_{t} / \mu \\ \epsilon_{i}^{+} & \text {inner layer eddy viscosity } \\ \epsilon_{o}^{+} & \text {outer layer eddy viscosity } \\ \eta & \text { molecular translational-rotational conductivity }[\mathrm{J} / \mathrm{K}-\mathrm{m}-\mathrm{s}] \\ \eta_{t} & \text { turbulent translational-rotational conductivity }[\mathrm{J} / \mathrm{K}-\mathrm{m}-\mathrm{s}] \\ \eta_{v} & \text { molecular vibrational-electronic conductivity }[\mathrm{J} / \mathrm{K}-\mathrm{m}-\mathrm{s}] \\ \eta_{v t} & \text { turbulent vibrational-electronic conductivity }[\mathrm{J} / \mathrm{K}-\mathrm{m}-\mathrm{s}] \\ \mu & \text { viscosity }[\mathrm{kg} / \mathrm{m}-\mathrm{s}] \\ \nu & \text { eddy viscosity, } \mu / \rho \\ \rho & \text { density }\left[\mathrm{kg} / \mathrm{m}^{3}\right] \\ \tau & \text { shear }\left[\mathrm{N} / \mathrm{m}^{2}\right] \\ \tau_{i j} & \text { Reynolds stress tensor [Eq. } 28] \\ \chi_{s} & \text { mole fraction of species } s \\ \Omega_{k} & \left(\frac{\partial u_{k-1}}{\partial x_{k+1}}-\frac{\partial u_{k+1}}{\partial x_{k-1}}\right) \\ \text { Subscripts } & \\ e & \text { at edge of boundary layer } \\ e- & \text { electron } \\ i & \text { component in } i \text { direction } \\ j & \text { component in } j \text { direction } \\ m o l . & \text { molecules } \\ s & \text { species index } \\ t & \text { turbulent value } \\ v & \text { vibrational-electronic energy component } \\ V D & \text { parameter used in Van Driest transform } \\ w & \text { conditions at wall } \\ \infty & \text { reference condition in free stream } \\ & \end{array}$

\section{Introduction}

The Fundamental Aero Program (FAP) has a goal of reducing uncertainties in hypersonic flow simulation by $50 \%$. The target goal started as a subjective assessment of the simulation needs by NASA researchers looking at diverse challenges and rolled up into a single metric. The FAP has now been tasked to define the metrics by which success or failure is judged.

The definition of simulation uncertainty in hypersonics has little meaning without specifying free stream conditions and configuration geometry. Different physical phenomena engage as a function of total enthalpy, atmospheric composition, vehicle size, and vehicle shape. Consequently, five mission relevant simulations have been defined to characterize simulation uncertainties for specific conditions. The focus here is on hypersonic cruise conditions for a scram-jet with shock wave - turbulent boundary layer interaction at a compression corner. We include compression corner angles that are sufficiently steep to cause separation of an incoming turbulent boundary layer (something not wanted for a scramjet) because it provides significant challenges to computational simulation. Furthermore, the ability to predict the maximum compression angle that does not separate the boundary layer provides opportunity to open design space and shorten the running length to the inlet.

Quantifying uncertainty reduction further requires a well-defined initial simulation capability from which new modeling improvements can be measured. Consequently, we focus on turbulence models that have seen wide application in production codes through 2006 - the approximate time frame when FAP goals were first 
announced. By "production" codes we refer to codes like LAURA, ${ }^{1,2}$ DPLR, ${ }^{3}$ and Vulcan ${ }^{4}$ that are routinely used for simulation of hypersonic flows within NASA. The models used in these codes in the 2006 time frame include algebraic, and various one- and two-equation turbulence models. Detached eddy simulations (DES), ${ }^{5}$ large eddy simulations (LES), ${ }^{6}$ and direct numerical simulations (DNS) ${ }^{7}$ in the hypersonic domain are not considered in the 2006 baseline. Advances were clearly being made in these approaches ${ }^{8}$ in that time frame but they were not yet routine or robust components of production codes for hypersonic simulations. In like manner, these production codes did not have routine capability to predict transition location using a coupled stability analysis like STABL ${ }^{9}$ or LASTRAC.3d. ${ }^{10}$ Specification of transition location, if implemented at all, generally was based on $\operatorname{Re}_{\theta} / M_{e}$ correlations. The dependence of the shock wave turbulent boundary layer interaction (SWTBLI) at a compression corner as a function of distance from the upstream transition location is not well understood. Presumably, if the transition location (tripped or natural) is close to the SWTBLI then the unsteady nature of transition will feed an additional source of unsteadiness into the interaction. A transition location far upstream (hundreds of boundary-layer thicknesses) feeding a fully developed, turbulent boundary layer profile into the SWTBLI is therefore a prerequisite for experimental data sets used to establish uncertainty. Even with a fully developed, incoming turbulent boundary layer experimental results show that shock oscillation is of inherent nature in the SWTBLI with separation and that shock oscillation is considered to be the consequence of the coherent structures in the separated region. ${ }^{11}$

This uncertainty definition process is started by building on the 2006 review of turbulence models for hypersonic flows by Roy and Blottner, ${ }^{12}$ which in turn sought to update the comprehensive review of hypersonic shock/turbulent boundary-layer interaction experiments published in 1991 by Settles and Dodson. ${ }^{13,14}$ Knight et al. ${ }^{15}$ have assembled a complementary review for supersonic flow including interactions associated with compression corners, shock impingement, single fin, and double fins. ${ }^{15}$ however, the Mach numbers $(2.3,3)$ and adiabatic boundary conditions for the compression corner problems do not address the hypersonic domain, especially the role of a cold wall boundary. More recent experiments with computation by Reinartz et al. ${ }^{16}$ examined wall temperature effect in a shock tunnel flow at Mach 7.7 and 7.4 but did not have fully developed turbulent flow on the flat plate leading to the 15 degree compression corner. Holden et.al. ${ }^{17}$ have provided new contributions to the computational and experimental analysis of the canonical SWTBLI compression corner problems in the LENS facility at CUBRC. These new data sets are used herein because they feature long models with natural transition far upstream of the compression corner at hypersonic conditions and the compression surface is sufficiently long to allow a plateau in pressure downstream of the separation without a terminating expansion to suppress growth of the recirculation.

The paper is structured as follows. We first define a mission relevant test problem including free stream conditions for which the simulation uncertainty will be characterized. The governing equations and turbulence models are then documented to remove any ambiguity of how they are formulated. A suite of uncertainty quantification metrics are introduced based on a collection of simulations reviewed by Roy and Blottner. ${ }^{12}$ This suite of coarse grained uncertainty metrics is defined to easily capture their values from figures in the literature. Relevant experimental data on compression corners are then reviewed and new simulations are executed with a variety of models and codes representative of the state of the art in 2006 as explained earlier. The new metrics are calculated for both existing and new simulations and a representative value is proposed. Finally, we return to the mission relevant test problem (which has no experimental reference data), execute new simulations including gas chemistry perturbations in order to assess an overall uncertainty metric. Two appendices are also provided to present verification and validation data on a simpler flat plate problem.

\section{Definition of Mission Relevant Test Problem}

The development of a hypersonic cruise vehicle using air breathing propulsion is deemed a high priority mission within NASA's Fundamental Aero Program (FAP). Two trajectory points are defined for a representative mission ${ }^{18}$ in Table 1 below. The geometry for the compression ramp leading to the inlet is a two-dimensional, double-wedge approximation (Fig. 1) that is loosely based on an X-43A configuration discussed by Berry et al. ${ }^{19}$ The two-dimensional configuration has a 4.5 degree wedge with a $12 \mathrm{ft} .(3.66 \mathrm{~m})$ running length from the sharp leading edge to the compression corner. The second section provides another 5.5 deg. compression (10 deg. total) with another 6 feet $(1.83 \mathrm{~m})$ running length to the end of the body. (A blunted leading edge with $1 \mathrm{~cm}$ radius was rejected in favor of the sharp leading edge; it exhibited entropy effects that influenced laminar separation at the corner.) 
Table 1. Trajectory Points for Mission Relevant Problem

\begin{tabular}{cccccc}
\hline$M_{\infty}$ & $V_{\infty}, \mathrm{m} / \mathrm{s}$ & $\rho_{\infty}, \mathrm{kg} / \mathrm{m}^{3}$ & alt., $\mathrm{km}$ & $T, \mathrm{~K}$ & $T_{\text {wall }}, \mathrm{K}$ \\
\hline 7 & 2086 & $4.40110^{-2}$ & 24.4 & 220.94 & 500 \\
14 & 4418 & $9.81110^{-3}$ & 33.7 & 246.93 & 500 \\
\hline
\end{tabular}

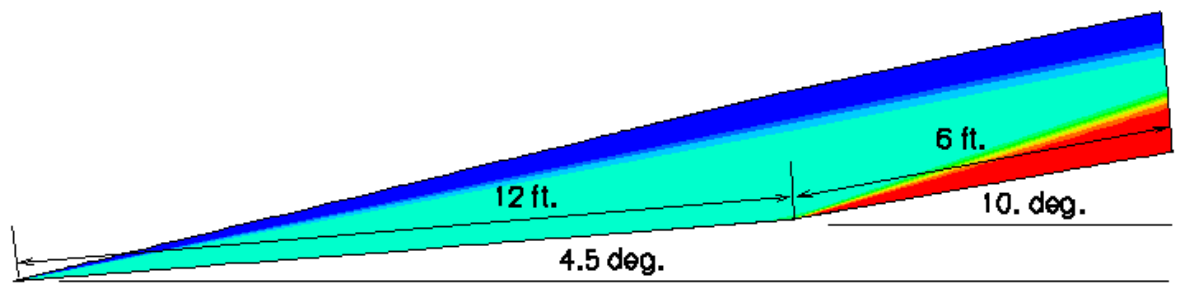

Figure 1. Pressure contours over mission relevant configuration. (blue low, red high)

The 5.5 deg. compression is not intended to separate the flow at the corner. Two additional configurations with a 30 deg. compression (34.5 deg. total) and a $35 \mathrm{deg}$. compression (39.5 deg. total) will also be analyzed. In these cases, a separation zone is desired because simulation of a separated zone is more challenging and because the ability to identify an incipient separation condition is important for understanding design space constraints. These configurations and conditions are deemed canonical problems with relevance to a high priority mission of interest to FAP.

\section{Conservation Equations}

The conservation equations with effects of RANS turbulent dissipation are provided below. Turbulent kinetic energy, if modeled by a 2-eq. formulation, is included in the total energy conservation equation. Note that source terms for chemical reaction and thermal relaxation do not include any modification associated with real turbulent fluctuations.

\section{A. Species Conservation}

$$
\frac{\partial\left(\rho_{s}\right)}{\partial t}+\frac{\partial\left(\rho_{s} u_{j}\right)}{\partial x_{j}}=\frac{\partial}{\partial x_{j}}\left[\left(\rho D_{s}+\frac{\mu_{t} \mathcal{M}_{s}}{S_{c_{t}} \mathcal{M}}\right) \frac{\partial \chi_{s}}{\partial x_{j}}\right]+\dot{w}_{s}
$$

B. Momentum Conservation

$$
\frac{\partial\left(\rho u_{i}\right)}{\partial t}+\frac{\partial\left(\rho u_{j} u_{i}\right)}{\partial x_{j}}=-\frac{\partial p}{\partial x_{i}}+\frac{\partial}{\partial x_{j}}\left[t_{j i}+\tau_{j i}\right]
$$

\section{Total Energy Conservation}

$$
\begin{aligned}
\frac{\partial}{\partial t}\left[\rho\left(e+\frac{u_{i} u_{i}}{2}+k\right)\right] & +\frac{\partial}{\partial x_{j}}\left[\rho u_{j}\left(h+\frac{u_{i} u_{i}}{2}+k\right)\right]= \\
& +\frac{\partial}{\partial x_{j}}\left[u_{i}\left(t_{j i}+\tau_{j i}\right)\right]+\frac{\partial}{\partial x_{j}}\left[\sum_{s}\left(\rho D_{s}+\frac{\mu_{t} \mathcal{M} s_{s}}{S_{c_{t}} \mathcal{M}}\right) h_{s} \frac{\partial \chi_{s}}{\partial x_{j}}\right] \\
& +\frac{\partial}{\partial x_{j}}\left[\left(\eta+\eta_{t}\right) \frac{\partial T}{\partial x_{j}}+\left(\eta_{v}+\eta_{v t}\right) \frac{\partial T_{v}}{\partial x_{j}}\right]+\frac{\partial}{\partial x_{j}}\left[\left(\mu+\sigma_{k} \frac{\rho k}{\omega}\right) \frac{\partial k}{\partial x_{j}}\right]-Q_{\text {rad }}
\end{aligned}
$$

where

$$
\eta_{t}=\frac{\eta}{\eta+\eta_{v}} \frac{\mu_{t}}{c_{p} P r_{t}}, \quad \eta_{v t}=\frac{\eta_{v}}{\eta+\eta_{v}} \frac{\mu_{t}}{c_{p} P r_{t}}
$$




\section{Vibrational-Electronic Energy Conservation}

$$
\begin{aligned}
\frac{\partial \rho e_{v}}{\partial t} & +\frac{\partial \rho u_{j} e_{v}}{\partial x_{j}}=-p_{e-} \frac{\partial u_{j}}{\partial x_{j}} \\
& +\frac{\partial}{\partial x_{j}}\left[\sum_{s}\left(\rho D_{s}+\frac{\mu_{t} \mathcal{M}_{s}}{S_{c_{t}} \mathcal{M}}\right) h_{v, s} \frac{\partial \chi_{s}}{\partial x_{j}}\right]+\frac{\partial}{\partial x_{j}}\left[\left(\eta_{v}+\eta_{v t}\right) \frac{\partial T_{v}}{\partial x_{j}}\right] \\
& +\sum_{s=\text { mol. }} \rho_{s} \frac{\left(e_{v, s}^{*}-e_{v, s}\right)}{\tau_{s}}+2 \rho_{e-} \frac{3}{2} \bar{R}\left(T-T_{v}\right) \sum_{s \neq e-} \dot{n}_{e-, s} \hat{I}_{s}+\sum_{s=\text { mol. }} \dot{w}_{s} \hat{D}_{s}-Q_{\text {rad }}
\end{aligned}
$$

\section{Turbulence Models}

The turbulence models used herein are fully documented in this section to avoid any ambiguity in the value of constants or the definition of source terms. In this regard, a current, on-line resource on turbulence models may be found at http://turbmodels.larc.nasa.gov.

\section{A. Algebraic Models}

The algebraic formulations of eddy viscosity use the Gupta modifications ${ }^{20}$ to the models of Cebeci-Smith ${ }^{21}$ and Baldwin-Lomax. ${ }^{22}$ The model formulations are repeated below to maintain a complete record of all turbulence models used within LAURA ${ }^{1}$ for this study. The original references provide a more complete explanation of the model derivations.

The algebraic eddy viscosity is given by

$$
\epsilon^{+}=\left\{\begin{array}{cc}
\epsilon_{i}^{+} & n \leq n_{\text {crossover }} \\
\epsilon_{o}^{+} & n>n_{\text {crossover }}
\end{array}\right.
$$

where $n_{\text {crossover }}$ is the value of $n$ where $\epsilon_{i}^{+}=\epsilon_{o}^{+}$.

\section{Cebeci-Smith}

The inner eddy viscosity in the baseline model is defined as

$$
\begin{aligned}
\epsilon_{i}^{+} & =\frac{\rho l^{2}}{\mu}\left|\frac{\partial U}{\partial n}\right| \\
l & =0.4 n\left[1-\exp \left(-n^{+} / A^{+}\right)\right] \\
A^{+} & =\frac{26}{\sqrt{1-11.8 P^{+}}} \\
P^{+} & =-\frac{\mu}{\rho^{2} u_{\tau}^{3}}\left(\frac{\partial p}{\partial s}\right)_{e}
\end{aligned}
$$

An alternative definition of $A^{+}$suggested by Gupta $^{20}$ and found to yield good agreement with turbulent heating data on blunt bodies for cold hypersonic flow is

$$
A^{+}=\frac{26}{\sqrt{\left|\tau / \tau_{w}\right|}}
$$

The approximate equivalence of $\tau / \tau_{w}$ and $1-11.8 P^{+}$can be derived by numerically integrating the streamwise momentum equation across the sublayer from $y^{+}=0$ to $y^{+}=11.8$ as discussed by Cebeci. ${ }^{23}$ The definition of $A^{+}$in Eq. 10 removes the singularity that can occur in the case of separated flow not to mention the implicit dependence of $\tau$ on $\epsilon_{i}^{+}$. However, strongly expanding flows (as around the corner of a blunt

body) can lead to negative values of the argument in Eq. 10. Results presented herein use Eq. 12 unless otherwise noted. 
The outer eddy viscosity is

$$
\begin{aligned}
\epsilon_{o}^{+} & =\frac{0.0168 \rho U_{e} \delta_{k} \gamma_{i}}{\mu} \\
\delta_{k} & =\int_{0}^{\delta}\left(1-\frac{U}{U_{e}}\right) d n \\
\gamma_{i} & =\left[1+5.5\left(\frac{n}{\delta}\right)^{6}\right]^{-1}
\end{aligned}
$$

The coordinates $n$ and $s$ are orthogonal and tangent to the wall and $U$ is the velocity component in the $s$ direction. The boundary layer thickness $\delta$ is defined as the value of $n$ where $\frac{H}{H_{\infty}}=0.995$. This definition needs to be adjusted in the case of shock layer radiation where the total enthalpy is not conserved across the inviscid layer.

\section{Baldwin-Lomax}

The inner eddy viscosity is computed as in the Cebeci-Smith model using Equations 8, 9, and 12. The outer eddy viscosity is

$$
\begin{aligned}
\epsilon_{o}^{+} & =\frac{0.0168 C_{C P} \rho F_{\text {wake }} F_{\text {Kleb }}}{\mu} \\
\mathcal{F}(n) & =n\left|\frac{\partial U}{\partial n}\right|\left[1-\exp \left(-n^{+} / A^{+}\right)\right] \\
F_{\text {wake }} & =n_{\text {max }} \mathcal{F}_{\text {max }} \\
F_{\text {Kleb }} & =\left[1+5.5\left(\frac{0.3 n}{n_{\text {max }}}\right)^{6}\right]^{-1}
\end{aligned}
$$

Here $C_{C P}=1.6, \mathcal{F}_{\text {max }}$ is the maximum value of the vorticity function $\mathcal{F}$ across the outer layer and $n_{\max }$ is the value of $n$ where $\mathcal{F}\left(n_{\max }\right)=\mathcal{F}_{\max }$. Note that the thin-layer approximation $\left|\frac{\partial U}{\partial n}\right| \approx|\Omega|$ is used to define $\epsilon^{+}$.

\section{B. One-Equation Model - Spalart-Allmaras}

The compressible form of the Spalart-Allmaras ${ }^{24}$ model developed by Catrix and Aupoix ${ }^{25}$ is defined

$$
\frac{\partial(\rho \hat{\nu})}{\partial t}+\frac{\partial\left(\rho u_{j} \hat{\nu}\right)}{\partial x_{j}}=c_{b 1} \Omega \rho \hat{\nu}-c_{\omega 1} f_{\omega} \rho\left(\frac{\hat{\nu}}{d}\right)^{2}+\frac{1}{\sigma} \frac{\partial}{\partial x_{j}}\left(\mu \frac{\partial \hat{\nu}}{\partial x_{j}}\right)+\frac{1}{\sigma} \frac{\partial}{\partial x_{j}}\left(\sqrt{\rho} \hat{\nu} \frac{\partial \sqrt{\rho} \hat{\nu}}{\partial x_{j}}\right)+\frac{c_{b 2}}{\sigma} \frac{\partial \sqrt{\rho} \hat{\nu}}{\partial x_{i}} \frac{\partial \sqrt{\rho} \hat{\nu}}{\partial x_{i}}
$$

The turbulent eddy viscosity is defined

$$
\mu_{t}=\rho \hat{\nu} f_{\nu 1}, \quad f_{\nu 1}=\frac{\chi_{\nu}^{3}}{\chi_{\nu}^{3}+c_{\nu 1}^{3}}, \quad \chi_{\nu}=\frac{\hat{\nu}}{\nu}
$$

Other constants and functions required to complete this model are:

$$
\begin{gathered}
c_{b 1}=0.1355, \quad c_{b 2}=0.622, \quad c_{\nu 1}=7.1, \quad \sigma=\frac{2}{3}, \quad \kappa=0.41 \\
c_{\omega 1}=\frac{c_{b 1}}{\kappa^{2}}+\frac{1+c_{b 2}}{\sigma} \quad c_{\omega 2}=0.3, \quad c_{\omega 3}=2 \\
f_{\omega}=g\left[\frac{1+c_{\omega 3}^{6}}{g^{6}+c_{\omega 3}^{6}}\right]^{1 / 6}, \quad g=r=c_{\omega 2}\left(r^{6}-r\right), \quad r=\min \left[\frac{\hat{\nu}}{\hat{\mathcal{S}} \kappa^{2} d^{2}}, 10\right], \quad \hat{\mathcal{S}}=\Omega+\frac{\hat{\nu}}{\kappa^{2} d^{2}} f_{\nu 2}
\end{gathered}
$$




\section{Two-Equation Models}

The two-equation models used herein share the following conservation law form for turbulent kinetic energy $k$ and the specific dissipation rate $\omega .^{26}$

$$
\begin{aligned}
\frac{\partial(\rho k)}{\partial t}+\frac{\partial\left(\rho u_{j} k\right)}{\partial x_{j}} & =\mathcal{P}-\beta_{c}^{*} \rho \omega k+\frac{\partial}{\partial x_{j}}\left[\left(\mu+\sigma_{k} \frac{\rho k}{\omega}\right) \frac{\partial k}{\partial x_{j}}\right] \\
\frac{\partial(\rho \omega)}{\partial t}+\frac{\partial\left(\rho u_{j} \omega\right)}{\partial x_{j}} & =\frac{\gamma}{\nu^{*}} \mathcal{P}-\beta_{c} \rho \omega^{2}+\frac{\partial}{\partial x_{j}}\left[\left(\mu+\sigma_{\omega} \frac{\rho k}{\omega}\right) \frac{\partial \omega}{\partial x_{j}}\right]+\phi \frac{\partial k}{\partial x_{j}} \frac{\partial \omega}{\partial x_{j}}
\end{aligned}
$$

All of the two-equation models considered herein define the production term $\mathcal{P}$ as

$$
\begin{aligned}
\mathcal{P} & =\min \left(\tau_{i j} \frac{\partial u_{i}}{\partial x_{j}}, r_{\text {prod,lim }} \beta_{c}^{*} \rho \omega k\right) \\
\tau_{i j} & =2 \mu_{t} \bar{S}_{i j}-\frac{2}{3} \rho k \delta_{i j}
\end{aligned}
$$

The production term is limited by some factor $r_{\text {prod,lim }}$ (defaults to 20 in LAURA) times the destruction term to provide more realistic values of turbulent kinetic energy behind shocks.

\section{Compressibility Corrections}

Rumsey ${ }^{27}$ has reviewed the impact of compressibility corrections on turbulent, hypersonic boundary layers on flat plates. He investigates the claim that "... compressibility corrections are not required for hypersonic boundary layer flows ... for a wide range of Mach numbers and wall-temperature boundary conditions" and ultimately notes that compressibility corrections derived for free shear layers do not work well for boundary layers and vice versa. Compressibility corrections were originally derived to account for the reduction in a free shear layer growth due to compressibility. ${ }^{28}$ In dealing with separated flows, it seems logical that some type of compressibility correction is needed though an optimal implementation is not obvious. The Sarkar/Zeman ${ }^{29,30}$ compressibility modifications to define $\beta_{c}^{*}$ and $\beta_{c}$ from the baseline model values of $\beta^{*}$ and $\beta$ are applied here using Wilcox's model to define $F\left(M_{t}\right)$.

$$
\beta_{c}^{*}=\beta^{*}\left[1+\xi^{*} f\left(M_{t}\right)\right], \quad \beta_{c}=\beta-\beta^{*} \xi^{*} F\left(M_{t}\right)
$$

with

$$
\xi^{*}=2, \quad M_{t_{0}}=\frac{1}{4}, \quad F\left(M_{t}\right)=\left[M_{t}^{2}-M_{t_{0}}^{2}\right] \mathcal{H}\left(M_{t}-M_{t_{o}}\right)
$$

In general, compressibility corrections are not applied $\left(F\left(M_{t}\right)=0\right)$ unless specifically noted.

2. $k-\omega 2006^{26}$

The turbulent eddy viscosity is defined as

$$
\mu_{t}=\frac{\rho k}{\hat{\omega}}, \quad \hat{\omega}=\max \left[\omega, C_{\text {lim }} \sqrt{\frac{2 \bar{S}_{i j} \bar{S}_{i j}}{\beta^{*}}}\right], \quad C_{\text {lim }}=\frac{7}{8}
$$

Other constants and functions required to complete this model are:

$$
\begin{gathered}
\sigma_{k}=0.6, \quad \sigma_{\omega}=0.5, \quad \gamma=\frac{13}{25}, \quad \nu^{*}=\frac{k}{\omega}, \quad \beta^{*}=0.09, \quad \beta_{0}=0.0708, \quad \beta=\beta_{0} f_{\beta}, \\
f_{\beta}=\frac{1+85 \chi_{\omega}}{1+100 \chi_{\omega}}, \quad \chi_{\omega}=\left|\frac{\Omega_{i j} \Omega_{k j} \hat{S}_{k i}}{\left(\beta^{*} \omega\right)^{3}}\right|, \quad \phi=\frac{\rho \sigma_{d}}{\omega}, \quad \sigma_{d}=\left\{\begin{array}{ccc}
0 & \text { for } \quad \frac{\partial k}{\partial x_{j}} \frac{\partial \omega}{\partial x_{j}} \leq 0 \\
\frac{1}{8} & \text { for } \quad \frac{\partial k}{\partial x_{j}} \frac{\partial \omega}{\partial x_{j}}>0
\end{array}\right.
\end{gathered}
$$

Ghost cell values for $k$ and $\omega$ behind a no-slip wall are defined as

$$
k_{g c}=0, \quad \omega_{g c}=\frac{6 \nu_{1}}{\beta_{0} d^{2}}
$$

where subscript 1 indicates the value at the bounding cell center and $d$ is the distance from the wall to the bounding cell center. 
3. $k-\omega 1998^{26}$

The turbulent eddy viscosity is defined as

$$
\mu_{t}=\frac{\rho k}{\omega}
$$

Other constants and functions required to complete this model are:

$$
\begin{aligned}
& \sigma_{k}=0.5, \quad \sigma_{\omega}=0.5, \quad \gamma=\frac{13}{25}, \quad \nu^{*}=\frac{k}{\omega}, \quad \phi=0 \\
& \beta_{0}=0.0708, \quad \beta=\beta_{0} f_{\beta}, \quad f_{\beta}=\frac{1+70 \chi_{\omega}}{1+80 \chi_{\omega}}, \quad \chi_{\omega}=\left|\frac{\Omega_{i j} \Omega_{k j} S_{k i}}{\left(\beta_{0}^{*} \omega\right)^{3}}\right| \\
& \beta_{0}^{*}=0.09, \quad \beta^{*}=\beta_{0}^{*} f_{\beta^{*}}, \quad f_{\beta^{*}}=\left\{\begin{array}{cc}
1 & \text { for } \quad \chi_{k} \leq 0 \\
\frac{1+680 \chi_{k}}{1+400 \chi_{k}} & \text { for } \quad \chi_{k}>0
\end{array}, \quad \chi_{k}=\frac{1}{\omega^{3}} \frac{\partial k}{\partial x_{j}} \frac{\partial \omega}{\partial x_{j}}\right.
\end{aligned}
$$

Ghost cell values for $k$ and $\omega$ behind a no-slip wall are defined as

$$
k_{g c}=0, \quad \omega_{g c}=\frac{6 \nu_{1}}{\beta_{0} d^{2}}
$$

where subscript 1 indicates the value at the bounding cell center and $d$ is the distance from the wall to the bounding cell center.

\section{Menter-SST $T^{31}$}

The turbulent eddy viscosity is defined as

$$
\mu_{t}=\frac{\rho a_{1} k}{\max \left(a_{1} \omega, \Omega F_{2}\right)}, \quad a_{1}=0.31
$$

Other constants and functions required to complete this model are:

$$
\begin{gathered}
F_{1}=\tanh \left(\arg _{1}^{4}\right), \quad \arg g_{1}=\min \left[\max \left(\frac{\sqrt{k}}{\beta^{*} \omega d}, \frac{500 \nu}{d^{2} \omega}\right), \frac{4 \rho \sigma_{\omega 2} k}{C D_{k \omega} d^{2}}\right], \quad C D_{k \omega}=\max \left(\frac{2 \rho \sigma_{\omega 2}}{\omega} \frac{\partial k}{\partial x_{j}} \frac{\partial \omega}{\partial x_{j}}, 10^{-20}\right) \\
F_{2}=\tanh \left(\arg g_{2}^{2}\right), \quad \arg _{2}=\max \left(2 \frac{\sqrt{k}}{\beta^{*} \omega d}, \frac{500 \nu}{d^{2} \omega}\right) \\
\begin{array}{cll}
\sigma_{k}=F_{1} \sigma_{k 1}+\left(1-F_{1}\right) \sigma_{k 2}, & \sigma_{k 1}=0.85, & \sigma_{k 2}=1.0 \\
\sigma_{\omega}=F_{1} \sigma_{\omega 1}+\left(1-F_{1}\right) \sigma_{\omega 2}, & \sigma_{\omega 1}=0.5, & \sigma_{\omega 2}=0.856 \\
\beta=F_{1} \beta_{1}+\left(1-F_{1}\right) \beta_{2}, & \beta_{1}=0.075, & \beta_{2}=0.0828 \\
\gamma=F_{1} \gamma_{1}+\left(1-F_{1}\right) \gamma_{2}, & \gamma_{1}=\beta_{1} / \beta^{*}-\sigma_{\omega 1} \kappa^{2} / \sqrt{\beta^{*}}, & \gamma_{2}=\beta_{2} / \beta^{*}-\sigma_{\omega 2} \kappa^{2} / \sqrt{\beta^{*}} \\
\beta^{*}=0.09, & \nu^{*}=\nu_{t}, \quad \phi=2\left(1-F_{1}\right) \frac{\rho \sigma_{\omega 2}}{\omega}, & \kappa=0.41
\end{array}
\end{gathered}
$$

Ghost cell values for $k$ and $\omega$ behind a no-slip wall are defined as

$$
k_{g c}=0, \quad \omega_{g c}=\frac{60 \nu_{1}}{\beta_{1} d^{2}}
$$

where subscript 1 indicates the value at the bounding cell center and $d$ is the distance from the wall to the bounding cell center. 


\section{Uncertainty Metrics / System Response Quantities}

\section{A. Simulation Uncertainty}

As will be seen in the next section, a significant variation in computed results is observed as a function of turbulence model for shock wave / turbulent boundary layer interactions (SWTBLI) that induce separation. Simple, coarse grain uncertainty metrics for system response quantities (SRQ in the terminology of Oberkampf and $\mathrm{Roy}^{32}$ ) are proposed here to capture essential differences between computation and experiment. As additional experimental data is gathered and turbulence models are winnowed and converge, finer grain metrics are required to finish the evolution of turbulence models for hypersonic flows.

The coarse grain metrics focus on surface measurements in the the pre-compression region, separated region (if it exists experimentally), and the post-compression region including the maximum measured values of surface pressure, heating, and shear. Off-body measurements are deferred for use in defining the fine grain metrics. The metrics in this section are computed without any regard for experimental uncertainty. The increments associated with experimental uncertainty will be computed in the next sub-section.

In the post-compression region (see for example the region where $x>1.04$ in Fig. 8) metrics are proposed that compare the maximum measured values of pressure, heating and shear to the maximum computed values. The maximum values need not occur at the same physical location. Thus,

$$
E\left(f_{\text {max }}\right)=\left(\frac{f_{\text {post }, c f d, \text { max }}-f_{\text {post }, \text { exp }, \text { max }}}{f_{\text {post }, \text { exp }, \text { max }}}\right) \times 100
$$

where $f$ represents $p, q$, or $\tau$. If there is no local maximum in the post-compression region the values immediately following the jump will be used.

The metric in the pre-compression region (see for example the region where $x<0.95$ in Fig. 8 ) is tuned to check that inflow conditions setting up the interaction are correctly simulated. Select a subset of $N_{\text {pre }}$ measurements just upstream of the interaction to form an average value of $f_{\text {pre,exp }}$ and an average value of location $x_{\text {pre,exp }}$

$$
\begin{aligned}
f_{\text {pre }, \text { exp }} & =\frac{1}{N_{\text {pre }}} \sum_{n=1}^{N_{\text {pre }}} f_{\text {exp }}\left(x_{n, \text { exp }}\right) \\
x_{\text {pre }, \text { exp }} & =\frac{1}{N_{\text {pre }}} \sum_{n=1}^{N_{\text {pre }}} x_{n, \exp }
\end{aligned}
$$

The subjective nature of choosing $N_{\text {pre }}$ upstream measurements is acknowledged. The definition is in keeping with the coarse grain metric definitions that are intended to be easily defined by inspection of plotted results. If there is a significant difference between the measurements that make up the average relative to the measurement uncertainty in this domain then this metric should be abandoned. Assuming an appropriate average value can be defined then the pre-compression metrics are given by

$$
E\left(f_{\text {pre }}\right)=\left(\frac{f_{\text {pre }, \text { cfd }}-f_{\text {exp }}\left(x_{\text {pre }, \text { exp }}\right)}{f_{\text {pre }, \exp }}\right) \times 100
$$

The metric in the separation region (see for example the region where $0.95<x<1.04$ in Fig. 8) is defined at the midpoint between the experimentally measured separation point and the corner location, $x_{\text {sep }, \text { mid }}=\left(x_{\text {sep }, \exp }+x_{\text {corner }}\right) / 2$.

$$
E\left(f_{\text {sep }}\right)=\left(\frac{f_{c f d}\left(x_{\text {sep }, \text { mid }}\right)-f_{\text {exp }}\left(x_{\text {sep }, \text { mid }}\right)}{f_{\text {exp }}\left(x_{\text {sep }, \text { mid }}\right)}\right) \times 100
$$

Note that the separation metric is defined even if the simulation shows no separation. Finally, a metric for the extent of separation is defined

$$
E(L)=\left(\frac{x_{\text {sep }, c f d}-x_{\text {sep }, \text { exp }}}{x_{\text {sep }, \text { exp }}-x_{\text {corner }}}\right) \times 100
$$

The separation metric is defined at the location where the pressure and heating show an abrupt increase in slope. All of these metrics return negative values if the computed variable under-predicts the measured 
variable. The value of $E(L)$ is 100 if the experiment shows any separation and the computation shows no separation. Many of the metrics recorded here were extracted from published figures where there was difficulty in reading differences to better than a couple of percent. For this reason, all metrics are consistently rounded to the nearest $5 \%$.

A single metric may be defined from these coarse grain definitions to provide a simple measure of the current state-of-the-art. This metric is strictly used to provide an easily defined target (though still a challenging goal) for the Fundamental Aeronautics Program (FAP) to assess improvements in hypersonic simulation capability. This metric will be introduced after reviewing the results of multiple simulations here and in the literature.

\section{B. Experimental Uncertainty}

The CUBRC experimental data ${ }^{17,33}$ is the prime source of experimental data here because the large models and test conditions produce natural transition well upstream of the interaction region. Of all the available experimental data sets, the CUBRC data best covers the Mach number range and total enthalpy range of the mission relevant problem as shown in Fig. 2. Uncertainty in measured pressure is given as $\pm 3 \%$. Uncertainty in measured heating is given as $\pm 5 \%$. Uncertainty in measured shear was not discussed. Other sources ${ }^{34,35}$ estimate shear measurement uncertainty at $\pm 7 \%$ to $\pm 12 \%$ with $\pm 10 \%$ assumed for subsequent calculations. Uncertainties in free stream dynamic pressure $\left(\rho_{\infty} V^{2}{ }_{\infty} / 2\right)$ and stagnation point enthalpy $\left(\approx V^{2}{ }_{\infty} / 2\right)$ are $\pm 5 \%$. Using dimensional analysis, the uncertainty in free stream energy flux $\left(\rho_{\infty} V^{3}{ }_{\infty} / 2\right)$ is $1.05 \times 1.05^{0.5}-1$ or $\pm 7.6 \%$. If one assumes that the changes in pressure coefficient, skin friction coefficient, and heat transfer coefficient at any point on the body are small compared to changes in these free stream quantities then the total experimental uncertainties due to calibration and free stream uncertainties are estimated to be: $\Delta p_{\exp }=\sqrt{3^{2}+5^{2}}= \pm 5.8 \%, \Delta \tau_{\exp }=\sqrt{10^{2}+5^{2}}= \pm 11.2 \%$, and $\Delta q_{\exp }=\sqrt{5^{2}+7.6^{2}}= \pm 9.1 \%$. The uncertainty for separation length $\Delta L_{\text {exp }}$ is taken as the distance between gauges at the separation point divided by the experimental separation length times 100. For Run 54 this uncertainty is computed as $\Delta L_{\text {exp }}=(0.32 / 2.54) 100=12.6 \%$. These estimates do not include measurement errors associated with high frequency unsteadiness relative to gauge response time - possibly an issue inside the separation zone. Nor do they include any errors associated with flow non-uniformity in time and space or model alignment.

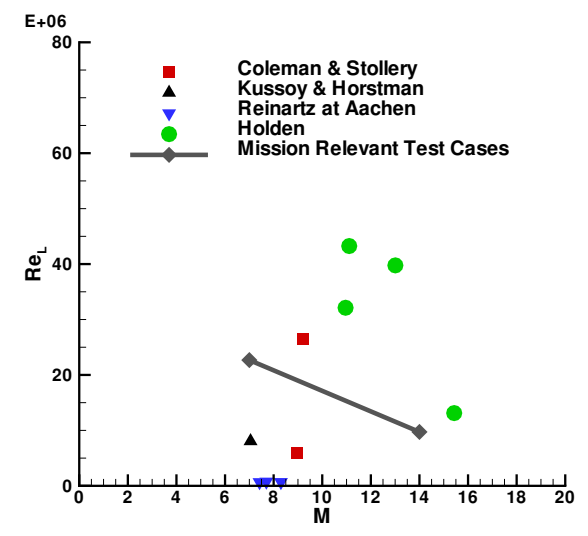

(a) Reynolds number versus Mach number

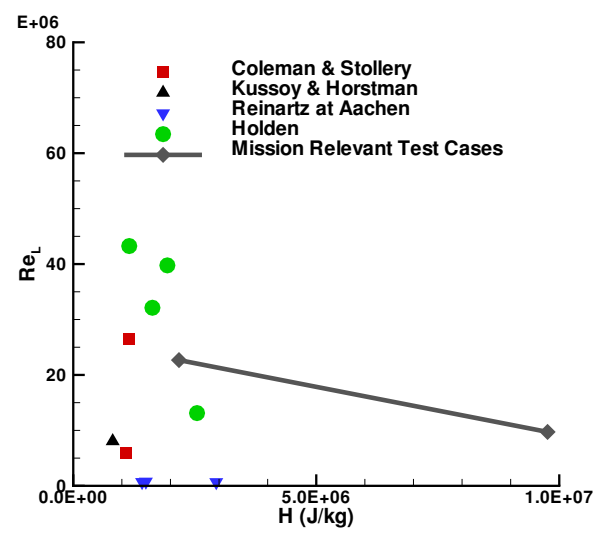

(b) Reynolds number versus total enthalpy

Figure 2. Relation of experimental data sets (symbols) to mission relevant problem trajectory points (connected by line).

Some other experimental cases covered in the review by Roy and Blottner are also presented to provide a more diverse set of simulations from which uncertainty metrics may be derived. We assume experimental errors in these sources are at least as large as those discussed above from CUBRC. A more complete discussion of the definition of uncertainty metrics follows in the next section, after having the opportunity to observe the primary ways in which the simulation and experiment differ. 


\section{Test Cases with Experimental Data for Compression Corners}

In the following figures the flow is in the $x-y$ plane defined by $z=0$. The $x$ coordinate is aligned with the flow direction in the free stream. The wall shear stress is computed as $\tau=\cos (\theta) \tau_{x}+\sin (\theta) \tau_{y}$ where $\theta$ is the inclination angle of the wall relative to the $x$ axis.

In all cases the turbulence model is engaged from the leading edge of the model even if the transition location is defined in the experimental data. There is an implicit assumption that fully developed turbulent flow is present upstream of the interaction region in all of the simulations. The possible effects of transition location and transition length are accepted as contributions to the modeling uncertainty.

\section{A. CUBRC Compression Corner - Run 54}

Experimental data for a turbulent boundary layer at Mach 11.3 on a flat plate followed by a 36 deg. ramp obtained at CUBRC $^{17}$ are used as the first validation check for the suite of turbulence models applied to shock / turbulent boundary layer interactions at compression corners. The geometry is presented in Fig. 3. The baseline grid uses 264 cells along the plate and 94 cells normal to the plate. Two additional grids are also utilized which provide a factor of two (fine grid) and 4 (very fine grid) additional resolution in both coordinate directions relative to the baseline grid. The near wall region prior to separation is resolved with $y^{+} \approx .015$, achieved by specifying a cell Reynolds number equal to 0.1 in the initial grid generation process. Inflow boundary conditions are: $V_{\infty}=1,769 \mathrm{~m} / \mathrm{s}, \rho_{\infty}=0.08246 \mathrm{~kg} / \mathrm{m}^{3}, T_{\infty}=61 \mathrm{~K}$ and $\alpha=0$ deg.. These inflow conditions are very close to those of the flat plate case used as a verification and validation check in Appendix B. Surface boundary conditions are no-slip with $T_{w}=300 \mathrm{~K}$. Supersonic outflow boundary conditions are extrapolated from the interior. The test gas, air, is treated as calorically perfect unless otherwise noted. Molecular transport properties are computed from Sutherlands law.

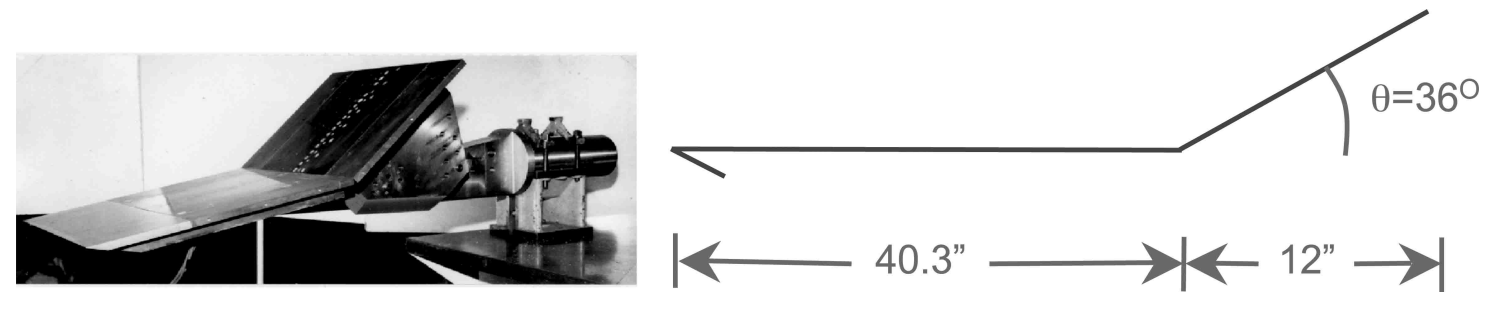

Figure 3. Turbulent wedge geometry for CUBRC Run $54 .^{17}$

Figure 4 compares experimental data for pressure and heating along the surface to simulations using various turbulence models with LAURA. The solid line result in each figure shows the simulation and symbols show the experiment. A log scale is used to provide equivalent resolution of pre- and post- shock levels of heating and pressure. In general the percent difference between simulated and measured heating in this compression corner case is equivalent to that same difference measured for the flat plate in Appendix $\mathrm{B}$ although the log scale makes comparisons look better. For example, in the baseline $k-\omega(2006)$ in Fig. 4 (d), the average measured heating on gauges with $0.8<x<0.96$ is $4.86 \mathrm{~W} / \mathrm{cm}^{2}$. The average simulation value over this same range is $6.43 \mathrm{~W} / \mathrm{cm}^{2}$, an over-prediction of $32 \%$. For the flat plate the average measured heating over the same range is $5.40 \mathrm{~W} / \mathrm{cm}^{2}$ and the average simulation value is 7.18 $\mathrm{W} / \mathrm{cm}^{2}$, an over-prediction of $33 \%$. The coarse grained metrics from Eqs. $43-48$ for this figure are captured in Table 2 .

All of the algebraic models failed to converge for this case. The separation point moved far upstream and large scale, unsteady motion ensued within the separation zone. The Cebeci-Smith model using the damping coefficient given by either Eq. 10 or Eq. 12 behaved similarly. The experimental data suggests existence of a rather flat heating plateau. The remaining 2-eq. models under-predict the extent of separation if no compressibility correction is applied. A general trend is observed for the simulations without compressibility correction (Fig. 4 (b-d)) such that larger predicted separation zones produce greater overshoots in post-shock peak heating rate and, to a lesser extent, pressure. Heating overshoots in these cases vary from approximately $100 \%$ to $25 \%$. The downstream asymptotic heating levels never recover with difference varying from approximately $100 \%$ to $20 \%$. Application of a compressibility correction (with $M_{t 0} \geq 0.4$ to avoid conditions that disturb agreement with the van Driest distributions noted in Appendix B) produce 
a separation extent in better agreement with experiment — see Fig. 4 (e-f). Pressure levels across the separation zone are in good agreement with measured data. Predicted heating levels in the separation zone are lower than measurements and no heating plateau is observed.

Comparisons to experimental data for shear are presented in Fig. 5 using a linear scale to capture positive and negative values. In addition to over-predicting extent of separation as noted earlier the Spalart-Allmaras model under-predicts peak shear by $150 \%$. The Menter SST model has best agreement with the shear data on the baseline grid. All of the models tend to under-predict the measured shear while over-predicting the measured heating in the post-compression domain.

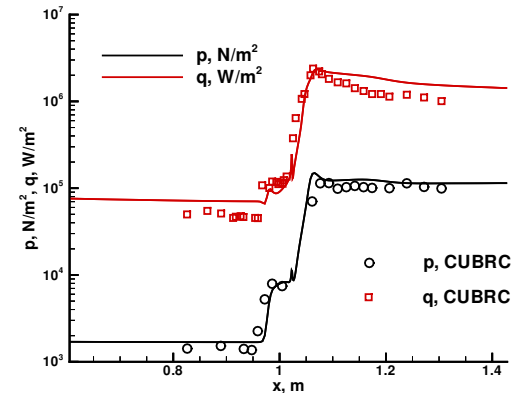

(a) 1-eq: Spalart-Allmaras

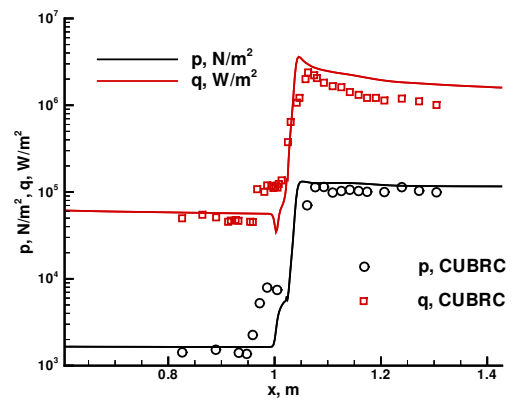

(c) 2-eq.: $k-\omega(1998)$

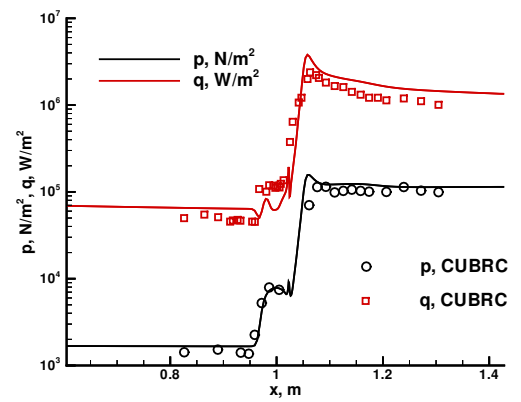

(e) 2-eq: $k-\omega(2006)\left(\mathrm{CC} M_{t 0}=0.5\right)$

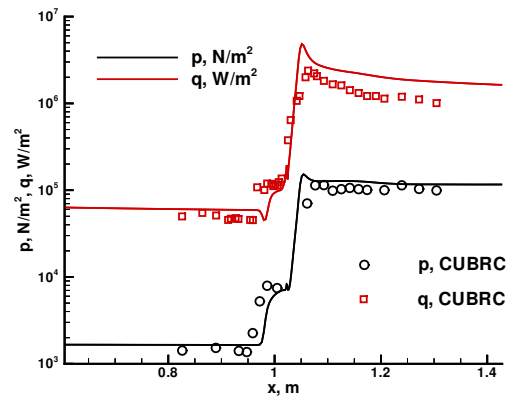

(b) 2-eq.: Menter SST

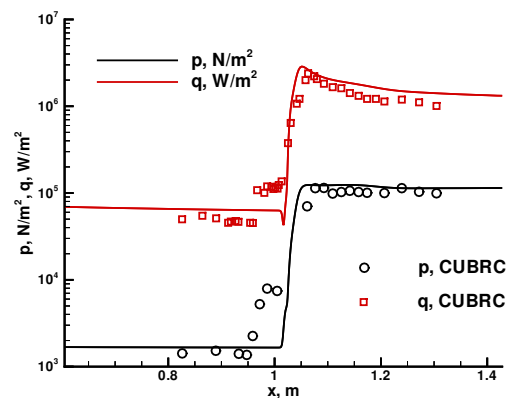

(d) 2-eq: $k-\omega(2006)$

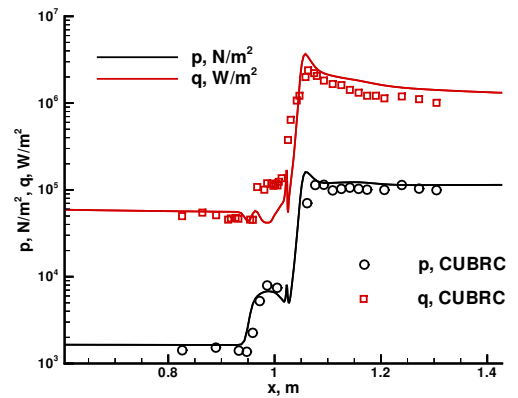

(f) 2-eq: $k-\omega(2006)\left(\mathrm{CC} M_{t 0}=0.4\right)$

Figure 4. Pressure and heating distributions over a compression corner at Mach 11.3 computed with 1- and 2-eq. turbulence models on baseline grid (264 x 94). Algebraic models failed to converge for this case.

All of these compression corner results were computed on grids that yield grid converged results for the flat plate. However, the resolution of a separated region prior to a compression corner generally requires finer resolution. A previous validation study for laminar flows ${ }^{36}$ shows that onset of separation tends to be under-predicted (smaller recirculation) until a grid converged solution is achieved. Based on this observation, 
the $k-\omega$ (2006) model with various compressibility corrections is retested on finer grids (fine: (528 x 188) and very fine $(1056 \times 376))$. The recirculation region is resolved with approximately (148 x 228) cells on the very fine grid.

The surface pressure and heating distributions on these uniformly refined grids are presented in Fig. 6 and the surface shear distributions are presented in Fig. 7. The simulations without compressibility correction fail to show any significant separation even on the finest grid. The simulation using $M_{t 0}=0.7$ provides the best overall agreement with measured pressure, heating, and shear levels (Fig. $6(\mathrm{~b}, \mathrm{f})$ and Fig. 7 (b,f)). The extent of separation appears to be grid converged. The heating rate overshoot on the post compression side is approximately $130 \%$. Some additional structure appears in the heating distribution on the finest grid that was not evident on the fine grid. The fine grid heating in the plateau region is in good agreement with measurement but an approximately $70 \%$ overshoot is evident on the finest grid. The coarse grained metrics from Eqs. 43 - 48 for Fig. 6 are captured in Table 3.

Table 2. Coarse-Grained Uncertainty Metrics for Fig. 4

\begin{tabular}{cccccccc}
\hline Model & $E\left(q_{\max }\right)$ & $E\left(p_{\max }\right)$ & $E\left(q_{\text {sep }}\right)$ & $E\left(p_{\text {sep }}\right)$ & $E\left(q_{\text {pre }}\right)$ & $E\left(p_{\text {pre }}\right)$ & $E(L)$ \\
\hline SA,$(264 \times 94)$ & 0 & 40 & 50 & 20 & 40 & 10 & 20 \\
SST, (264 x 94) & 100 & 35 & 35 & 10 & 15 & 10 & 20 \\
$k-\omega 98,(264 \times 94)$ & 60 & 15 & -220 & -110 & 15 & 10 & 80 \\
$k-\omega 06,(264 \times 94)$ & 20 & 10 & -230 & -90 & 20 & 10 & 95 \\
$k-\omega 06.5,(264 \times 94)$ & 70 & 40 & -100 & 5 & 20 & 10 & 0 \\
$k-\omega 06.4,(264 \times 94)$ & 60 & 50 & -100 & -25 & 10 & 10 & -5 \\
\hline
\end{tabular}

Table 3. Coarse-Grained Uncertainty Metrics for Fig. 6 and associated, un-plotted results from Vulcan and DPLR

\begin{tabular}{|c|c|c|c|c|c|c|c|c|}
\hline Code & Model & $E\left(q_{\max }\right)$ & $E\left(p_{\max }\right)$ & $E\left(q_{s e p}\right)$ & $E\left(p_{\text {sep }}\right)$ & $E\left(q_{\text {pre }}\right)$ & $E\left(p_{\text {pre }}\right)$ & $E(L)$ \\
\hline LAURA & $k-\omega 06,(528 \times 188)$ & 20 & 10 & -230 & -90 & 15 & 10 & 100 \\
\hline LAURA & $k-\omega 06.7,(528 \times 188)$ & 120 & 80 & 0 & 40 & 15 & 10 & 0 \\
\hline LAURA & $k-\omega 06.6,(528 \times 188)$ & 110 & 80 & -30 & 40 & 15 & 10 & -10 \\
\hline LAURA & $k-\omega 06.5,(528 \times 188)$ & 110 & 90 & -60 & -30 & 15 & 10 & -60 \\
\hline LAURA & $k-\omega 06,(1056 \times 376)$ & 25 & 10 & -230 & -90 & 15 & 10 & 100 \\
\hline LAURA & $k-\omega 06.7,(1056 \times 376)$ & 130 & 80 & 30 & 50 & 15 & 10 & 0 \\
\hline Vulcan & $k-\omega 06,(1056 \times 376)$ & 60 & 35 & 30 & 35 & 15 & 10 & -50 \\
\hline Vulcan & $k-\omega 06$, unl & 60 & 40 & 40 & 10 & 15 & 10 & 0 \\
\hline Vulcan & $k-\omega 98,(1056 \times 376)$ & 60 & 50 & 40 & 55 & 15 & 10 & -55 \\
\hline Vulcan & $k-\omega 98$, unl & 5 & 15 & -70 & -155 & 15 & 10 & 100 \\
\hline Vulcan & Men. SST, (1056x376) & 70 & 60 & 60 & 70 & 15 & 10 & -190 \\
\hline Vulcan & Men. SST, unl & 75 & 60 & 60 & 60 & 15 & 10 & -140 \\
\hline DPLR & Men. SST, $(266 \times 96)$ & 40 & 45 & 10 & 0 & 15 & 10 & -35 \\
\hline DPLR & Men. SST, mod. stress lim. & 45 & 35 & 0 & -5 & 35 & 10 & 5 \\
\hline
\end{tabular}

In Fig. 8 we focus on a single turbulence model (Menter - SST) but consider how the simulation results may vary as a result of different implementations within a code (Fig. 8-a) or between codes (Fig. 8-b). The progression of models study was motivated by an observation that the extent of separation observed in DPLR results (V4.01.1) for the Menter-SST was over-predicted compared to experimental data ${ }^{17}$ but was underpredicted in the LAURA implementation (Fig. 4-b). (See a related study in Appendix B for a flat plate.) The DPLR solution was rerun using the LAURA grid and perfect-gas model. ${ }^{37}$ No significant change in the extent of separation was observed. The remaining steps made changes to the LAURA implementation to make it more like the DPLR implementation. The DPLR implementation of the conservation laws for $k$ and 


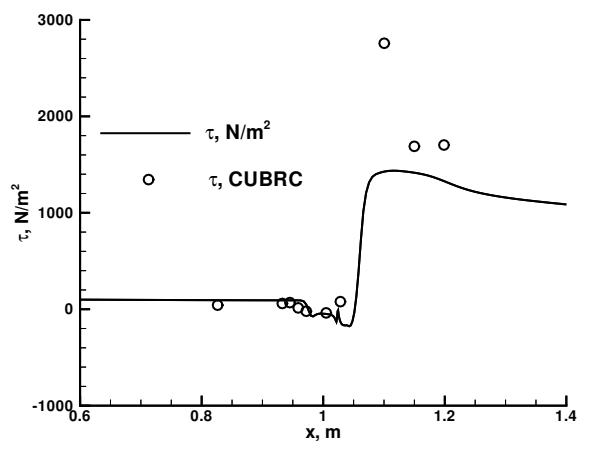

(a) 1-eq: Spalart-Allmaras

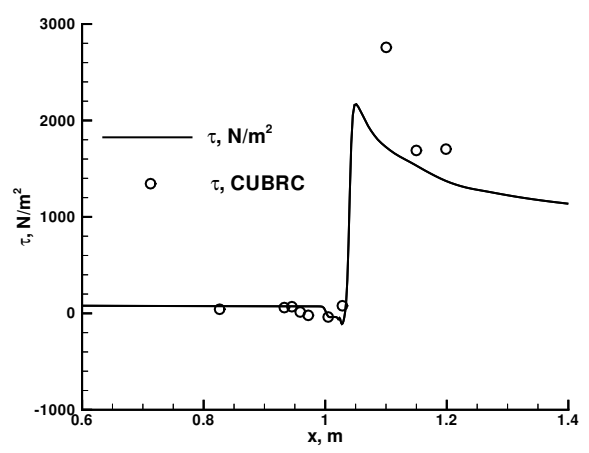

(c) 2-eq.: $k-\omega(1998)$

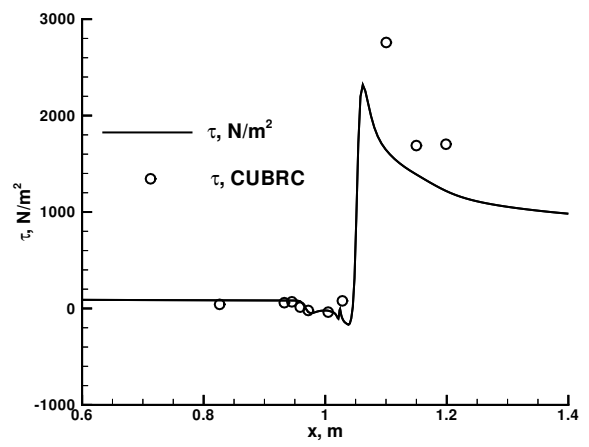

(e) 2-eq: $k-\omega(2006)\left(\mathrm{CC} M_{t 0}=0.5\right)$

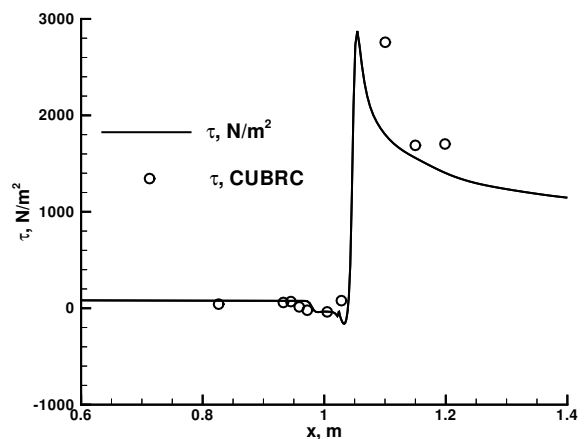

(b) 2-eq.: Menter SST

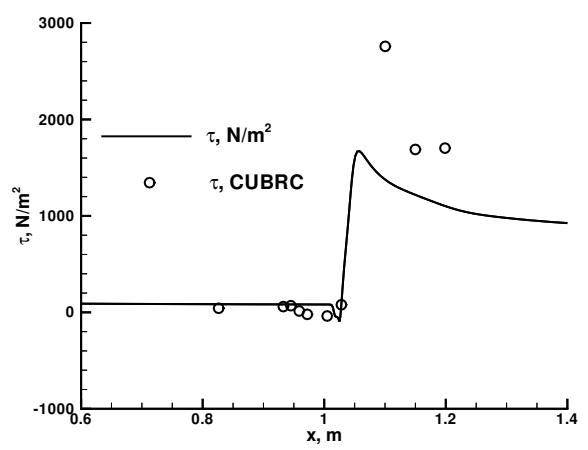

(d) 2-eq: $k-\omega(2006)$

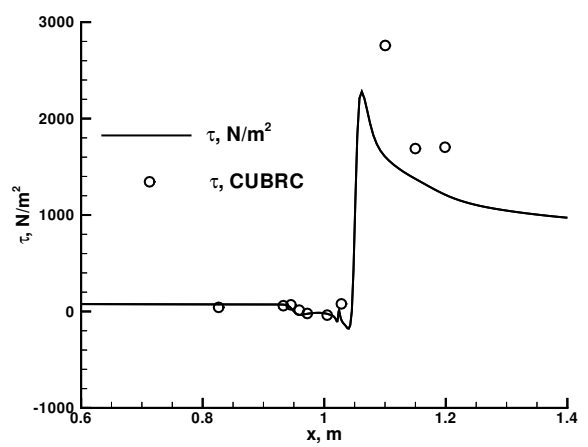

(f) 2-eq: $k-\omega(2006)\left(\mathrm{CC} M_{t 0}=0.4\right)$

Figure 5. Shear force distributions over a compression corner at Mach 11.3 computed with 1- and 2-eq. turbulence models on baseline grid (264 x 94). Algebraic models failed to converge for this case while showing massive, unsteady separation. 


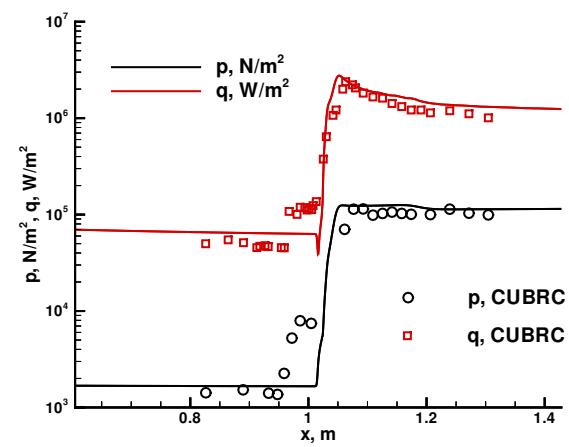

(a) No compressibility correction, on fine grid (528 x 188)

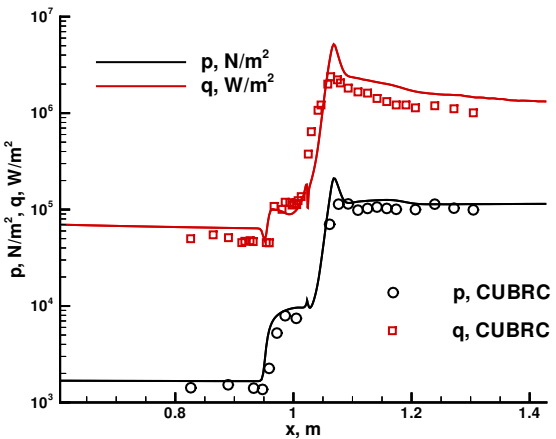

(c) CC $M_{t 0}=0.6$, on fine grid $(528 \times 188)$

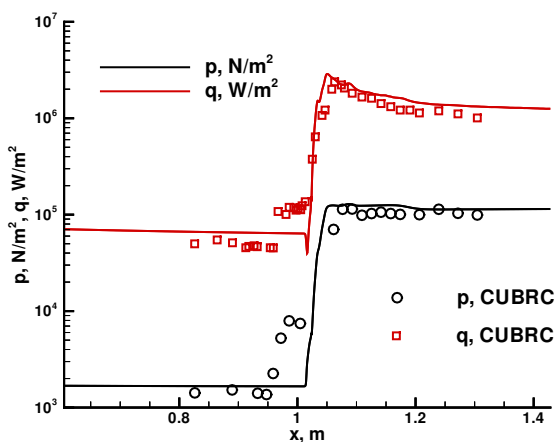

(e) No compressibility correction, on very fine grid $(1056 \times 376)$

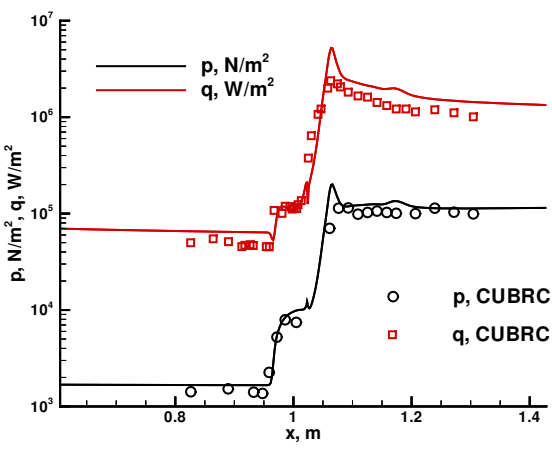

(b) CC $M_{t 0}=0.7$, on fine grid $(528 \times 188)$

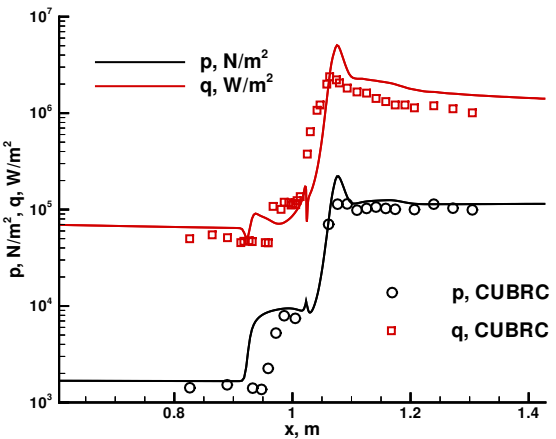

(d) $\mathrm{CC} M_{t 0}=0.5$, on fine grid $(528 \times 188)$

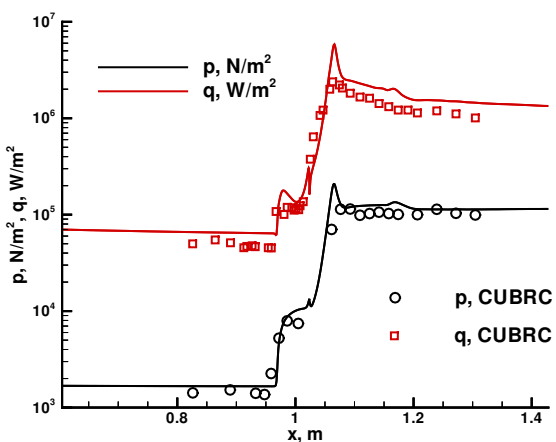

(f) $\mathrm{CC} M_{t 0}=0.7$, on very fine grid $(1056 \times 376)$

Figure 6. Pressure and heating distributions over a compression corner at Mach 11.3 computed with $k-\omega$ (2006) turbulence model on refined grids. 


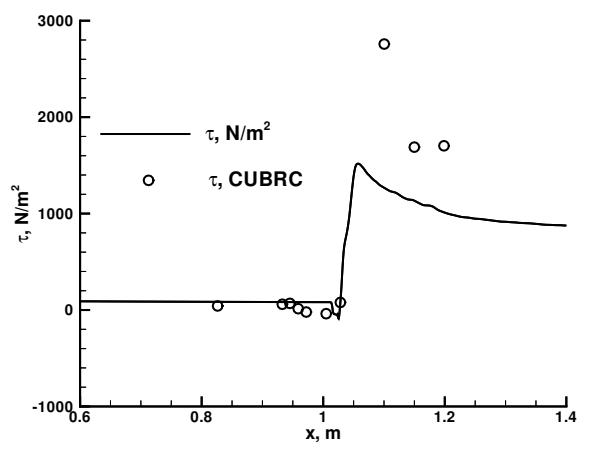

(a) No compressibility correction, fine grid (528 x 188)

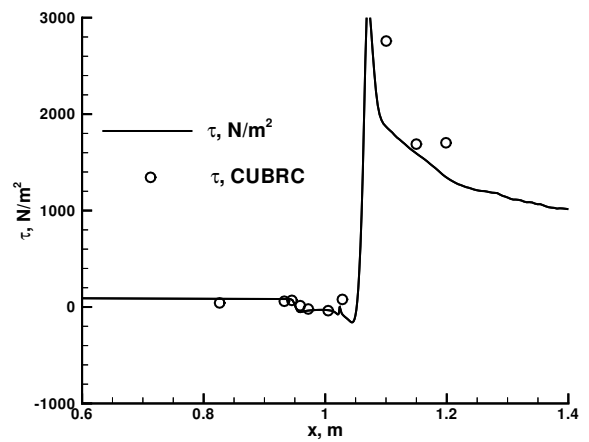

(c) $\mathrm{CC} M_{t 0}=0.6$, fine grid $(528 \times 188)$

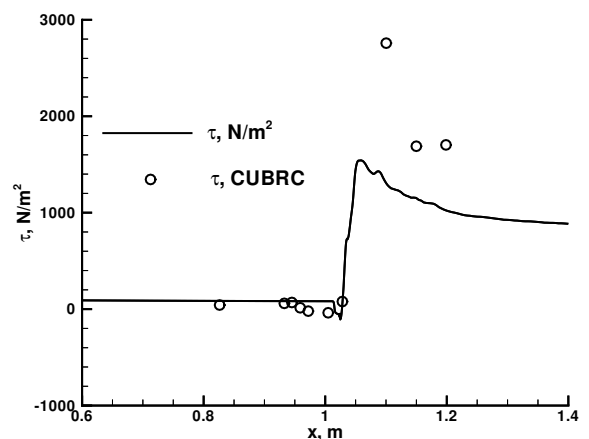

(e) No compressibility correction, on very fine grid $(1056 \times 376)$

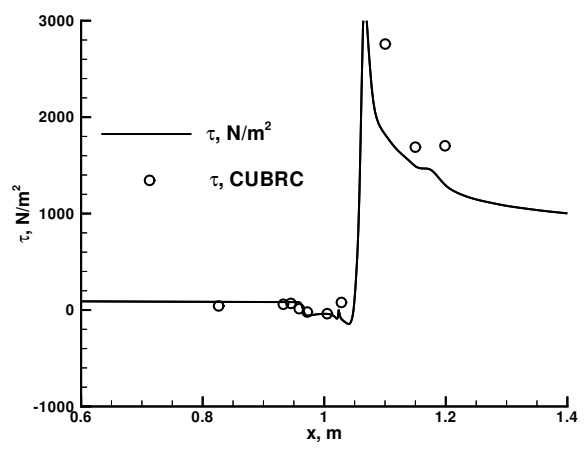

(b) CC $M_{t 0}=0.7$, fine grid $(528 \times 188)$

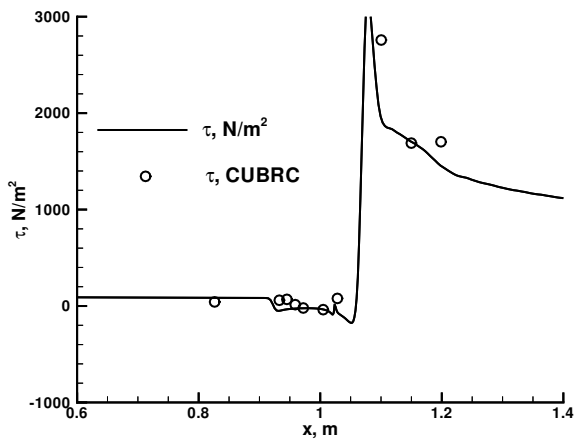

(d) CC $M_{t 0}=0.5$, fine grid $(528 \times 188)$

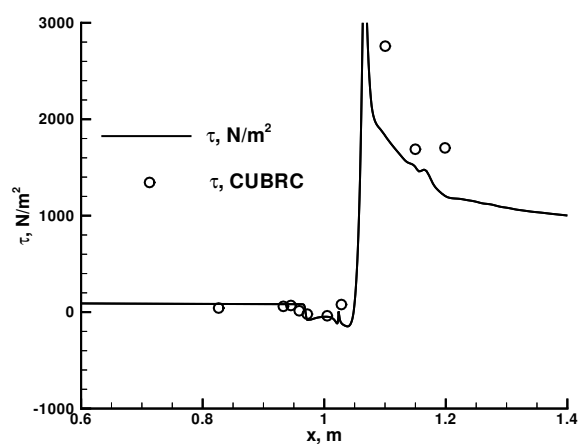

(f) $\mathrm{CC} M_{t 0}=0.7$, on very fine grid $(1056 \times 376)$

Figure 7. Shear force distributions over a compression corner at Mach 11.3 computed with $k-\omega$ (2006) turbulence model on refined grids. 
$\omega$ employs a loosely coupled formulation with 1st-order spatial accuracy in the convection terms. Baseline LAURA uses strong coupling of all equations so that convective terms are all second-order accurate away from any limiting conditions. LAURA was modified so that only the turbulence equations were formulated with first-order convection. This change caused a slight decrease in heating levels in the separation zone but no change in the extent of separation was observed. The next change to LAURA reset the free stream values of $k$ and $\omega$ to match DPLR; this modification caused no significant change to the pressure or heating. The last modification recognizes that baseline LAURA computes the production term $\mathcal{P}=\tau_{i j} \frac{\partial u_{i}}{\partial x_{j}}$ (see Eq. 28) while baseline DPLR uses $\mathcal{P}=\mu_{t}\left(\Omega_{x}^{2}+\Omega_{y}^{2}+\Omega_{z}^{2}\right)$. This modification caused over-prediction of the extent of separation and made the result more like DPLR - though it now predicts more separation than DPLR.

The pressure and heating distributions from three different codes using their standard implementation of the Menter-SST model are presented in Fig. 8-b. The LAURA results (black and red) are computed on the fine grid. No significant changes are expected if computed on the very fine grid based on the earlier grid convergence study in Figs. 6 a versus e, $\mathrm{b}$ versus $\mathrm{f}$ and in Figs. 7 a versus e and $\mathrm{b}$ versus $\mathrm{f}$. The LAURA results show a small decrease in the extent of separation for the 5-species air model compared to perfect gas. The maximum temperature in the flow is approximately $1,000 \mathrm{~K}$ so dissociation is not expected but heat capacity includes vibrational modes at these temperatures. The Vulcan results (blue and magenta) are computed for a perfect gas on the very fine grid. They show a larger over-prediction in the extent of separation for this model as compared to LAURA or DPLR. The Vulcan results were executed with $r_{\text {prod,lim }}=5$, more restrictive than LAURA with $r_{\text {prod,lim }}=20$, because unrealistically large values of $k$ and consequently $\mu_{t} / \mu$ are produced in this simulation behind the compression shock if $r_{\text {prod,lim }}>5$. Vulcan was rerun without any production limiting. This change did reduce the extent of separation though it still was larger than indicated by the experimental data and the other simulations. A more comprehensive investigation into the causes of these differences has not yet been accomplished. Note that uncertainty metrics for Vulcan runs using other models on the same grid are captured in Table 3. DPLR results on the baseline grid for pressure are shown in brown. The separation for the baseline SST model in this case is slightly larger than indicated by experiment. The LAURA implementation looks good in this case but it does suffer from unrealistically large values of $k$ behind the compression shock.

These differences suggest a new verification test is needed to confirm that model implementation across codes is consistent. The method of manufactured solutions could fulfill this need provided that the manufactured solution was sufficiently complex as to engage all elements of the model (production limiters, realizability conditions, etc.). Indeed, the flat plate tests in Appendix B did not reveal significant differences in models that were exposed by this wedge problem with separation. A better approach may be to use the restart solutions for a complex flow on two successively refined grids, with and without turbulence models engaged, as the basis of a "manufactured solution". The ability to: (1) transfer the restart solutions for a complex simulation from the converged solution of one code to another; and (2) follow with a contour plot of residuals for every equation prior to any solution update could serve to expose model inconsistencies between codes without the need to run the second code to convergence.

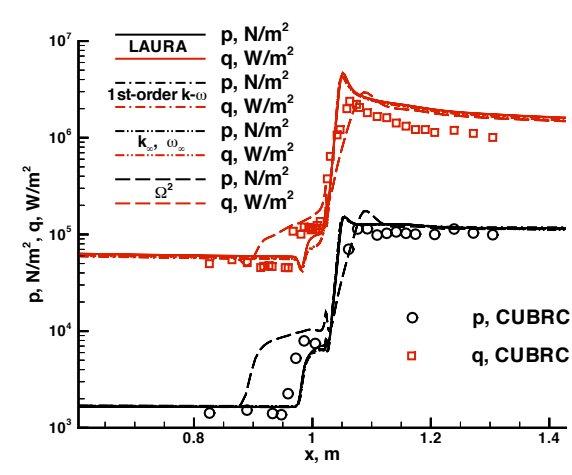

(a) Progression of Models in LAURA

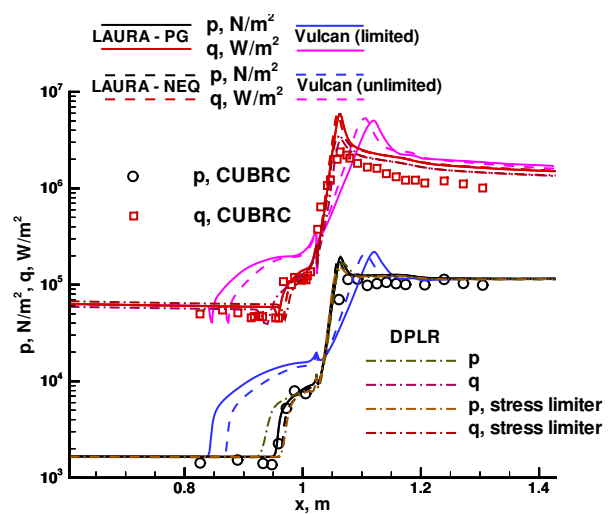

(b) LAURA, Vulcan, DPLR

Figure 8. A progression of turbulence model changes and code implementations for the Menter SST model applied to Run 54 . 


\section{B. CUBRC - Sharp Cone Flare}

Experimental data for a turbulent boundary layer at Mach 11 on a 6 deg. half-angle sharp cone followed by a $36 \mathrm{deg}$. compression (42 deg. half-angle flare) obtained at the 96 inch CALSPAN shock tunnel ${ }^{33}$ are used as the second validation check for the suite of turbulence models applied to shock wave turbulent boundary layer interactions at compression corners. The simulated configuration is presented in Fig. 9. The simulation put a 0.01 inch bluntness on the nosetip. Distances in Fig. 9 are measured from the sphere-cone junction point. (Note that gauge locations are recorded ${ }^{33}$ as distance from the virtual cone vertex and so appropriate transformations are required to compare simulation and experiment.) The baseline grid uses 130 cells along the cone and 128 cells across the shock layer. The near wall region prior to separation is resolved with $y^{+} \approx .29$, achieved by specifying a cell Reynolds number equal to 0.1 in the initial grid generation process. Inflow boundary conditions are: $V_{\infty}=1,807 \mathrm{~m} / \mathrm{s}, \rho_{\infty}=0.032354 \mathrm{~kg} / \mathrm{m}^{3}, T_{\infty}=67.4 \mathrm{~K}$ and $\alpha=0$ deg.. Surface boundary conditions are no-slip with $T_{w}=300 \mathrm{~K}$. Supersonic outflow boundary conditions are extrapolated from the interior. The test gas, air, is treated as calorically perfect. Molecular transport properties are computed from Sutherlands law.

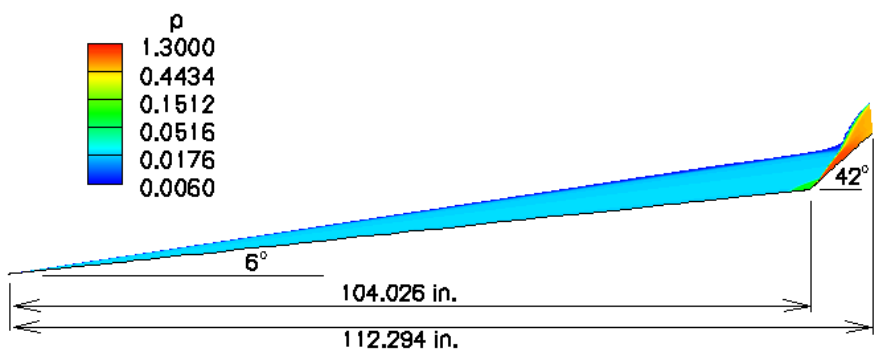

Figure 9. Cone-flare geometry with shock layer pressures non-dimensionalized by $\rho_{\infty} V_{\infty}^{2}$.

Pressure and heating distributions for this case using the algebraic turbulence models are presented in Fig. 10. The Cebeci-Smith model is in good agreement with extent of separation on the baseline grid (Fig. 10(a,b)) but significantly over-predicts separation on the next finest grid (Fig. 10(c,d)) which has a factor of two increase resolution in all directions. The Baldwin-Lomax model under-predicts the extent of separation on both the baseline grid (Fig. 10(e)) and the fine grid (Fig. 10(f)). Good agreement is obtained with pressure and heating levels upstream of the interaction and pressure downstream of the interaction using the Baldwin-Lomax model. Heating levels are under-predicted by approximately $50 \%$ downstream of the interaction. The coarse grained metrics from Eqs. 43 - 48 for this figure are captured in Table 4.

Simulations using the Spalart-Allmaras, Menter SST, and $k-\omega$ (2006) models are compared to experiment in Fig. 11. The Spalart-Allmaras model provides best agreement on the baseline grid with the experimental data. It has not been tested on the next finer grid. Based on previous grid refinement studies it is expected that refinement will cause the extent of separation to further increase. The Menter SST and $k-\omega$ models without compressibility correction show very little separation on the baseline grid. A compressibility correction to the $k-\omega$ (2006) induces separation on the baseline grid. The coarse grained metrics from Eqs. 43-48 for this figure are captured in Table 5 .

Refinement studies continue, focusing on the $k-\omega$ (2006) models in Fig. 12. Results for $M_{t 0}=0.5$ are presented for the coarse and fine grids in Fig. 12 (a,c), respectively. Results for the slightly larger argument to the Heaviside function $\left(M_{t 0}=0.7\right)$ are presented in Fig. $12(\mathrm{~b}, \mathrm{~d})$, respectively. Recall that $M_{t 0}=0.7$ gave the best comparisons to the previous test case (Fig. $6(\mathrm{~b}, \mathrm{f})$ ). In this case, the simulation with $M_{t 0}=0.7$ fails to separate even on the fine grid. (A model without compressibility correction is equivalent to engaging the compressibility correction with a value of $M_{t 0}$ that exceeds the turbulence Mach number everywhere in the flow. Consequently, the simulation without compressibility corrections fails to separate on the fine grid as well.) Best agreement in this case on the fine grid occurs with $M_{t 0}=0.5$. Pressure levels across the recirculation region are well predicted. Both the pressure and heating show an overshoot of $50 \%$ and $80 \%$ respectively after reattachment but then recover the measured levels on the flare. (A drop-off in agreement beyond $\mathrm{x}=109$ is not understood at this time but may be a misunderstanding of the gauge location definition on the flare.) The coarse-grained metrics from Eqs. 43 - 48 for Fig. 6 are captured in Table 6 . 


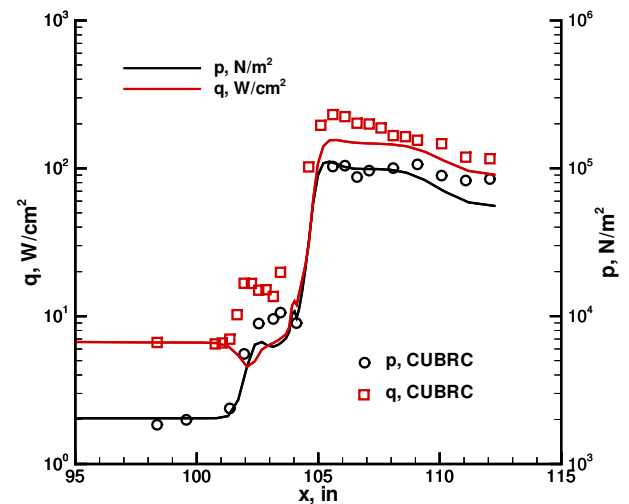

(a) Cebeci-Smith, Eq. 12, (130 x 128)

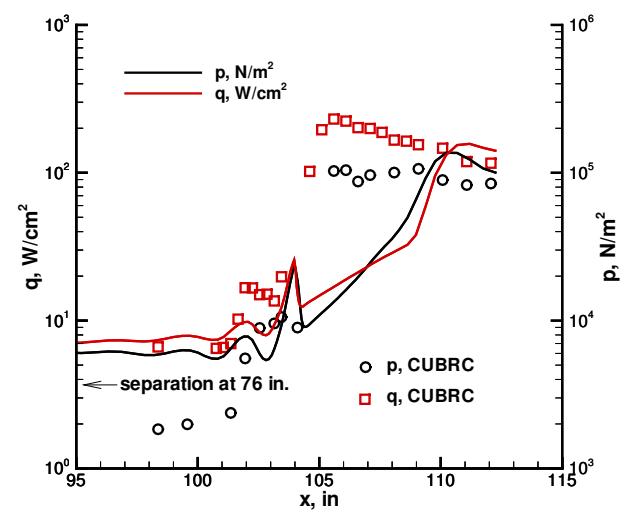

(c) Cebeci-Smith, Eq. 12, (260 x 256)

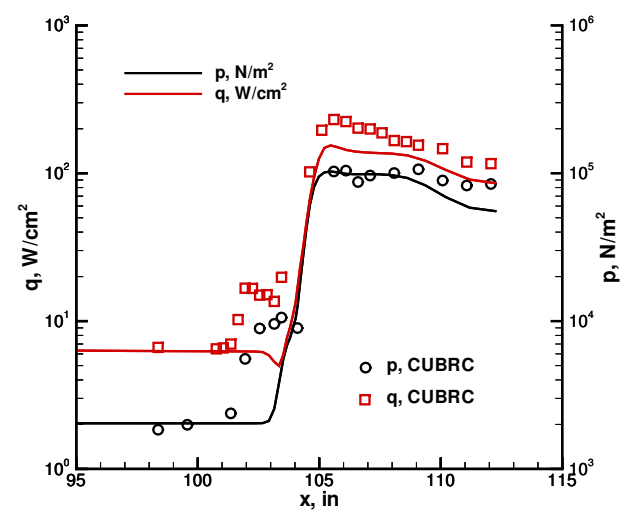

(e) Baldwin-Lomax, (130 x 128)

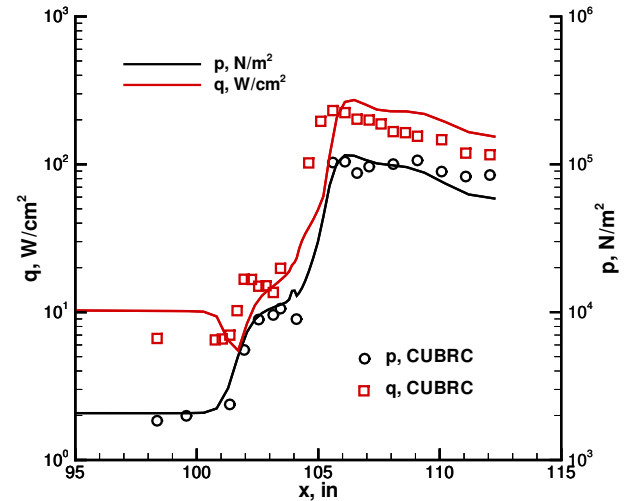

(b) Cebeci-Smith, Eq. 10, (130 x 128)

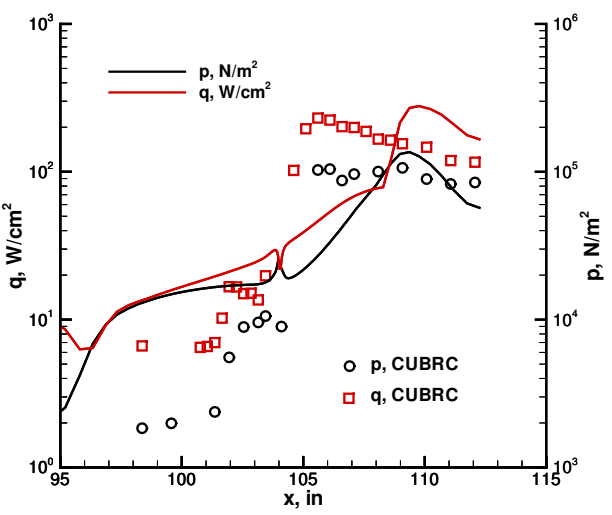

(d) Cebeci-Smith, Eq. 10, (260 x 256)

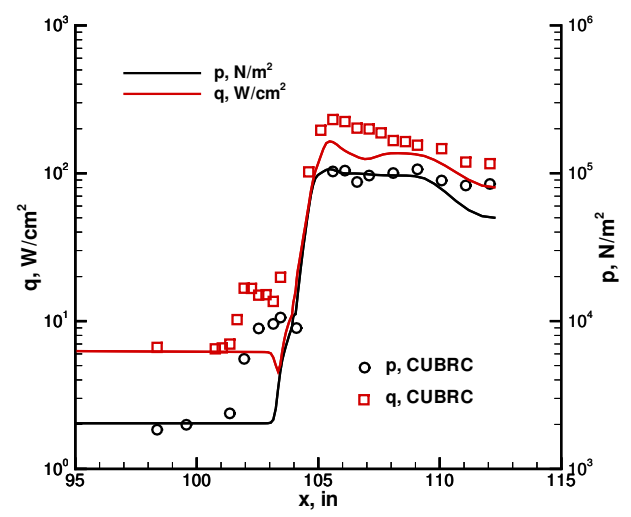

(f) Baldwin-Lomax, (260 x 256)

Figure 10. Pressure and heating distributions over a 6-degree cone with 36-degree compression at Mach 11 computed with algebraic turbulence models. 
Table 4. Coarse-Grained Uncertainty Metrics for Fig. 10

\begin{tabular}{cccccccc}
\hline Model & $E\left(q_{\max }\right)$ & $E\left(p_{\max }\right)$ & $E\left(q_{\text {sep }}\right)$ & $E\left(p_{\text {sep }}\right)$ & $E\left(q_{\text {pre }}\right)$ & $E\left(p_{\text {pre }}\right)$ & $E(L)$ \\
\hline CS $(130 \times 128)$ & -60 & 5 & -130 & -70 & 0 & -5 & 0 \\
CSp $(130 \times 128)$ & 20 & 15 & -10 & 10 & 60 & -5 & -5 \\
CS $(260 \times 256)$ & -60 & 40 & -90 & -80 & NA & NA & NA \\
CSp $(260 \times 256)$ & 30 & 40 & 70 & 90 & NA & NA & -350 \\
BL $(130 \times 128)$ & -60 & 0 & -220 & -130 & -5 & 0 & 95 \\
BL $(260 \times 256)$ & -60 & 0 & -220 & -130 & -5 & 0 & 95 \\
\hline
\end{tabular}

Table 5. Coarse-Grained Uncertainty Metrics for Fig. 11

\begin{tabular}{cccccccc}
\hline Model & $E\left(q_{\max }\right)$ & $E\left(p_{\max }\right)$ & $E\left(q_{\text {sep }}\right)$ & $E\left(p_{\text {sep }}\right)$ & $E\left(q_{\text {pre }}\right)$ & $E\left(p_{\text {pre }}\right)$ & $E(L)$ \\
\hline SA (130 x 128) & -70 & 0 & -80 & -200 & 20 & -5 & 70 \\
SST (130 x 128) & 160 & 0 & -240 & -100 & 20 & -5 & 100 \\
$k-\omega 06(130 \times 128)$ & 5 & -5 & -240 & -110 & 5 & -5 & 100 \\
$k-\omega 06.25(130 \times 128)$ & 5 & 0 & -240 & -90 & -50 & -10 & 40 \\
\hline
\end{tabular}

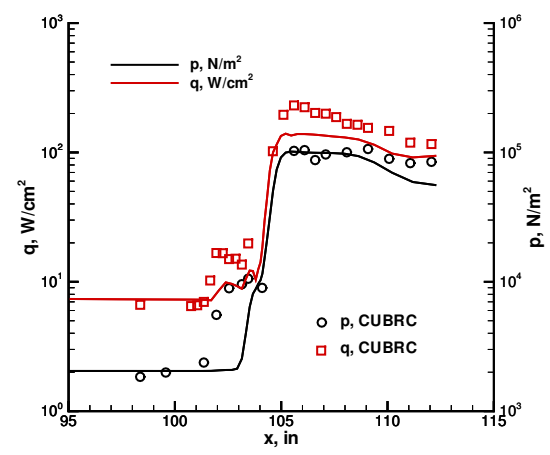

(a) Spalart-Allmaras)

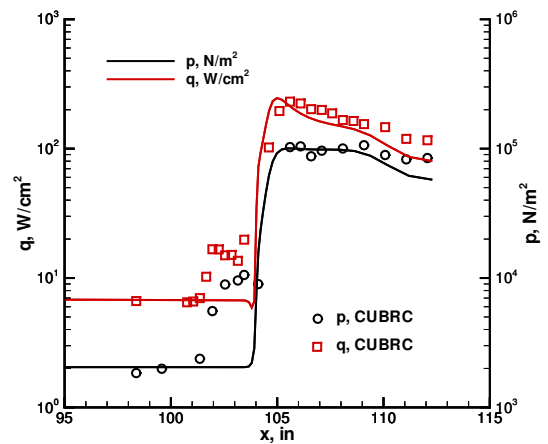

(c) $k-\omega(2006)$, no compressibility correction

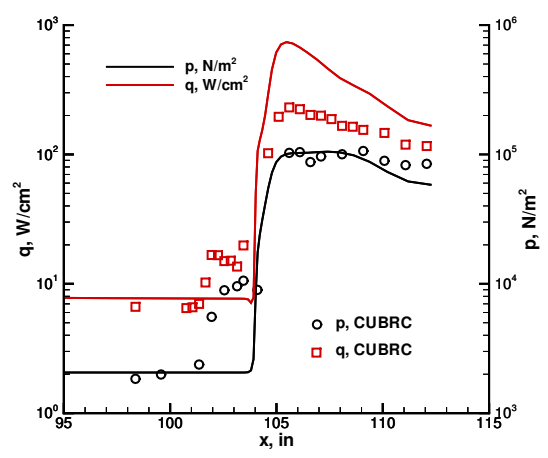

(b) Menter SST

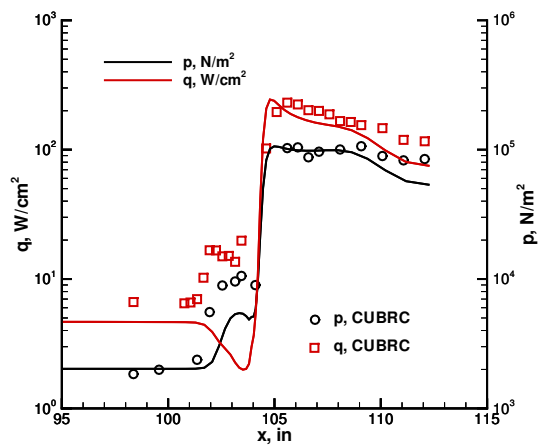

(d) $k-\omega(2006)$, compressibility correction $M_{t 0}=0.25$

Figure 11. Pressure and heating distributions over a 6-degree cone with 36-degree compression at Mach 11 computed with 1- and 2-equation models on baseline grid (130 x 128). 
Table 6. Coarse-Grained Uncertainty Metrics for Fig. 12

\begin{tabular}{cccccccc}
\hline Model & $E\left(q_{\max }\right)$ & $E\left(p_{\max }\right)$ & $E\left(q_{\text {sep }}\right)$ & $E\left(p_{\text {sep }}\right)$ & $E\left(q_{\text {pre }}\right)$ & $E\left(p_{\text {pre }}\right)$ & $E(L)$ \\
\hline$k-\omega 06.5(130 \times 128)$ & 0 & -5 & -240 & -110 & 5 & 5 & 100 \\
$k-\omega 06.7(130 \times 128)$ & 5 & -5 & -240 & -110 & 5 & 5 & 100 \\
$k-\omega 06.5(260 \times 256)$ & 80 & 50 & -100 & -5 & 5 & 5 & -5 \\
$k-\omega 06.7(260 \times 256)$ & -10 & 0 & -240 & -110 & 5 & 5 & 100 \\
\hline
\end{tabular}

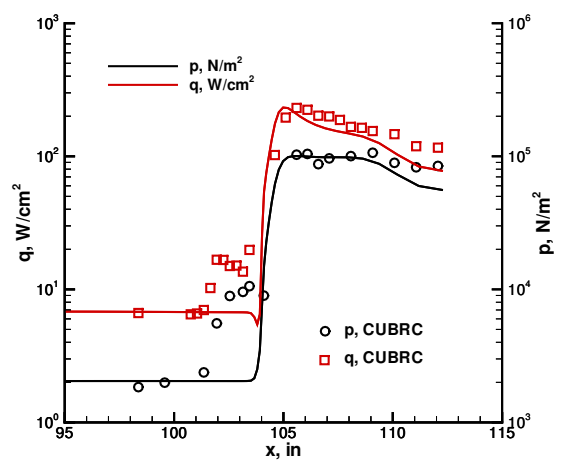

(a) $M_{t 0}=0.50,(130 \times 128)$

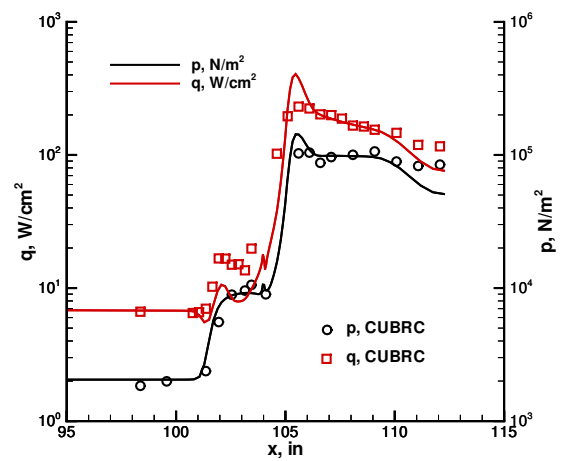

(c) $M_{t 0}=0.50,(260 \times 256)$

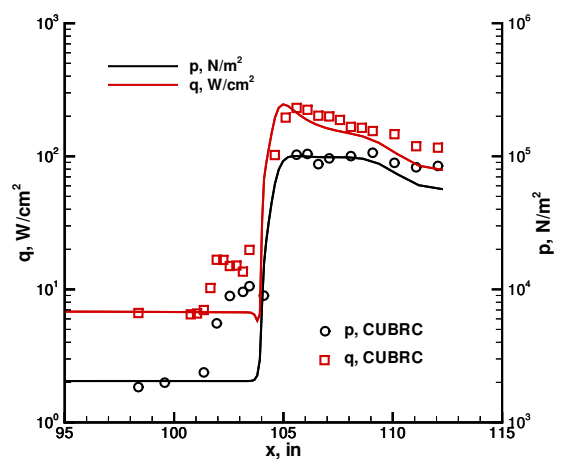

(b) $M_{t 0}=0.70,(130 \times 128)$

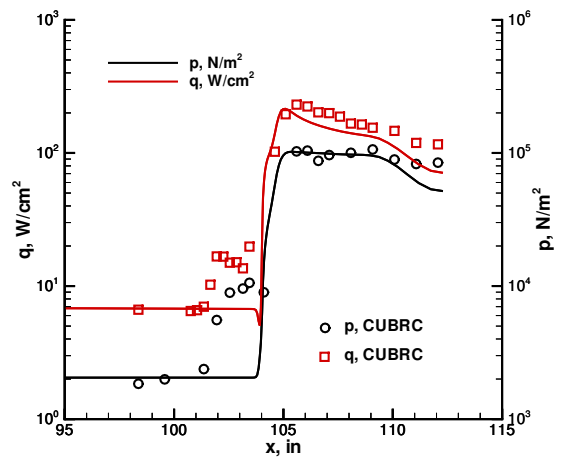

(d) $M_{t 0}=0.70,(260 \times 256)$

Figure 12. Pressure and heating distributions over a 6-degree cone with 36-degree compression at Mach 11 computed with $k-\omega(2006)$ model and non-standard compressibility corrections. 
One additional refinement, another factor of two in each direction $(520 \times 512)$, is applied to the $k-\omega$ (2006) model with $M_{t 0}=0.5$. The results of this simulation on the finest grid are presented in Fig. 13 . There is a small additional movement of the separation point upstream, seen by comparing Fig. 13 (a) to Fig. 12 (c). The pressure and heating overshoots are sharper and larger, now 100\% and 80\%, respectively. Otherwise, the agreement between the fine and very fine grid simulations is good. There is an extremely large change in the temperature and velocity profiles going from the baseline (dashed) to the fine (solid) grids in Fig. 13 (b). Very small changes are evident in these profiles in going from the fine (solid) to finest (dash-dot) grids within the recirculation zone. The near wall region and the inviscid region above the recirculation zone show grid convergence on all three grids. The coarse grained metrics from Eqs. 43 - 48 for Fig. 13 are captured in Table 7.

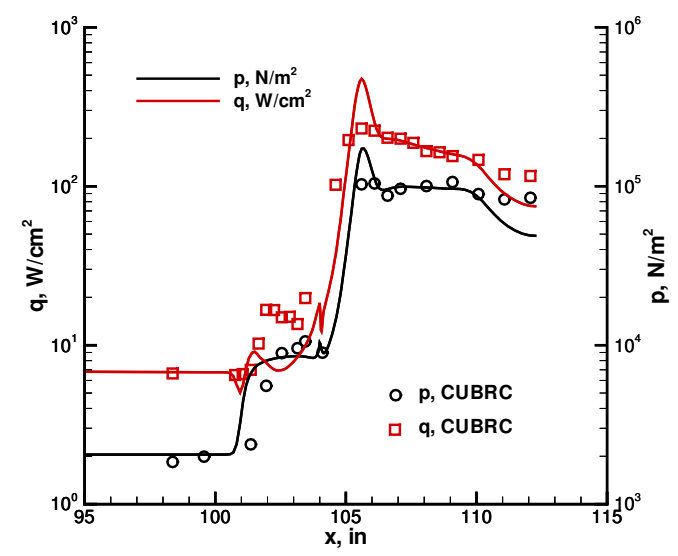

(a) surface pressure and heating, (520 x 512)

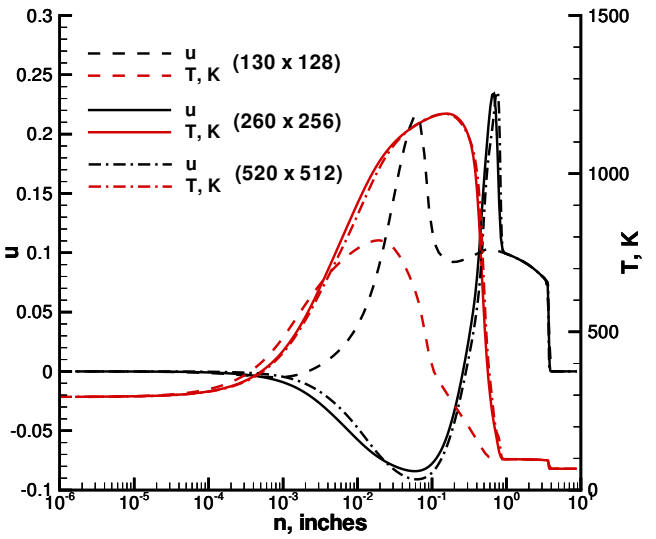

(b) velocity and temperature profiles at cone-flare junction

Figure 13. Simulation results on finest grid (520 x 512) over a 6-degree cone with 36-degree compression at Mach 11 computed with $k-\omega\left(\right.$ 2006) model and $M_{t 0}=0.5$.

Table 7. Coarse-Grained Uncertainty Metrics for Fig. 13

\begin{tabular}{cccccccc}
\hline Model & $E\left(q_{\max }\right)$ & $E\left(p_{\max }\right)$ & $E\left(q_{\text {sep }}\right)$ & $E\left(p_{\text {sep }}\right)$ & $E\left(q_{\text {pre }}\right)$ & $E\left(p_{\text {pre }}\right)$ & $E(L)$ \\
\hline$k-\omega 06.5(520 \times 512)$ & 100 & 80 & -90 & -20 & 5 & 5 & -15 \\
\hline
\end{tabular}

\section{Imperial College no. 2 Gun Tunnel, Nitrogen}

Surface pressure ${ }^{38}$ and heating ${ }^{39}$ measurements for nominal Mach 9.22 flow over a 2D wedge with compression angles from 15 to 38 degrees have been used by several authors (see review by Roy and Blottner ${ }^{12}$ ) for code validation. We capture simulation metrics for several implementations of the $k-\Omega$ model by Huang and Coakley $^{40}$ (34 degree ramp) and Coratekin et al. ${ }^{41}$ (38 degree ramp) in Table 8. The "res?" notation in the table indicates it is not clear that solutions are grid converged in the streamwise direction based on grid requirements for the CUBRC wedge tests. The "NA" notation indicates the metric is not available because it could not be read from the figures or tables.

\section{Rodi Model Simulations}

A variation of the $k-\epsilon$ model by Rodi ${ }^{42}$ and tested by Horstman ${ }^{43}$ appeared to be one of the better models considered in the review by Roy and Blottner. ${ }^{12}$ We have not tested the model ourselves but want to cast a wide net for the purpose of assessing simulation uncertainty for the SWTBLI at compression corners. 
Table 8. Coarse-Grained Metrics for Coleman-Stollery Wedge

\begin{tabular}{ccccccccc}
\hline Description & Model & $E\left(q_{\max }\right)$ & $E\left(p_{\max }\right)$ & $E\left(q_{\text {sep }}\right)$ & $E\left(p_{\text {sep }}\right)$ & $E\left(q_{\text {pre }}\right)$ & $E\left(p_{\text {pre }}\right)$ & $E(L)$ \\
\hline H\&C & $k-\omega 88$ res? & 80 & -10 & NA & NA & NA & NA & 100 \\
H\&C & $k-\omega 88$ cor res? & 0 & 2 & NA & 0 & NA & NA & -60 \\
C\&vK\&B & $k-\omega 88$ res? & 100 & -20 & 90 & -50 & NA & NA & 40 \\
C\&vK\&B & $k-\omega 88$ LS res? & 90 & -20 & NA & NA & NA & NA & $40 ?$ \\
C\&vK\&B & $k-\omega 88$ RC res? & -10 & -25 & 150 & 20 & NA & NA & 20 \\
\hline
\end{tabular}

Uncertainty metrics are recorded in Table 9 for simulations of a 2D compression corner (Mach 9.2), ${ }^{38,39}$ a cone flare (Mach 9.2), ${ }^{33}$ and the cylinder-flare configuration of Kussoy and Horstman (Mach 7.1) ${ }^{44}$ and Coleman (Mach 9.2) ${ }^{45}$ Grid convergence tests were reported for these simulations using grids of 80 to 150 points in the $x$-direction and 36 to 60 points in the $y$-direction. These grid resources are significantly less than those required in LAURA (see, for example, Fig. 13) for the same simulations to achieve a grid-converged solution.

Table 9. Coarse-Grained Uncertainty Metrics for Rodi Model

\begin{tabular}{ccccccccc}
\hline Description & Mach & $E\left(q_{\max }\right)$ & $E\left(p_{\max }\right)$ & $E\left(q_{\text {sep }}\right)$ & $E\left(p_{\text {sep }}\right)$ & $E\left(q_{\text {pre }}\right)$ & $E\left(p_{\text {pre }}\right)$ & $E(L)$ \\
\hline cyl - 20 deg. flare & 7.1 & 20 & 5 & 0 & 0 & NA & NA & none \\
cyl - 35 deg. flare & 7.1 & 20 & 10 & -40 & $20 ?$ & NA & NA & 0 \\
2D cc - 34 deg. & 9.2 & 35 & -10 & NA & NA & NA & NA & 25 \\
cyl - 40 deg. flare & 9.2 & 35 & -20 & NA & NA & NA & NA & 0 \\
cone - 36 deg. flare & 11. & -20 & -10 & NA & -30 & NA & NA & 10 \\
\hline
\end{tabular}

\section{E. CUBRC Compression Corner - Runs 12, 16, and 19}

Free stream conditions for three addition CUBRC Compression corner simulations are provided in Table 10. The wedge geometries have a running length of $39.2 \mathrm{in}(.99568 \mathrm{~m})$ to the corner. The simulations were run with grids of 460 cells in the streamwise direction and 128 cells in the normal direction. Grid metrics are believed to be equivalent to the fine grid used in Run 54 simulations. Runs 12 and 16 show no separation at the corner and the associated simulations also indicate attached flow. A reduced set of uncertainty metrics for these first two cases are presented in Table 11. The experimental data for Run 19 shows separated flow but none of the simulations computed any separation. The metrics for this case are captured in Table 12.

Table 10. Free stream conditions for CUBRC Runs 12, 16, and 19

\begin{tabular}{cccccc}
\hline Run & wedge angle, deg. & $\rho_{\infty}, \mathrm{kg} / \mathrm{m}^{3}$ & $V_{\infty}, \mathrm{m} / \mathrm{s}$ & $T_{\infty}, \mathrm{K}$ & $M_{\infty}$ \\
\hline 12 & 27 & 0.5090 & 1390. & 71.1 & 8.2 \\
16 & 30 & 0.5060 & 1375. & 68.9 & 8.3 \\
19 & 33 & 0.4910 & 1379. & 71.7 & 8.1 \\
\hline
\end{tabular}


Table 11. Coarse-Grained Uncertainty Metrics for Unseparated Flow, CUBRC Runs 12 and 16

\begin{tabular}{ccccc}
\hline & \multicolumn{2}{c}{ Run $12,27^{\circ}$} & \multicolumn{2}{c}{ Run 16, 30 } \\
Model & $E\left(q_{\max }\right)$ & $E\left(p_{\max }\right)$ & $E\left(q_{\max }\right)$ & $E\left(p_{\max }\right)$ \\
\hline Menter SST & 75 & 0 & 70 & 5 \\
$k-\omega 98$ & 75 & 0 & 70 & 5 \\
$k-\omega 06$ & 50 & 5 & 45 & 0 \\
\hline
\end{tabular}

Table 12. Coarse-Grained Uncertainty Metrics for CUBRC Run 19

\begin{tabular}{cccccccc}
\hline Model & $E\left(q_{\max }\right)$ & $E\left(p_{\max }\right)$ & $E\left(q_{\text {sep }}\right)$ & $E\left(p_{\text {sep }}\right)$ & $E\left(q_{\text {pre }}\right)$ & $E\left(p_{\text {pre }}\right)$ & $E(L)$ \\
\hline Menter SST & 30 & -25 & -35 & -75 & 15 & 0 & 100 \\
$k-\omega 98$ & 30 & -25 & -35 & -75 & 15 & 0 & 100 \\
$k-\omega 06$ & 15 & -25 & -35 & -75 & 15 & 0 & 100 \\
\hline
\end{tabular}

\section{Uncertainty Estimates}

We define a simple uncertainty metric $E_{C C}(C C$ for compression corner) that tries to capture the diverse set of comparison metrics recorded in Tables $2-12$. We observe that in current models, if the separation extent is well predicted, the post interaction peak values are not well predicted. In like manner, if the post interaction peak values are well predicted, the extent of separation is not well predicted. This behavior is captured with the following averaged metric, using the most conservative approximation that the experimental uncertainties are epistemic - with no knowledge of the probability of where a measurement may exist within the uncertainty interval.

$$
E_{C C}=E_{C C, \text { num }}+E_{C C, \exp }=\frac{1}{3}\left(\left|E\left(q_{\max }\right)\right|+\left|E\left(p_{\max }\right)\right|+|E(L)|\right)+\frac{1}{3}\left(\left|\Delta q_{\text {exp }}\right|+\left|\Delta p_{\text {exp }}\right|+\left|\Delta L_{\text {exp }}\right|\right)
$$

Discretization error is not included here because grid convergence studies indicate discretization error is much smaller than the other identified sources of uncertainty. We note that there are fifty-four simulations included in the tables involving five experimental data sets from CUBRC, one from Imperial College, and one from NASA Ames. There are thirty-six new simulations using LAURA, six new simulations using Vulcan, and one new simulation using DPLR. Ten entries in the tables sample simulations that are previously published. Table 13 reviews maximum, minimum, average, and median values of $E_{C C, \text { num }}$ for various groupings of simulations.

The first row of Table 13 (All) includes metrics for all models on all configurations. The next eight rows of Table 13 include metrics for a specific turbulence model on all configurations where separation was observed experimentally. Of these models, the sixth row $(k-\omega 06-\mathrm{cmp})$ includes compressibility corrections with various values of $M_{t 0}$; other compressibility corrections and other techniques for turning off their influence near the wall have not been tested. The tenth row (unseparated) gathers metrics for simulations of configurations where separation was not observed experimentally. The simulations tend to do a better job for attached flows and so these cases were isolated so as not to skew results for the more challenging separated flow cases. The last row (fine, very fine) only considers simulations that were done on the two finest grids for which discretization error is demonstrably smaller than other sources of error.

In computing a representative metric of uncertainty for production ready models as discussed in the introduction we discount some entries in Table 13. Results from the algebraic models (row 7) are not included because these models failed to converge for several separated flow conditions. Results from earlier simulations in rows 8 and 9 are not included because of concerns that the grid densities used to compute these cases were considerably less than grid densities required here to demonstrate convergence. Continued grid refinement to the fine and very fine levels used herein can actually degrade agreement with experimental data for some 
Table 13. $E_{C C, \text { num }}$ norms

\begin{tabular}{|c|c|c|c|c|c|c|}
\hline Set Description & Number in Set & $E_{C C, \text { num }, \min }$ & $E_{C C, \text { num }, \max }$ & $E_{C C, \text { num }, \text { avg }}$ & $E_{C C, \text { num }, \text { med }}$ & Notes \\
\hline All & 53 & 8.3 & 140.0 & 43.5 & 40.0 & Omit 4.3 \\
\hline $\mathrm{SA}$ & 2 & 20.0 & 46.7 & 33.3 & 33.3 & \\
\hline SST & 7 & 28.3 & 106.7 & 65.2 & 51.7 & Omit unsep. \\
\hline$k-\omega 98$ & 4 & 40.0 & 55.0 & 49.6 & 51.7 & Omit unsep. \\
\hline$k-\omega 06$ & 7 & 33.3 & 48.3 & 42.1 & 43.3 & Omit unsep. \\
\hline$k-\omega 06-\mathrm{cmp}$ & 12 & 15.0 & 86.7 & 49.9 & 41.7 & Sarkar/Zeman \\
\hline Algebraic & 5 & 13.3 & 140.0 & 55.7 & 51.7 & Omit 4.3 \\
\hline$k-\omega 88$ & 5 & 18.3 & 63.3 & 41.1 & 50.0 & Table 8 \\
\hline Rodi & 4 & 10.0 & 23.3 & 16.3 & 15.8 & Omit unsep. \\
\hline unseparated & 7 & 8.3 & 26.7 & 20.5 & 25.0 & various models \\
\hline fine, very fine & 15 & 33.3 & 106.7 & 60.0 & 55.0 & various models \\
\hline
\end{tabular}

models because the post shock peaks sharpen and rise. (See, for example Fig. 4-e versus Fig. 6-d and Fig. 12 (a versus c).) Consequently, the metrics in the last row of Table 13 , specifically $E_{C C, \text { num, med }}=55$, are thought to be the most representative assessment of the numerical component of uncertainty for simulations of SWTBLI at compression corners sufficiently steep to cause separation. In like manner, $E_{C C, \text { num,med }}=25$ from row 10 is considered a reasonable assessment of the numerical component of uncertainty for simulations of SWTBLI at compression corners where flow remains attached. Using experimental uncertainty estimates from Sec. VI - B the total uncertainty for separated flows (or conditions near incipient separation) is

$$
E_{C C}=E_{C C, \text { num }}+E_{C C, \exp }=55+(9.1+5.8+12.6) / 3=64
$$

The corresponding estimate for attached flows, away from an incipient separation domain, is

$$
E_{C C}=E_{C C, \text { num }}+E_{C C, \exp }=25+(9.1+5.8+0) / 3=30
$$

Clearly, this study has not evaluated every permutation of turbulence model and associated corrections to provide the best possible value of $E_{C C}$. Before adding new simulations it is critical that a well defined verification process be established so that consistency of models across codes can be certified. For now, we have defined a process involving a collection of test cases to provide an estimate for the state-of-the-art using standard models. We expect this metric to serve as a reasonable goal to evaluate improvements to the state-of-the-art for compression corner problems. As simulations improve, a more comprehensive, perhaps fine-grained metric, will be required. For example, the current definition of $E_{C C, \text { num }}$ could equal zero and still have significant errors in the plateau region predictions for heating and pressure. This simple metric and target uncertainty serve the original purpose of providing the Fundamental Aeronautics Program with useful parameters and processes for evaluating improvements to the state-of-the-art in hypersonic simulation.

\section{Simulations of Mission Relevant Test Problem}

The mission relevant test problems were defined previously in Sec. II. New simulations are executed to assess additional uncertainties due to high temperature effects. Higher temperatures are progressively encountered as Mach number is increased and as compression angle is increased. The Spalart-Allmaras (SA) model and Menter-SST model have been applied to the test problems with perfect gas and 5-species models for air specifying equilibrium, finite-rate (chemical nonequilibrium), and frozen flow. Representative results follow.

The Mach 7 simulations indicate peak temperature in the boundary layer approaching the interaction is approximately $780 \mathrm{~K}$. The post-compression peak temperature is approximately 1,260 $\mathrm{K}$ (nonequilibrium model) or $1,380 \mathrm{~K}$ (perfect-gas model). There is no significant chemical dissociation at these conditions. Consequently, all of the 5 -species models are expected to yield equivalent results because they use identical 
thermodynamic and transport property models. Figure 14 for Mach 7 confirms this expectation. It shows that equilibrium and non-equilibrium results are nearly identical on the scale of the figure for shallow (a) and steep (b) compression angles using two different turbulence models. The perfect-gas model shows a $14 \%$ high heating level compared to the high temperature gas models for the shallow compression with the SA model. The perfect gas pressure distribution is in excellent agreement with the high temperature gas model pressure distributions. At Mach 7 it is evident that the perfect-gas models introduce some error in heating distribution due to use of constant heat capacity and use of Sutherlands law. The augmentation of uncertainty at Mach 7 due to high temperature gas effects for the mission relevant test problem is therefore negligible relative to the baseline model form uncertainty defined previously assuming an appropriate gas model is used.

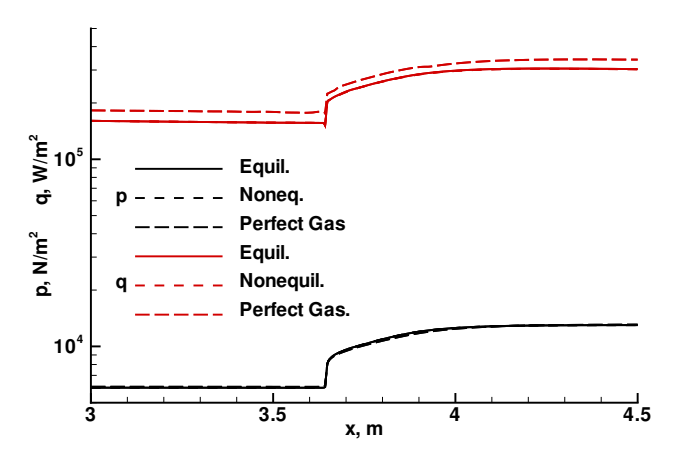

(a) Mach 7,5.5 compression, SA model, (500 x 64)

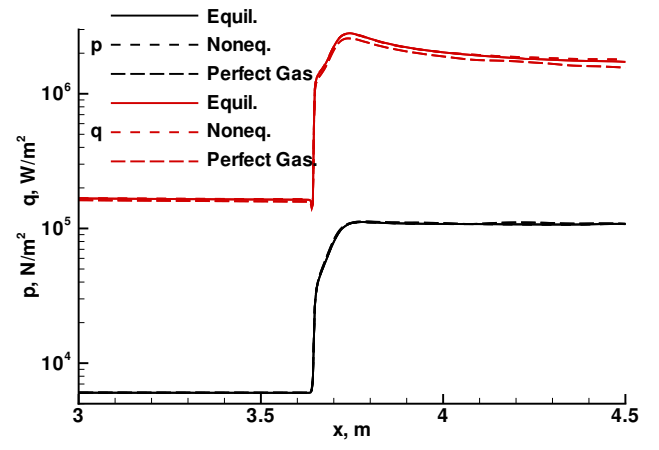

(b) Mach 7, 30 compression, SST model, $(500 \times 64)$

Figure 14. Effects of gas model on simulation of surface pressure (black lines) and heating (red lines) for turbulent flow over test configuration at Mach 7 .

Figure 15 shows shows surface pressure and heating levels across the interaction region of the $5.5^{\circ}$ compression test case using the Menter SST model at Mach 14. The pressure levels approaching the interaction are essentially independent of the 5-species gas model used in the simulation. The non-equilibrium simulation shows a $15 \%$ higher heating than the frozen or equilibrium chemical models. The difference here is caused by the use of a fully catalytic boundary condition in all simulations though only the non-equilibrium simulation has a significant catalytic (diffusion) component. In the frozen flow model there is no dissociation so there is no catalytic component to heating. In the equilibrium flow model the temperature in the near wall region is too low to enable any significant level of atoms; so catalytic heating is again suppressed. The non-equilibrium simulations indicate peak temperature in the boundary layer approaching the interaction is approximately $2,270 \mathrm{~K}$ and the oxygen mass fraction is approximately 0.054 , mostly coming from a blunted leading edge upstream as will be discussed subsequently. In this case the atoms diffuse to the surface and a catalytic component to heating is realized.

There is no clearly defined pressure or heating maximum in the post interaction domain. This case is unique in that it was run using an earlier version of the mission relevant problem configuration with a 1 $\mathrm{cm}$ leading edge bluntness. Early tests on this configuration with laminar flow (not shown here) indicated entropy effects from streamlines passing through the curved, leading edge bow shock persist to the interaction and cause a more gradual rise in pressure and heating after the compression. This issue is more pronounced for small compressions and large incoming boundary layers. It is evident to a lesser extent in the earlier Mach 7 result of Fig. 14 (a) even though that simulation had a sharp leading edge.

Progressing now to the $30^{\circ}$ compression at Mach 14 the post-compression temperature in the shear layer behind the bow shock - compression shock interaction is approximately $3,200 \mathrm{~K}$ and significant oxygen dissociation follows in the free shear layer from that crossing. Closer to the surface, the temperature behind the compression shock near the wall approaches $3,600 \mathrm{~K}$ with atomic oxygen mass fraction approaching 0.0016. Note that this atomic oxygen mass fraction is considerably less than noted in the previous case. In the previous example with the blunted leading edge significant levels of atomic oxygen produced at the blunted leading edge convect to the compression corner. In the current case the reservoir of atoms that drives catalytic heating has not been augmented from sources at the sharp leading edge. Because there 
is relatively little dissociation near the wall across the compression corner one might expect insignificant differences between 5-species model simulations using chemically frozen, equilibrium, and non-equilibrium gas models. Indeed, the catalytic heating component is less than $0.05 \%$ of the heating rate just upstream of the interaction and distributions of pressure and shear for all 5-species models differ by less than $5 \%$ (Fig. 16). Only the perfect-gas model, inappropriate for these temperatures, shows significant differences ( $7 \%$ for pressure, $20 \%$ for heating). Uncertainty metrics should not be modified based on use of an inappropriate gas model at these mission relevant conditions.

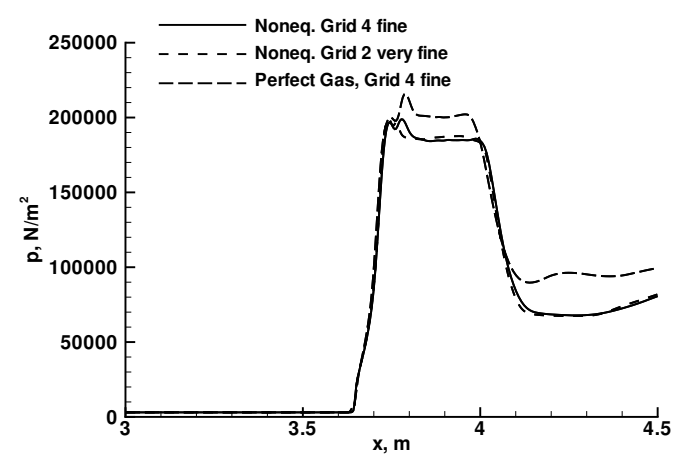

(a) pressure $(512 \times 128)$

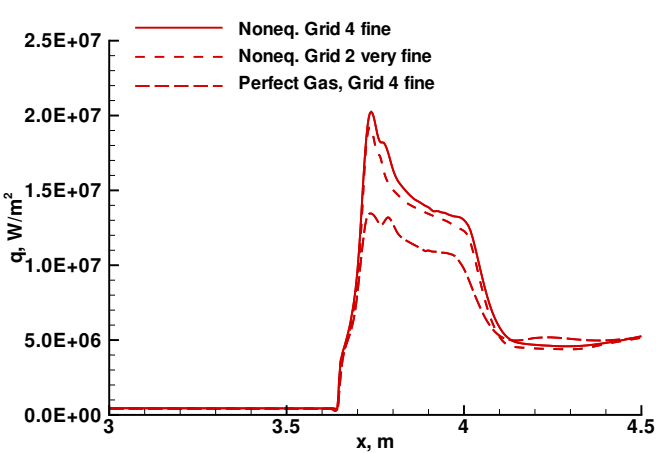

(b) heating $(512 \times 128)$

Figure 15. Effects of gas model on simulation of surface pressure (black lines) and heating (red lines) for turbulent flow over test configuration with $5.5^{\circ}$ compression at Mach 14 using Menter SST model.

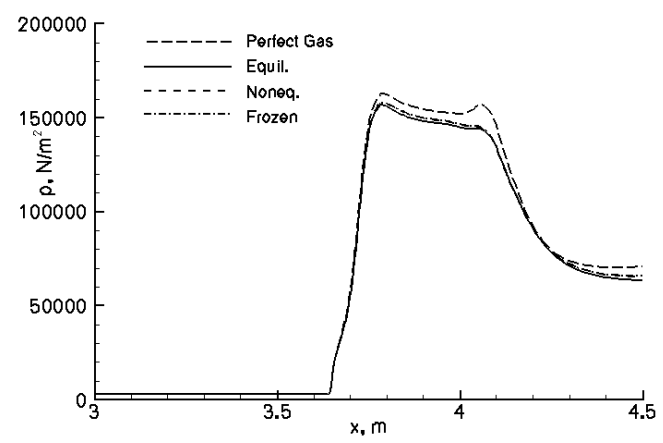

(a) pressure $(500 \times 64)$

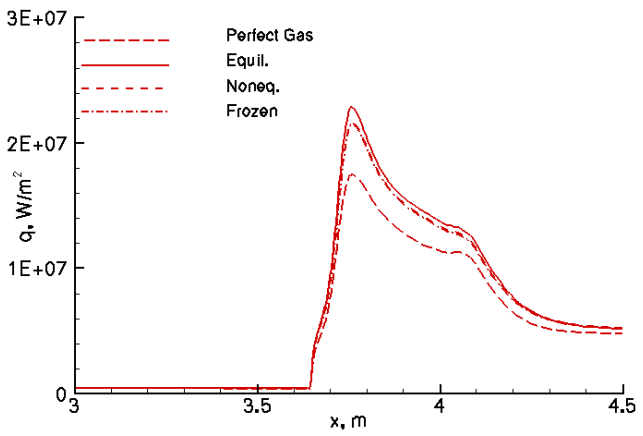

(b) heating $(500 \times 64)$

Figure 16. Effects of gas model on simulation of surface pressure (black lines) and heating (red lines) for turbulent flow over test configuration with $30^{\circ}$ compression at Mach 14.

Simulations of the $5.5^{\circ}$ and $30^{\circ}$ compression corner configurations did not show any separation at Mach 7 or at Mach 14. Simulations of a $35^{\circ}$ compression corner problem using the Menter SST turbulence model with non-equilibrium gas chemistry have been executed on several different grids in the interaction region with focus on resolving an incipient separation. Two of these simulations are considered in Fig. 17. Grid 4 uses the standard grid distribution function and shock alignment algorithms as described earlier for CUBRC Run 54. Grid 2 adjusts the shock alignment algorithm so that the distance between the wall and the inflow boundary downstream of the corner is never allowed to be less than the distance computed by the alignment algorithm at the corner. Grid 4 enables a tighter resolution of the compression shock at the expense of significant grid skewness. Grid 2 has much less grid skewness but the compression shock cuts the grid along diagonals. An overlay of the results using Grid 2 and Grid 4 show good agreement of shock shape and pressure contours. The extent of separation is still small, extending only 2 cells $(\approx 3 \mathrm{~mm})$ upstream from 
the corner on the finest grid.

Corresponding pressure and heating distributions (Fig. 18) show small differences between the peak values (less than $5 \%$ on pressure and less than $8 \%$ on heating) for the same models on two fine grids. The perfect gas results again show more significant differences indicating they are not appropriate for this flow condition.

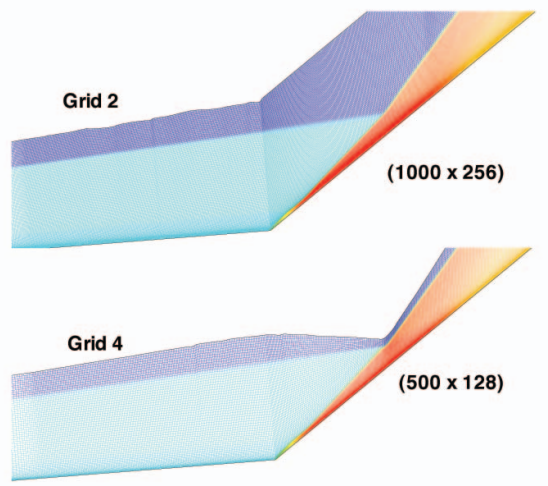

(a) Grid topologies colored by $\log ($ pressure)

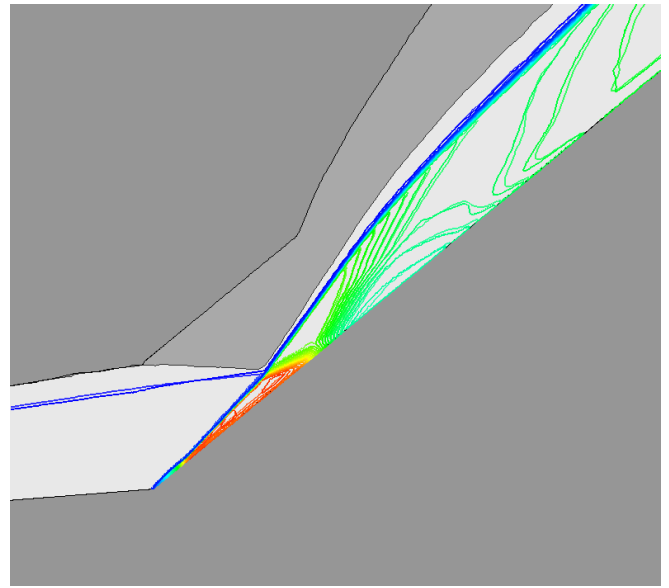

(b) Overlay of pressure contours

Figure 17. Grid topologies used in simulation of test configuration with $35^{\circ}$ compression at Mach 14 .

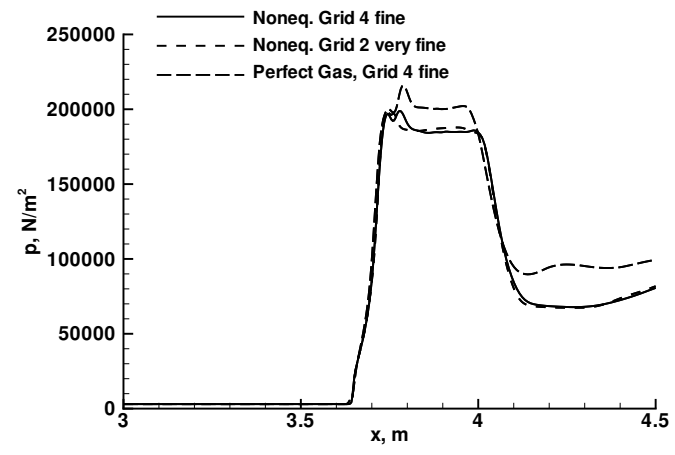

(a) pressure

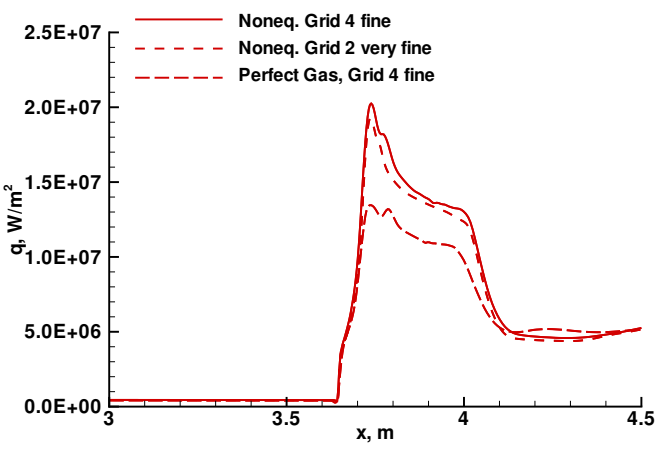

(b) heating

Figure 18. Effects of gas model and grid on simulation of surface pressure (black lines) and heating (red lines) for turbulent flow over test configuration with $35^{\circ}$ compression at Mach 14 using Menter SST model.

The following observations are offered to summarize the impact of chemistry to the uncertainty metrics for mission relevant problems with a sharp leading edge.

- At Mach 7 there is no significant production of atoms and any 5-species air model gives equivalent results.

- At Mach 14 dissociation of atomic oxygen comes in to play, especially for the steeper compressions. The greatest difference in any uncertainty metric as a function of 5 -species model used is less than $5 \%$. This value is considered an upper bound to gas chemistry component of uncertainty.

- Perfect gas models introduce some error at Mach 7 and Mach 14 and are generally not appropriate for mission relevant problem simulations. A perfect gas model shows differences (errors) associated with 
its assumption of constant specific heats at temperatures above 1,000 K encountered at Mach 7 and above.

- Nose bluntness may introduce additional levels of uncertainty associated with chemistry due to the convection of atoms from the nose to the interaction region. The catalytic efficiency of the wall and the distance from the nose to the compression corner become additional factors that impact the uncertainty metrics.

\section{Concluding Remarks}

An uncertainty metric is defined for simulation of a shock wave turbulent boundary layer interaction (SWTBLI) at two-dimensional or axi-symmetric compression corners using production-ready turbulence models. The metric is intended for use by NASA's Fundamental Aero Program to provide a baseline circa 2006 for judging improvements to hypersonic simulation as a result of investments in modeling and experiments.

A mission relevant test problem is defined including free stream conditions for which the simulation uncertainty will be characterized. The governing equations and turbulence models are fully documented herein to remove any ambiguity of how models are formulated. A set of coarse grained uncertainty metrics are introduced to characterize the essential elements of a SWTBLI within a simulation. These metrics are also defined to easily capture their values from figures in the literature. Relevant experimental data on compression corners are reviewed and new simulations are executed with a variety of models and codes representative of the state of the art in 2006. The metrics are calculated for both existing and new simulations and a median value equal to $55 \%$ is computed for SWTBLI with separation on the basis of new, grid converged simulations. It is observed that grid converged solutions may enable sharper peaks in post shock pressure and heating as well as a larger extent of separation that may degrade comparisons with experimental data. Finally, new simulations are executed on the mission relevant problem to include gas chemistry perturbations in order to assess an overall uncertainty metric. Two appendices are also provided to present verification and validation data on a simpler flat plate problem.

Two critical needs are identified if further reductions in the uncertainty metrics are required. The first need, verification of models between codes, is an exceptionally challenging problem for separated SWTBLI simulations. A verification exercise on flat plates between LAURA and DPLR failed to identify an implementation difference in the models which was critical in the SWTBLI simulation. In like manner, comparisons of simulations using Vulcan with LAURA and DPLR show differences which are thought to follow from some subtle difference in model implementation but as yet are not explained. It is suggested that the restart solutions for a complex flow on two successively refined grids from a single code could be used as the basis of "manufactured solutions" for other codes. Plotting the residuals from the second code after a single iteration starting from the "manufactured solution" could expose model inconsistencies between codes without the need to run the second code to convergence. The second need, refinement of experimental error estimates, requires both additional experiments covering relevant energies and Reynolds numbers as well as experiments devoted to better define uncertainties in calibration, free stream conditions, flow non-uniformities (temporal and spatial), and boundary conditions.

\section{Acknowledgements}

The authors thank Matt MacLean of CUBRC for many helpful discussions, for providing digital copies of the experimental data associated with their test program ${ }^{17}$ and for providing DPLR runs on shared grids. The authors thank Robert Baurle of NASA Langley for providing new Vulcan runs on CUBRC Run 54. The NASA Fundamental Aeronautics Program sponsored this work. The larger uncertainty quantification team includes Scott Berry, Deepak Bose, Jim Brown, Peter Gnoffo, Brian Hollis, Chris Johnston, Joe Marvin, and Dinesh Prabhu - all providing valuable insights and recommendations as work progressed on respective mission relevant problems. 


\section{Appendix A: Analytic Approximations}

\section{Van Driest Transform I}

Huang and Coleman ${ }^{46,47}$ investigate the generality of the Van Driest transformation $\mathrm{I}^{48}$ on DNS simulations of compressible, wall bounded flows. They found that the transformation tended to collapse the computed velocity profiles to match the incompressible logarithmic law for cold wall channel cases at Mach numbers of 1.5 and 3. In contrast to DNS simulations the velocity profiles computed with RANS models are derived from these fundamental relations. Consequently, transformed velocity profiles provide no new fundamental insight regarding the physics of turbulent boundary layers. Nevertheless, the transformed velocity profiles for the hypersonic simulations are computed to see if the collapsing trends observed by Huang and Coleman are verified in current models.

Given a computed or measured velocity profile $U^{+}\left(y^{+}\right)$the Van Driest transformed profile $U_{V D}^{+}\left(y^{+}\right)$as presented by Huang and Coleman is given by

$$
U_{V D}^{+}=\frac{1}{R_{V D}}\left\{\sin ^{-1}\left[\frac{R_{V D}\left(U^{+}+H_{V D}\right)}{D_{V D}}\right]-\sin ^{-1}\left[\frac{R_{V D} H_{V D}}{D_{V D}}\right]\right\} .
$$

where

$$
\begin{aligned}
R_{V D} & =M_{\tau} \sqrt{(\gamma-1) P r_{t} / 2} \\
H_{V D} & =B_{q} /\left[(\gamma-1) M_{\tau}^{2}\right] \\
D_{V D} & =\sqrt{1+\left(R_{V D} H_{V D}\right)^{2}}
\end{aligned}
$$

The transformed profiles in the RANS simulations are based on computed values of $\tau_{w}$ and $q_{w}$. Note that $B_{q}$ and therefore $H_{V D}$ are negative quantities for cold walls (energy flux into wall, opposite increasing $y^{+}$ direction).

\section{Van Driest Transform II}

The Kármán-Schoenherr equation, ${ }^{49-51}$ relating skin friction to momentum thickness Reynolds number for an incompressible flow, is given by

$$
\bar{C}_{f}=\left[17.08\left(\log _{10} \bar{R} e_{\theta}\right)^{2}+25.11\left(\log _{10} \bar{R} e_{\theta}\right)+6.012\right]^{-1}
$$

Given a numerical simulation of a compressible flow with computed distribution of $\operatorname{Re}_{\theta}$ a mapping to a corresponding incompressible value $\bar{R} e_{\theta}$ to be used in Eq. 56 may be implemented with

$$
\bar{R} e_{\theta}=F_{\theta} R e_{\theta}
$$

The incompressible skin friction coefficient can then be computed in Eq. 56 and a transform back to the compressible value is implemented with

$$
C_{f}=\bar{C}_{f} / F_{c}
$$

The transformation factors $F_{\theta}$ and $F_{c}$ were derived by van Driest ${ }^{52}$ and are expressed as presented by Hopkins and Inouye ${ }^{51}$ by

$$
F_{\theta}=\mu_{e} / \mu_{w}
$$

and

$$
F_{c}=C_{V D}\left[\sin ^{-1}\left(\alpha_{V D}\right)+\sin ^{-1}\left(\beta_{V D}\right)\right]^{-1}
$$

where

$$
\alpha_{V D}=\frac{2 A_{V D}^{2}-B_{V D}}{\sqrt{4 A_{V D}^{2}+B_{V D}^{2}}}, \quad \beta_{V D}=\frac{B_{V D}}{\sqrt{4 A_{V D}^{2}+B_{V D}^{2}}}
$$

and

$$
A_{V D}=\sqrt{\frac{C_{V D} T_{e}}{T_{w}}}, \quad B_{V D}=\frac{T_{e}+C_{V D} T_{e}-T_{w}}{T_{w}}, \quad C_{V D}=0.9 \frac{\gamma-1}{2} M_{e}^{2}
$$

Comparisons of computed skin friction using turbulence models to the van Driest II transforms are implemented as a supplemental verification check. 


\section{Appendix B: Verification and Validation on Flat Plate at Mach 11}

Experimental data for a turbulent boundary layer at Mach 11.1 on a flat plate obtained at CUBRC ${ }^{37}$ are used as a preliminary verification and validation of the suite of turbulence models. Implementation of turbulence models is fraught with opportunities to err in the programming of the model or the coupling of the model with the conservation equations. A verification check is made by comparing to incompressible profiles from the Van Driest transformations discussed in Appendix A.
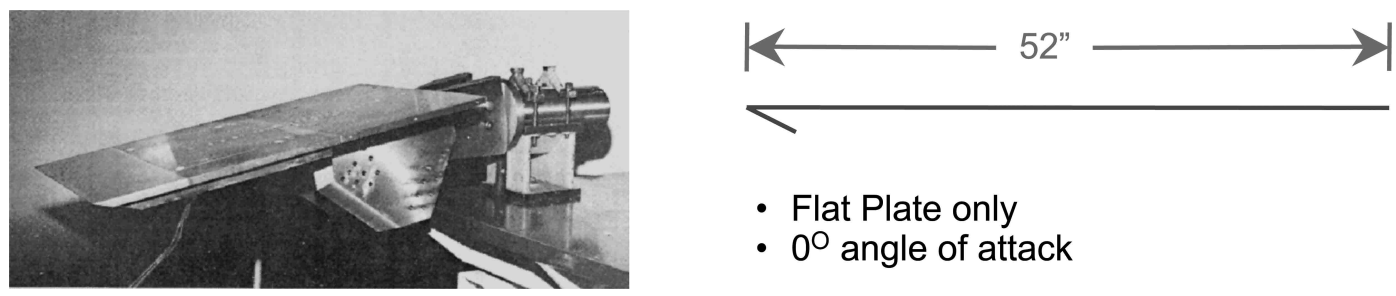

Figure 19. Flat plate geometry for CUBRC Run 7.

The geometry is presented in Fig. 19. The baseline grid uses 300 cells along the plate and 96 cells normal to the plate. The near wall region is resolved with $y^{+} \approx .035$. Inflow boundary conditions are: $V_{\infty}=1,780 \mathrm{~m} / \mathrm{s}, \rho_{\infty}=0.09483 \mathrm{~kg} / \mathrm{m}^{3}, T_{\infty}=64 \mathrm{~K}$ and $\alpha=0 \mathrm{deg}$.. Surface boundary conditions are no-slip with $T_{w}=300 \mathrm{~K}$. Supersonic outflow boundary conditions are extrapolated from the interior. The test gas, air, is treated as calorically perfect. Molecular transport properties are computed from Sutherlands law.

Figure 20 compares experimental data for shear and heating along the surface to simulations using various turbulence models. The solid line result in each figure shows the $k-\omega(2006)$ model distribution - providing a visual reference using the most recent model of the group. The dashed line provides results for the other models. A laminar result is presented in Fig. 20 (a) as a dashed line. The laminar heating result agrees with experimental data for heating prior to transition. The turbulent pressure (black line) exceeds the laminar value due to a larger displacement thickness.

The solid line, $k-\omega(2006)$ without compressibility correction, agrees well with experimental data for shear but over-predicts the turbulent heating rate. (See Sec. V for a discussion of associated experimental uncertainties.) The algebraic models (Fig. 20 (b,c)) show shear levels slightly less than the reference $k-\omega$ (2006) model but still in agreement with the spread in experimental data. The algebraic model heating levels are in better agreement with the experimental data though they still tend to over-predict the heating levels. The 1-eq. Spalart Allmaras model (Fig. 20 (d)) is in good agreement with the $k-\omega(2006)$ model without compressibility correction. There is no pseudo transition point in either the algebraic or 1-eq. models - an increased level of heating and shear exceeding the laminar value persists to the leading edge. The Menter SST model (Fig. 20 (e)) and the older $k-\omega$ (1998) model (Fig. 20 (f)) are bounded by the predictions from the algebraic models and the $k-\omega$ (2006) model. All models are in reasonably good agreement with experimental data for shear and all models exceed the experimental data for heating rate.

The Van Driest II transform (see Appendix A, section B) is used as a verification check of the shear distribution on a flat plate for all models in Fig. 21. The solid red line in these figures is the computed momentum thickness Reynolds number from the simulation. The dashed red line is the transformed incompressible momentum thickness Reynolds number. The solid blue line is the shear distribution calculated from the simulation. The dashed blue line is the Van Driest II shear distribution which is obtained by assuming the Karman-Schoenherr (Eq. 56) can be used to plot the incompressible shear as a function of the transformed momentum thickness Reynolds number and that Eq. 58 can be used to transform the incompressible shear back to a compressible distribution. The shear distribution computed with the $k-\omega$ (2006) model without compressibility correction (Fig. 21 (a)) is in excellent agreement with the Van Driest II transform distribution. The algebraic models (Fig. 21 (b,c)) tend to produce a slightly lower value $\operatorname{Re}_{\theta}$ and a correspondingly higher value of transformed shear. Nevertheless, both values of shear are in good agreement with experimental data. The 1-eq. Spalart-Allmaras model (Fig. 21 (d)) and the 2-eq Menter SST models (Fig. 21 (e)) slightly under-predict the Van Driest II levels but remain in better agreement than either of the algebraic models. The older $k-\omega$ (1998) (Fig. 21 (f)) appears equivalent to the algebraic models in terms of the level of agreement with the transformed shear. 
The Van Driest I transform (see Appendix A, section A) serves as a second verification check by comparing the transformed, incompressible velocity profile to the classical incompressible result with $u^{+}=y^{+}$in the near wall (inner) region and $u^{+}=\ln \left(y^{+}\right) / \kappa+C$ for $y^{+}>10$ to the boundary layer edge (outer). The transformed velocity profiles for each of the turbulence models is presented in Fig. 22 where the compressible velocity profile, non-dimensionalized by $u_{\tau}$ is given by the solid black line, the inner and outer reference profiles with $\kappa=0.41$ and $C=5.0$ are given by the dashed red and blue lines, respectively, and the Van Driest transformed velocity is given by the dash-dot red line. All of the models follow the inner law variation for $y^{+}<3$. The $k-\omega(2006)$ model without compressibility correction (Fig. 22 (a)) and the Menter SST model (Fig. 22 (e)) have transformed velocity profiles that are in excellent agreement with the classical limit in the outer region $\left(y^{+}>10\right)$. The other models remain in fair agreement with the outer limit - small changes in the value of $\kappa$ or $C$ would produce better agreement in these cases. All of the models are in acceptable agreement with the classical limit in this verification check.

The effects of the compressibility correction (see Eq. 29) in the 2-eq. models are explored in Figs. 2325. The compressibility correction is not intended for wall bounded flows. Rather, it is applied to problems involving mixing in free shear layers. The thought here is that compression corners will often induce separated flow and the free shear layer in these cases may not be adequately simulated without some compressibility correction. The concern is that the compressibility correction may significantly distort the results achieved for the fully attached case on the flat plate. Consequently, these corrections are tested here.

Figure 23 follows the same format as Fig. 20. It compares experimental data for shear and heating along the surface to simulations using various turbulence models with a compressibility correction. The solid line result in each figure again shows the $k-\omega$ (2006) model distribution without compressibility correction. The dashed line provides results for the other models now including a compressibility correction. The baseline compressibility correction with $M_{t 0}=0.25$ applied to the $k-\omega$ (2006) model is shown with the dashed lines in Fig. 23 (a). The heating in this case now slightly under-predicts the data and the shear, which had been in good agreement with experiment, now significantly under-predicts the data. As the critical turbulent Mach number is increased (from $M_{t 0}=0.3$ in Fig. 23 (b) to $M_{t 0}=0.5$ in Fig. 23 (d)) the heating and shear distribution approach the compressibility correction free model. A value of $0.3 \leq M_{t 0} \leq 0.4$ slightly compromises the agreement of the baseline model with shear data but improves the agreement with heating data. The compressibility correction behavior for the Menter SST model (Fig. 23 (e)) and the $k-\omega(1998)$ model (Fig. 23 (f)) behave similarly to the $k-\omega(2006)$ model.

The compressibility correction with $M_{t 0} \leq 0.3$ abrogates agreement noted earlier with the Van Driest II transformed shear distribution in Fig. 24 and with the Van Driest I profiles in Fig.25. Only the cases with $M_{t 0}=0.4$ (subfigure (c)) and $M_{t 0}=0.5$ (subfigure (d)) show agreement with the incompressible distributions. Disagreement here is inconsistent with existing DNS simulations at lower edge Mach numbers. 


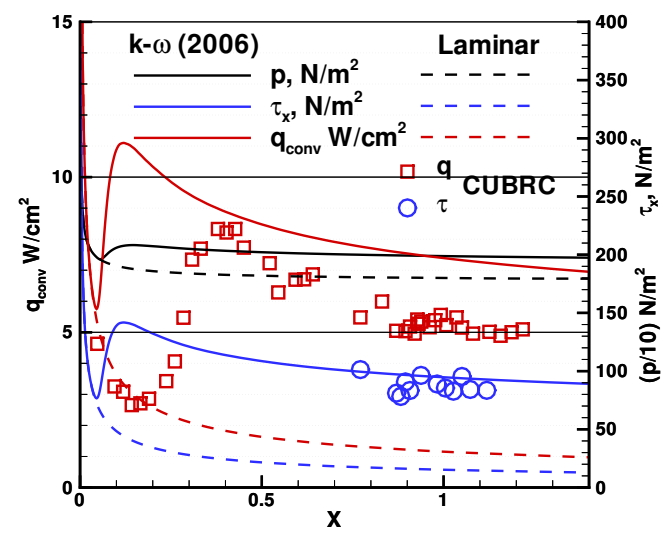

(a) Laminar

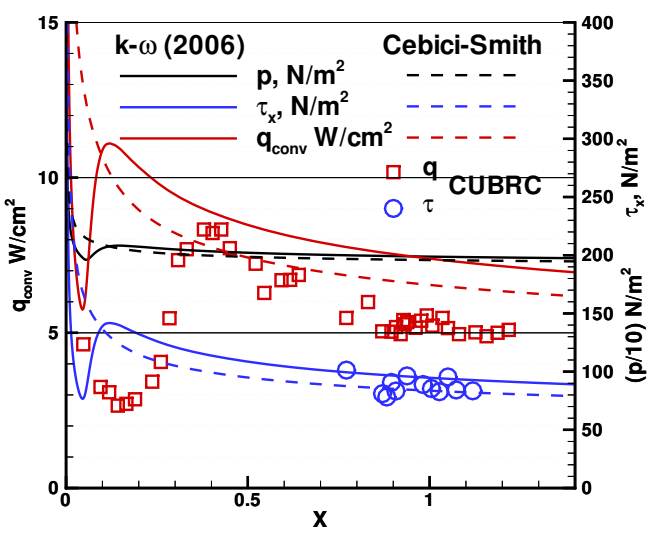

(c) Algebraic: Cebeci-Smith.

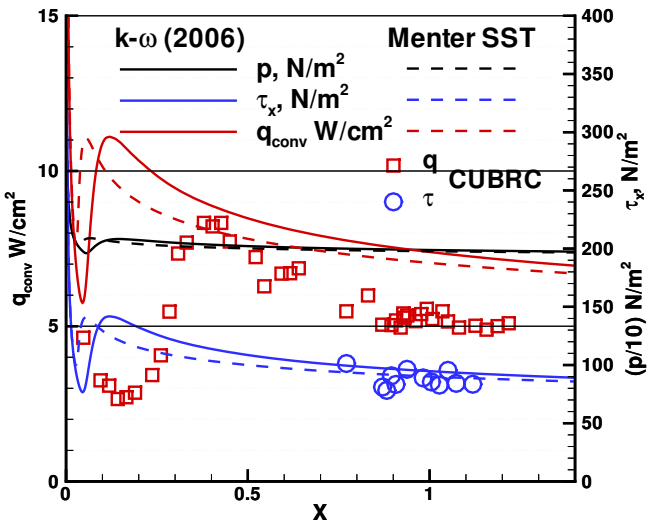

(e) 2-Eq: Menter SST

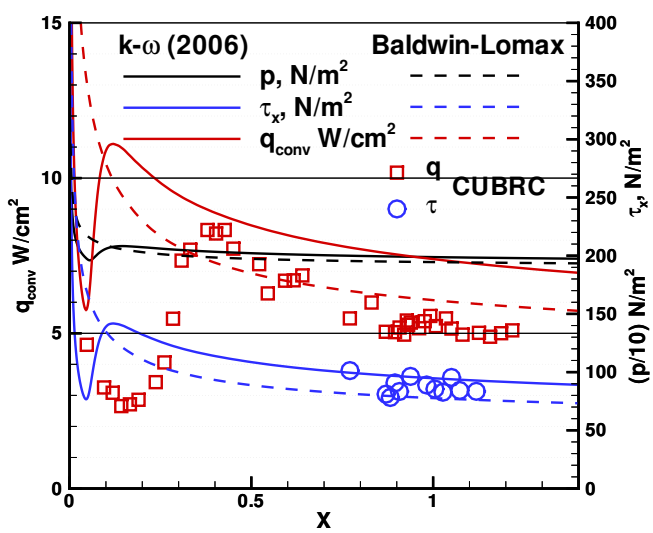

(b) Algebraic: Baldwin-Lomax

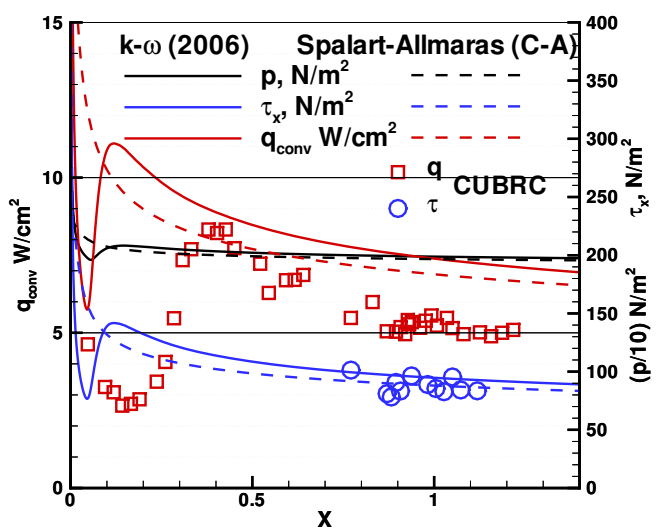

(d) 1-Eq: Spalart-Almaras (Catrix-Aupoix Compressible Model)

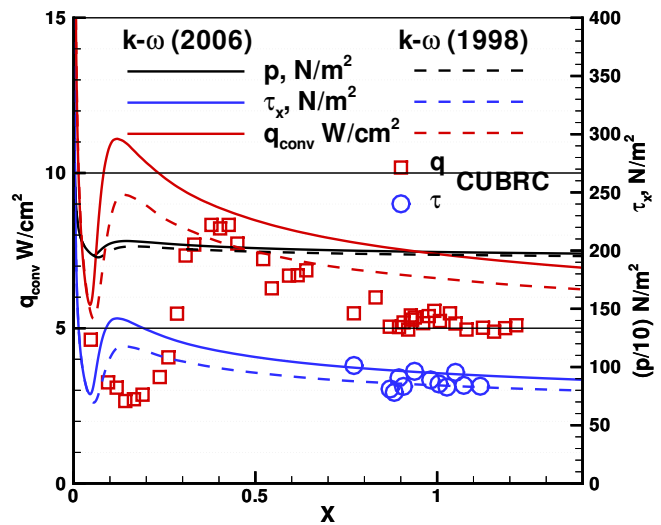

(f) 2-Eq: $k-\omega(1998)$

Figure 20. Pressure, shear, and heating distributions over a flat plate at Mach 11.1 computed with the $k-\omega$ (2006) model compared to experimental data and simulations with other models. 


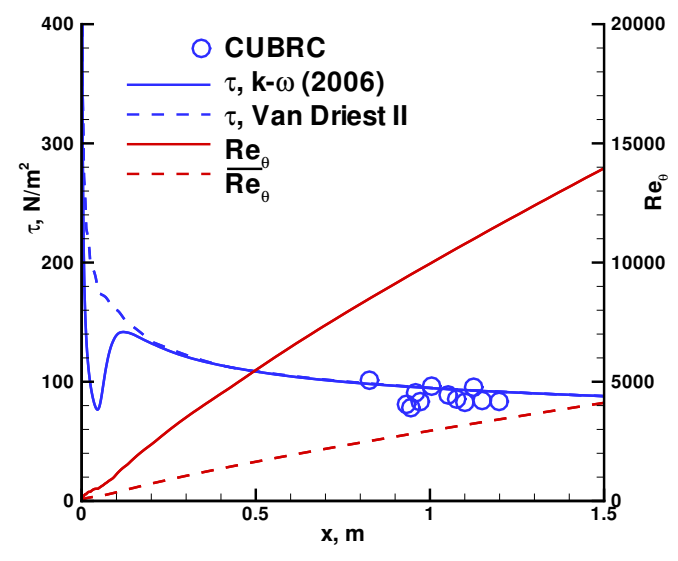

(a) 2-Eq: $k-\omega(2006)$

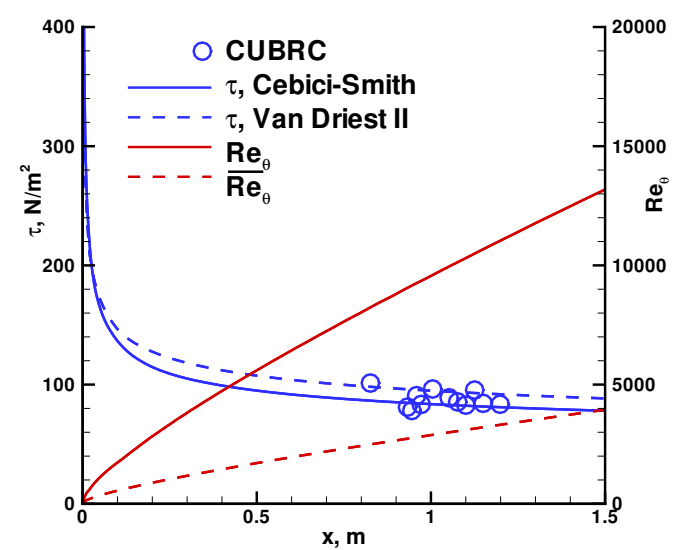

(c) Algebraic: Cebeci-Smith.

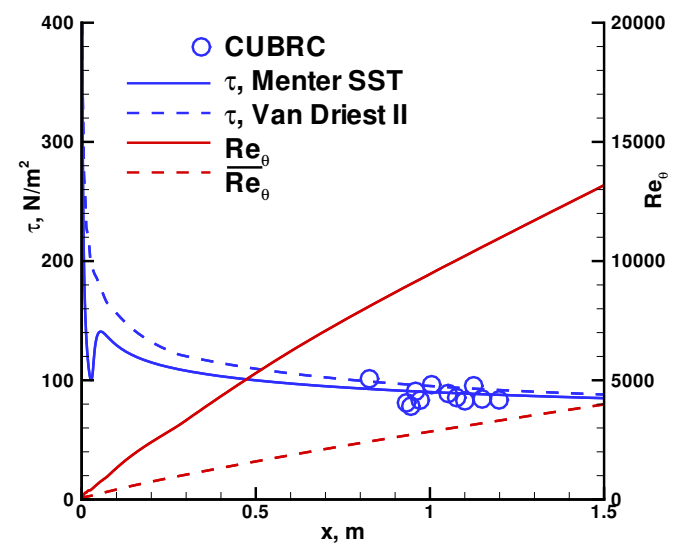

(e) 2-Eq: Menter SST

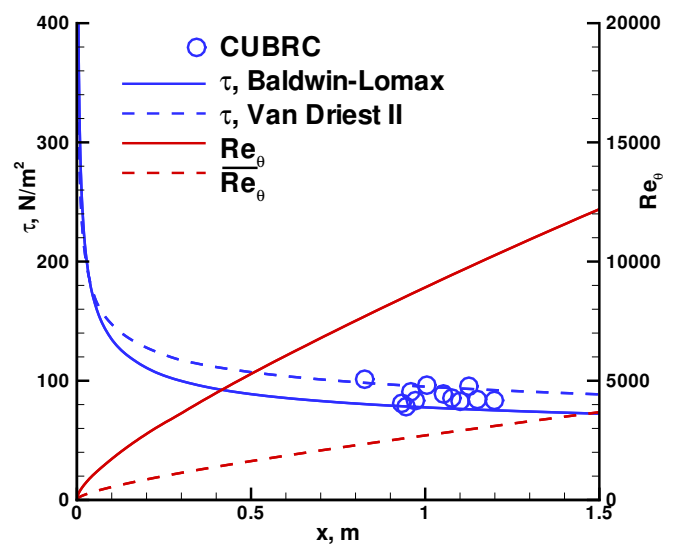

(b) Algebraic: Baldwin-Lomax

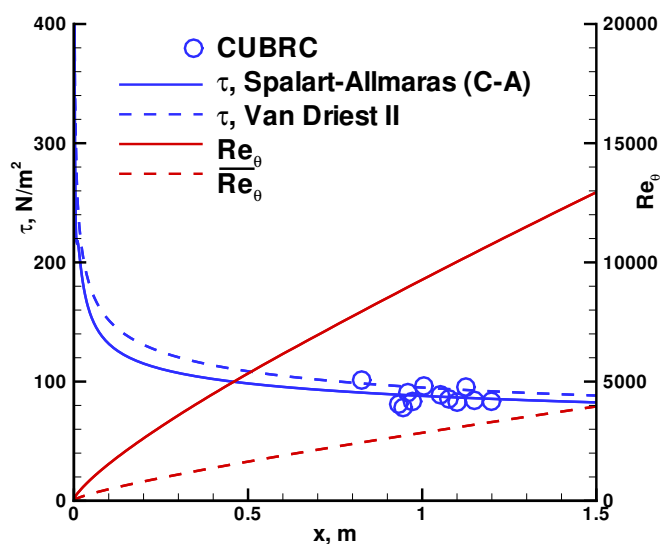

(d) 1-Eq: Spalart-Almaras (Catrix-Aupoix Compressible Model)

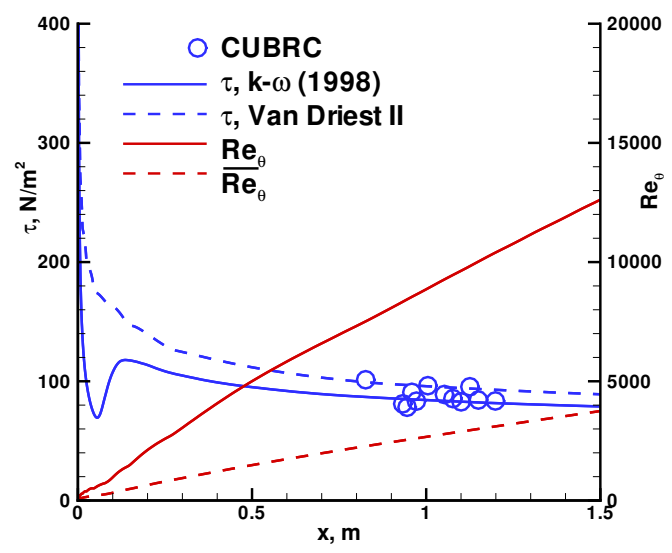

(f) 2-Eq: $k-\omega(1998)$

Figure 21. Shear distribution computed from turbulence models compared to shear computed from Van Driest transformation. The computed momentum thickness Reynolds number distribution from each model and the transformed, incompressible momentum thickness Reynolds number used in the Van Driest transform show consistent magnitudes for all models. 


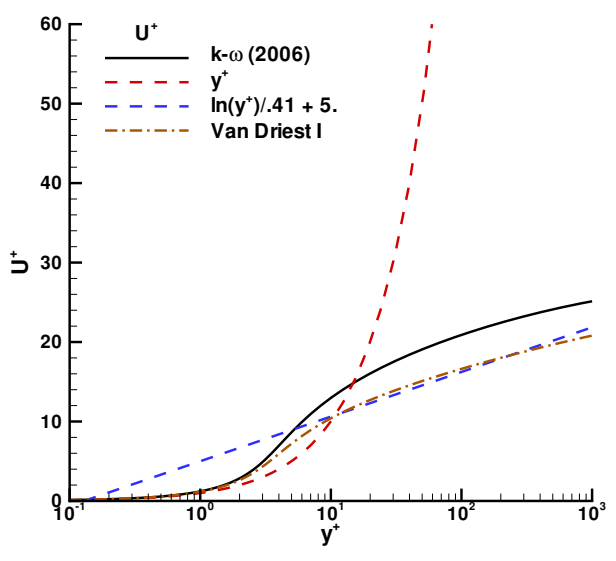

(a) 2-Eq: $k-\omega(2006)$

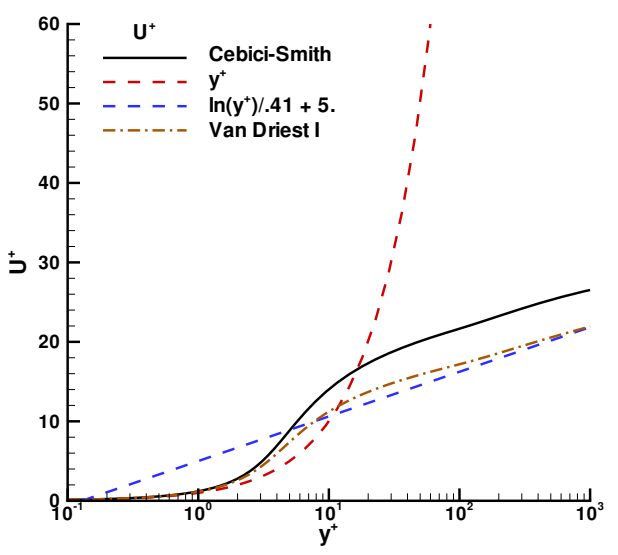

(c) Algebraic: Cebeci-Smith.

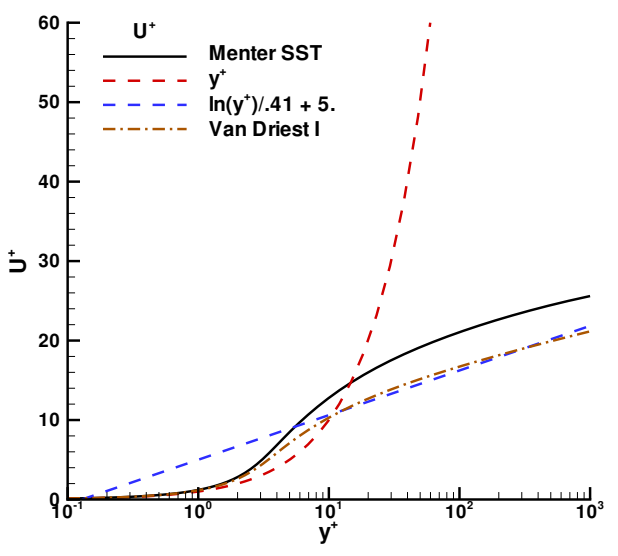

(e) 2-Eq: Menter SST

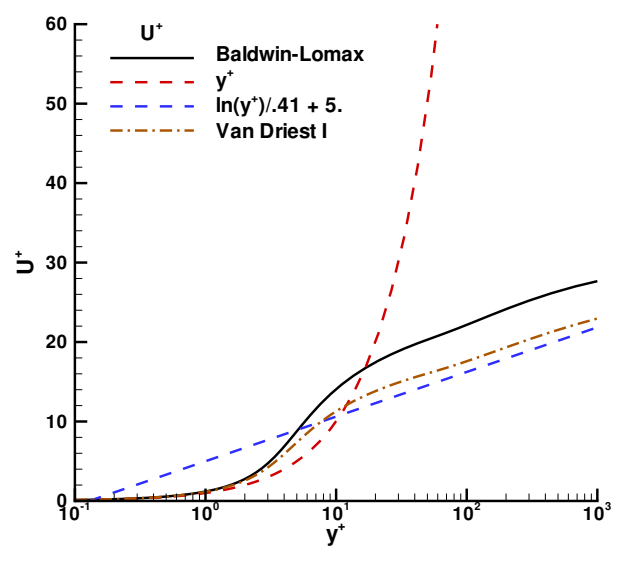

(b) Algebraic: Baldwin-Lomax

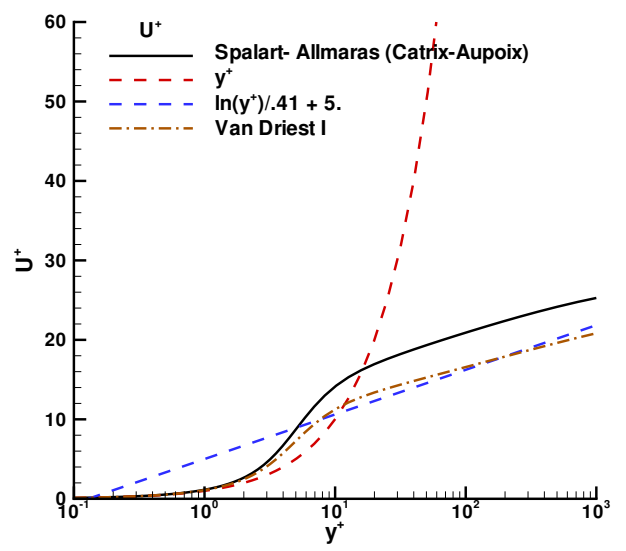

(d) 1-Eq: Spalart-Almaras (Catrix-Aupoix Compressible Model)

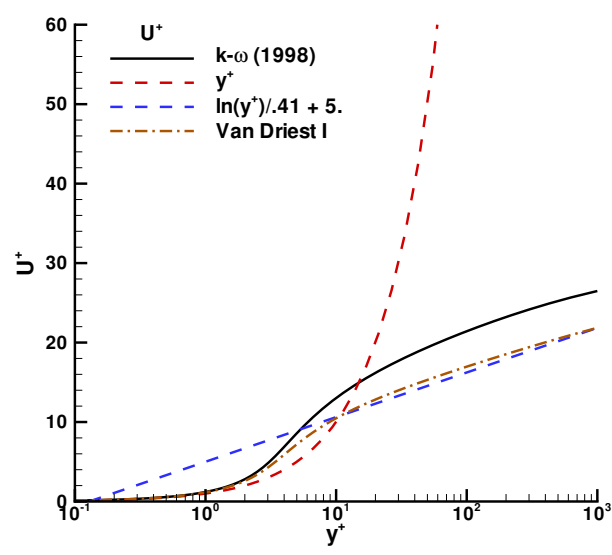

(f) 2-Eq: $k-\omega(1998)$

Figure 22. The $U^{+}$profile for various models (black line) at $x=1 \mathrm{~m}$ transformed using Van Driest theory to an incompressible profile (brown dashed dot line) with comparisons to the incompressible limits for $y^{+}<1$ (red dashed line) and $y^{+}>10$ (blue dashed line). 


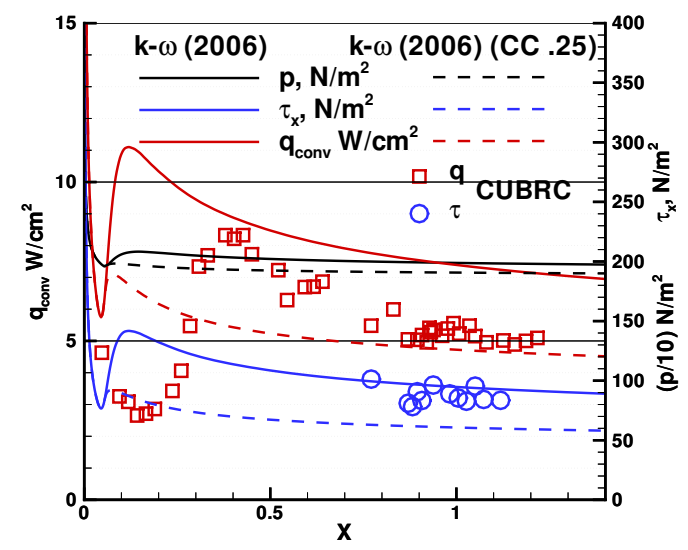

(a) $k-\omega(2006), M_{t 0}=0.25$

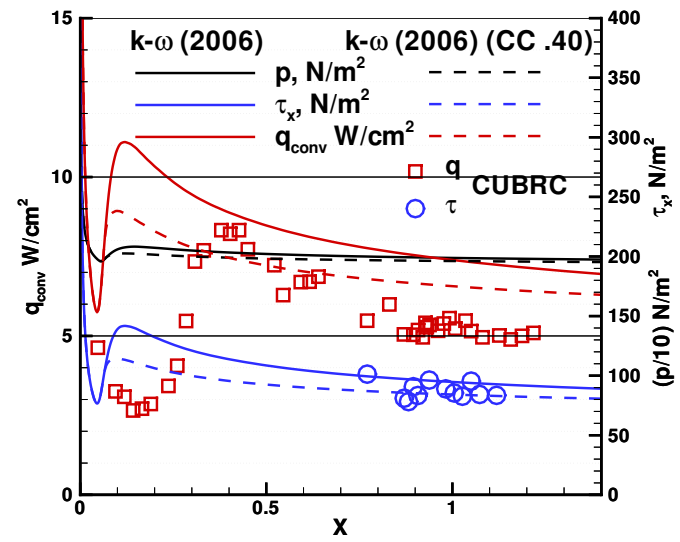

(c) $k-\omega(2006), M_{t 0}=0.40$

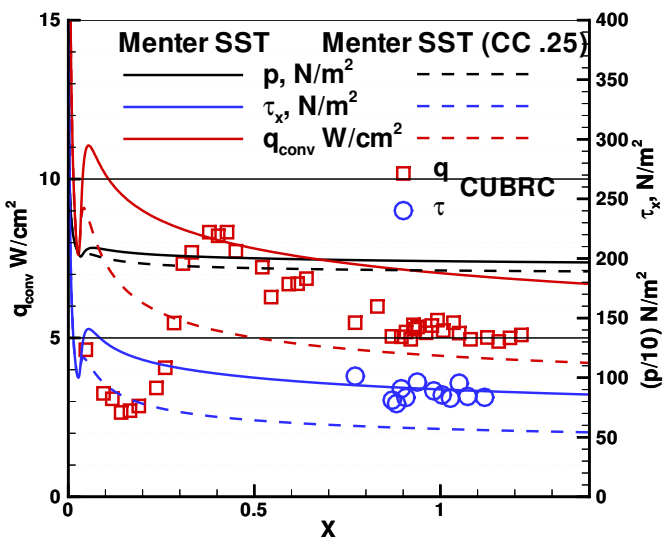

(e) Menter SST, $M_{t 0}=0.25$

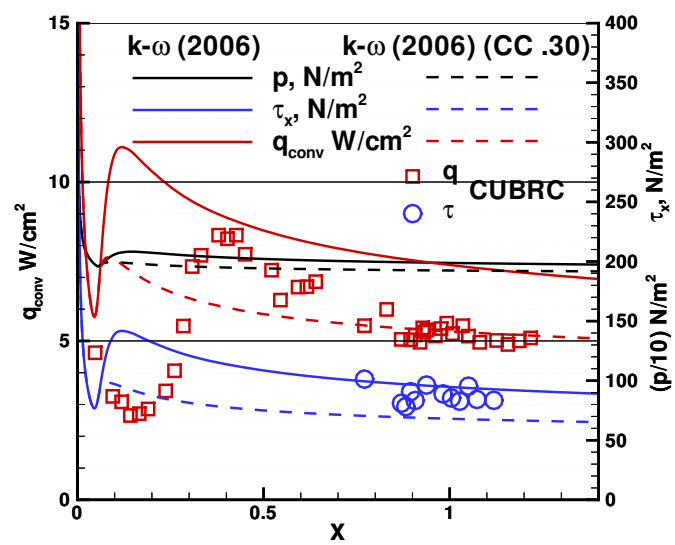

(b) $k-\omega(2006), M_{t 0}=0.30$

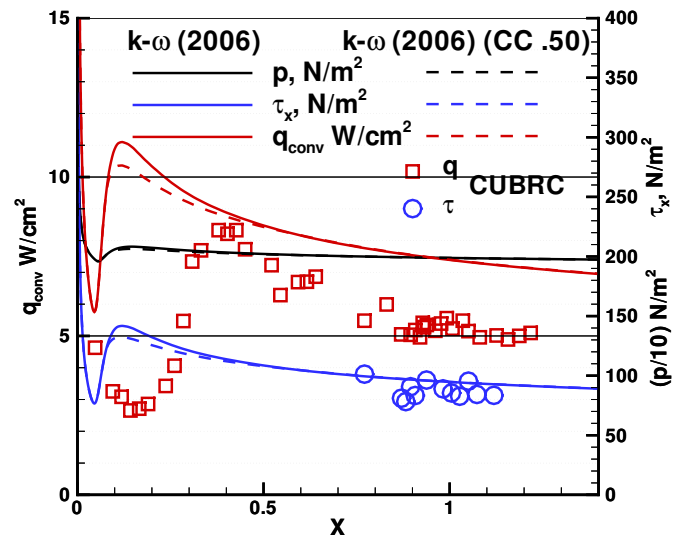

(d) $k-\omega(2006), M_{t 0}=0.50$

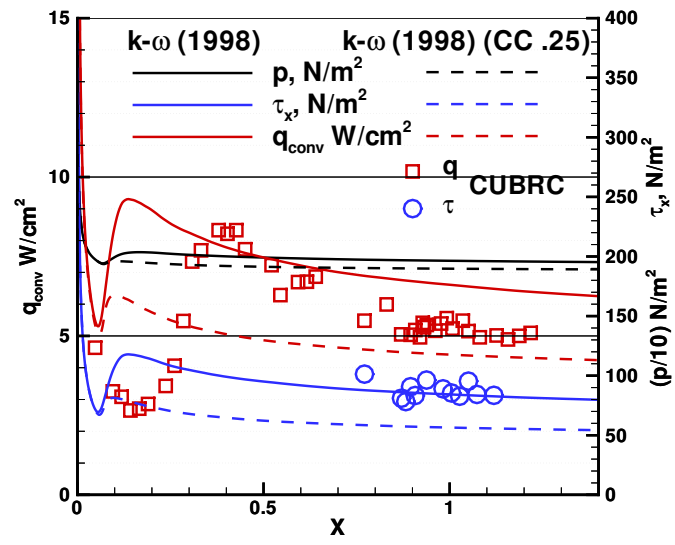

(f) $k-\omega(1998), M_{t 0}=0.25$

Figure 23. Effects of Wilcox compressibility corrections on pressure, shear, and heating distributions over a flat plate at Mach 11.1 compared to experimental data. 


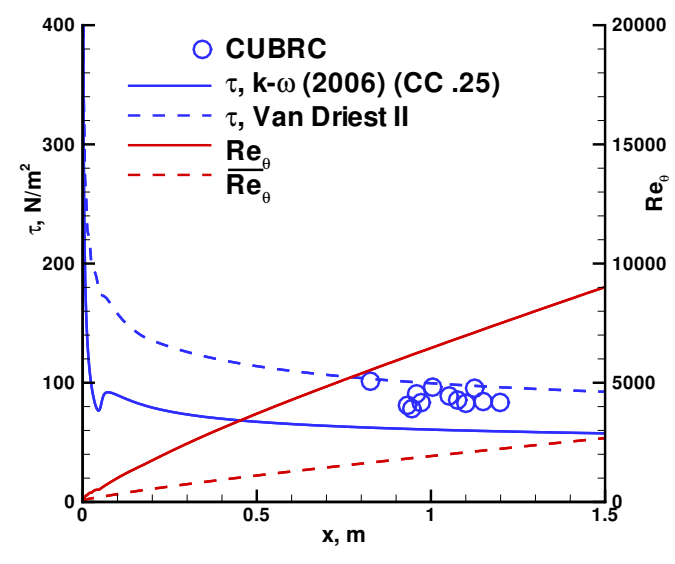

(a) $k-\omega(2006), M_{t 0}=0.25$

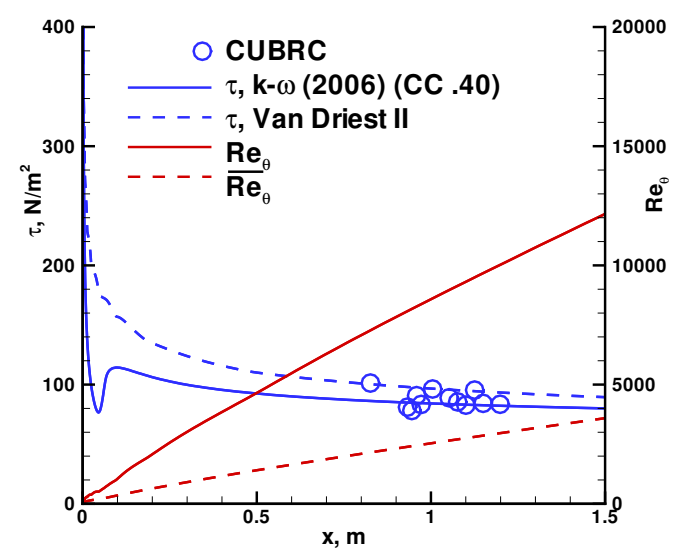

(c) $k-\omega(2006), M_{t 0}=0.40$

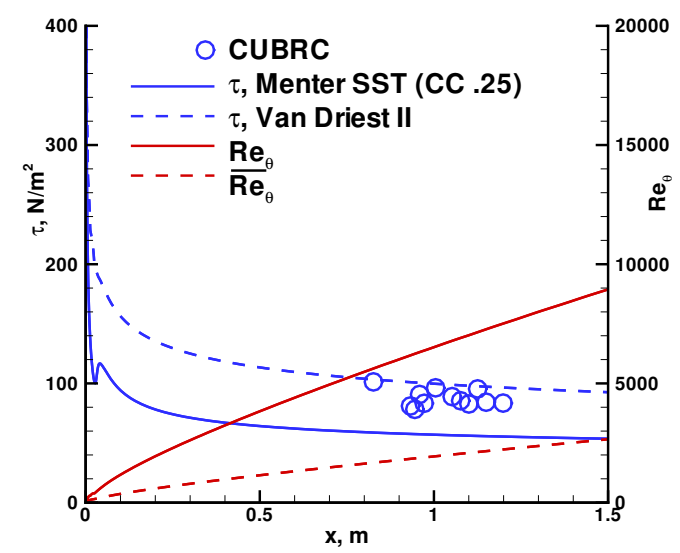

(e) Menter SST, $M_{t 0}=0.25$

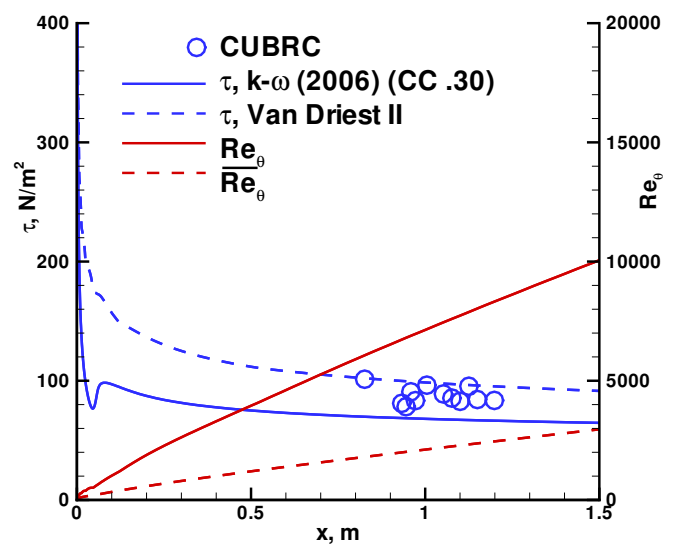

(b) $k-\omega(2006), M_{t 0}=0.30$

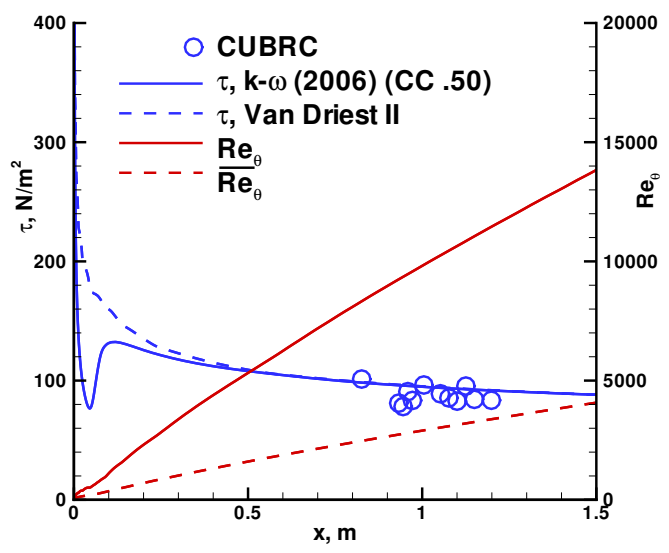

(d) $k-\omega(2006), M_{t 0}=0.50$

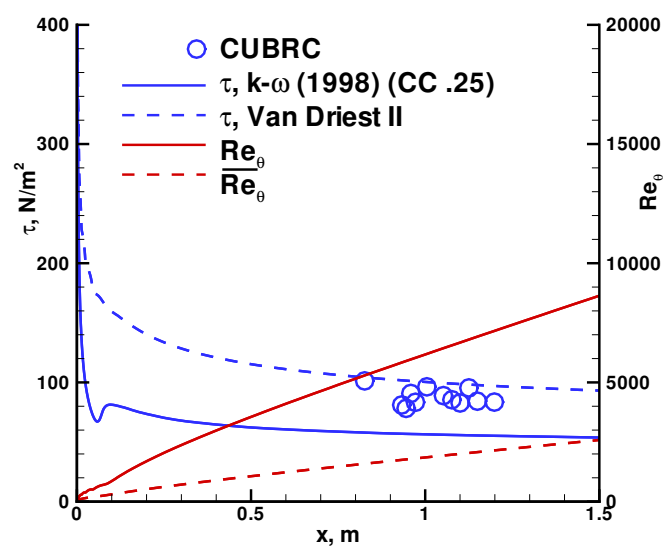

(f) $k-\omega(1998), M_{t 0}=0.25$

Figure 24. Shear distribution computed from turbulence models using Wilcox compressibility corrections compared to shear computed from Van Driest transformation. The computed momentum thickness Reynolds number distribution from each model and the transformed, incompressible momentum thickness Reynolds number used in the Van Driest transform show sensitivity to choice of turbulent Mach number cutoff parameter $M_{t 0}$. 


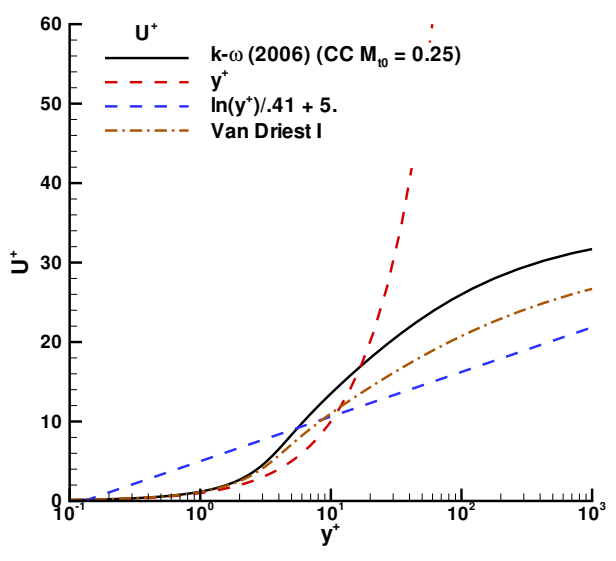

(a) $k-\omega(2006), M_{t 0}=0.25$

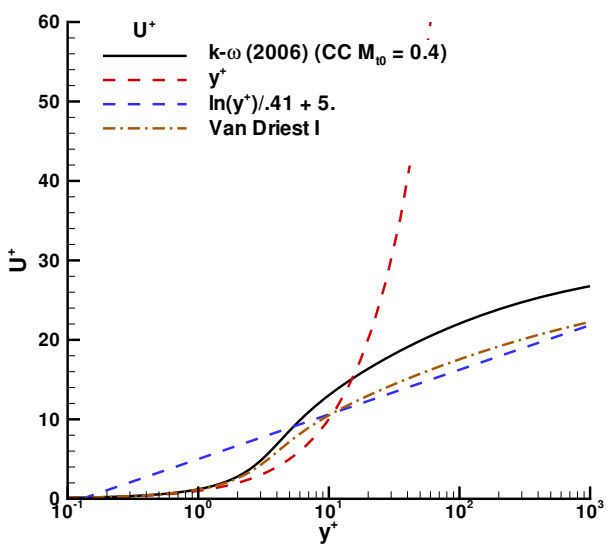

(c) $k-\omega(2006), M_{t 0}=0.40$

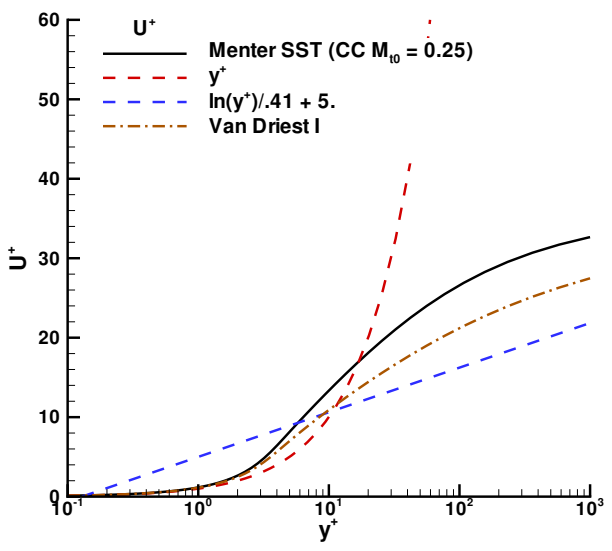

(e) Menter SST, $M_{t 0}=0.25$

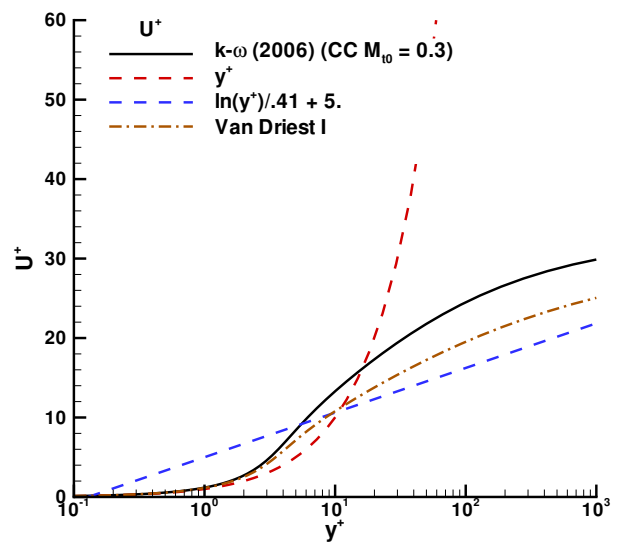

(b) $k-\omega(2006), M_{t 0}=0.30$

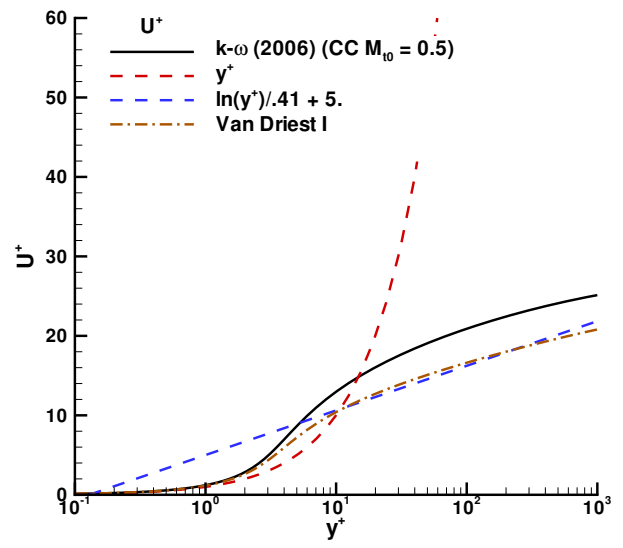

(d) $k-\omega(2006), M_{t 0}=0.50$

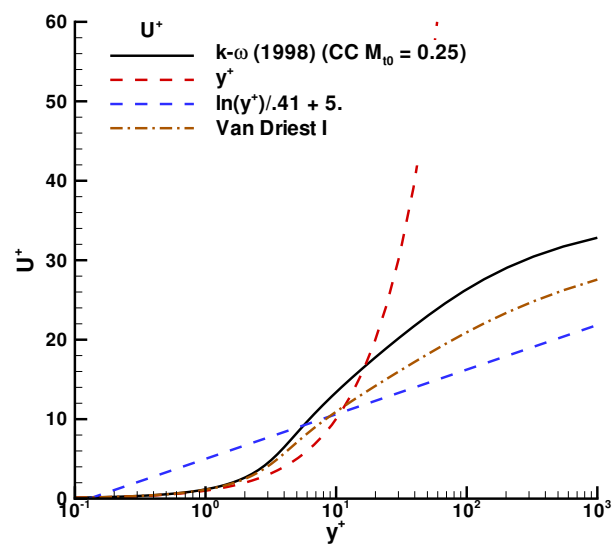

(f) $k-\omega(1998), M_{t 0}=0.25$

Figure 25. Effect of Wilcox compressibility correction on the $U^{+}$profile for various models (black line) at $x=1 \mathrm{~m}$ transformed using Van Driest theory to an incompressible profile (brown dashed dot line) with comparisons to the incompressible limits for $y^{+}<1$ (red dashed line) and $y^{+}>10$ (blue dashed line). Agreement with the incompressible limit for $y^{+}>10$ is degraded as the turbulent Mach number cutoff $M_{t 0}$ is decreased. 
Sensitivity of the simulated results to $P r_{t}$ is investigated in Fig. 26. The dashed lines in each subfigure represent the new results with $P r_{t}=1.0$ (a) and $P r_{t}=0.5$ (b). A smaller value of $P r_{t}$ corresponds to a larger value of turbulent conductivity. In the case of the flat plate, the temperature peak in the boundary layer is diffused and the surface heating is reduced when $\operatorname{Pr}_{t}$ is decreased. (See Fig. 27 (a).) In contrast, note that a lower value of the critical turbulent Mach number in the compressibility correction (Fig. 27 (b)) tends to move the temperature peak further away from the wall and lower the heating rate. In general, agreement with experimental data for heating is improved but agreement with shear is worse with $\operatorname{Pr}_{t}=0.5$ for this case.

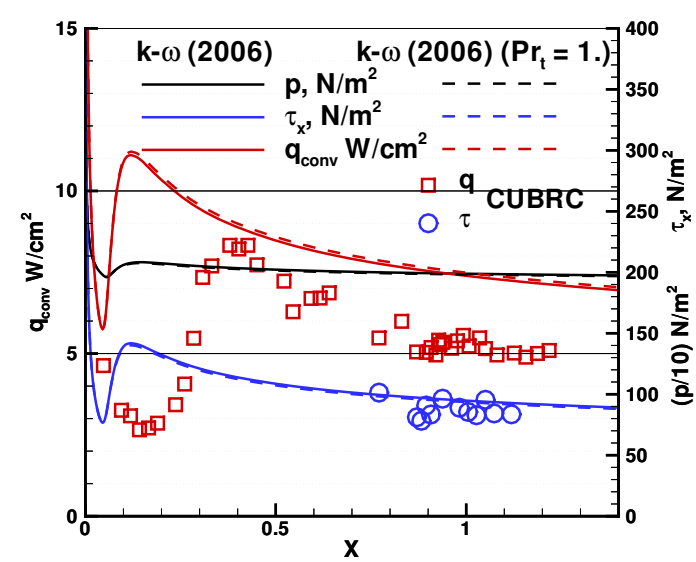

(a) $P r_{t}=1.0$

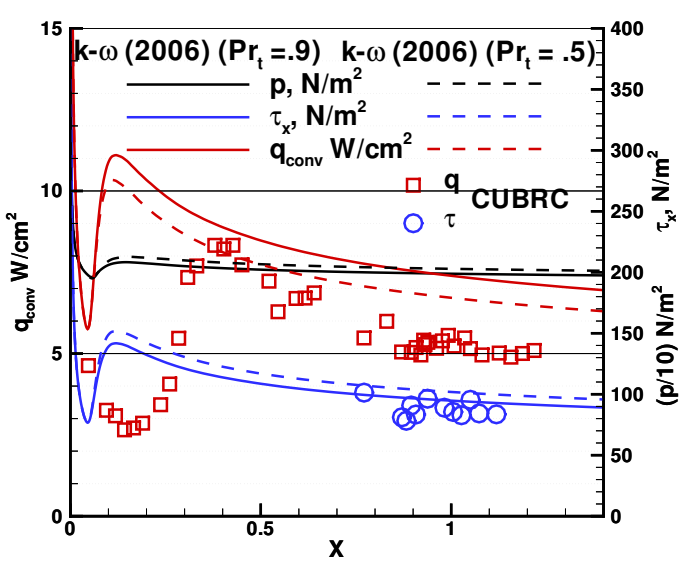

(b) $P r_{t}=0.5$

Figure 26. Effects of turbulent Prandtl number $P r_{t}$ on pressure, shear, and heating distributions over a flat plate at Mach 11.1 in the $k-\omega(2006)$ model.

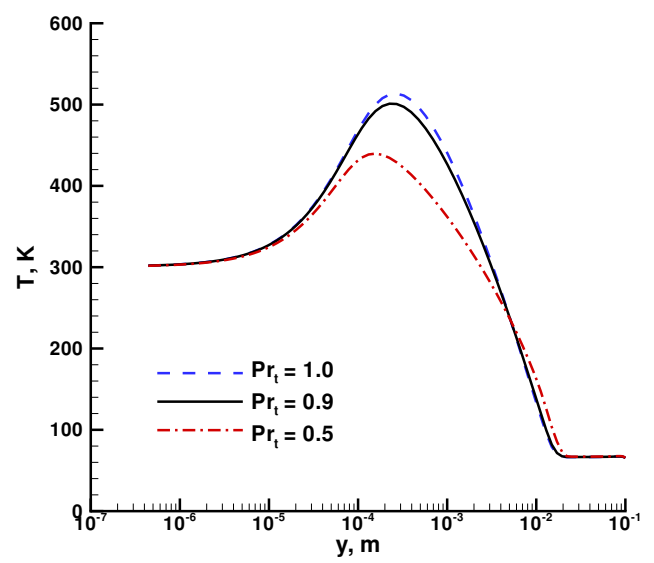

(a) $P r_{t}$ effects with no compressibility correction

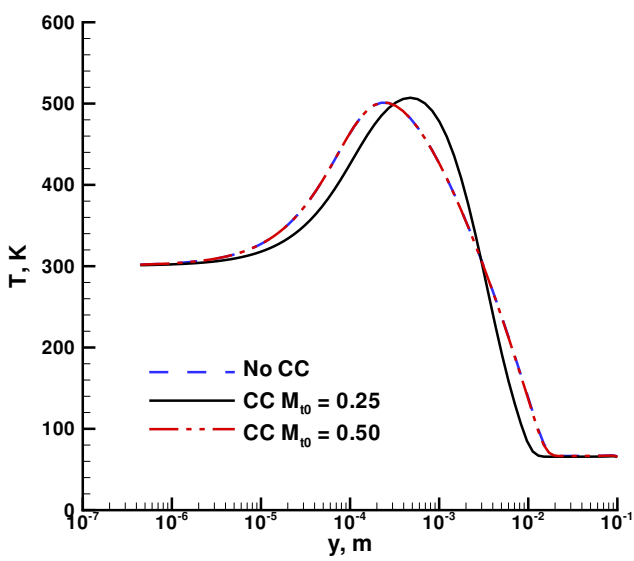

(b) Compressibility correction effects with $\operatorname{Pr}_{t}=0.9$

Figure 27. Temperature profile at $x=1 \mathrm{~m}$ as a function of turbulent Prandtl number and compressibility correction for Mach 11.1 flow over a flat plate in the $k-\omega$ (2006) model. 
The next two sets of figures on the flat plate simulations address issues of turbulent kinetic energy coupling (Fig. 28) and grid convergence (Fig. 29). In the present formulation turbulent kinetic energy, $k$, is included as part of the total energy, (i.e. $E=e+\left(u^{2}+v^{2}+w^{2}\right) / 2+k$ ). Consequently, the convective and diffusive transport of $k$ are included in the total energy conservation, Eq. 3. If one assumes that the turbulent kinetic energy content of the flow is significantly smaller than the kinetic and internal energy content everywhere then an uncoupled energy formulation may be tested with all terms involving $k$ omitted from Eq. 3 and $E=e+\left(u^{2}+v^{2}+w^{2}\right) / 2$. Plots of surface shear and heating in Fig. 28 (a) and the temperature profile at $x=1 \mathrm{~m}$ in Fig. 28 (b) with default coupled results in solid lines and uncoupled results in dashed lines show negligible effect of decoupling in this application. The same set of distributions are used to show grid convergence in Fig. 29. The baseline grid of $(300 \times 96)$ cells was decreased by a factor of 2 in both directions to $(150 \times 48)$ cells. There is almost a perfect overlay of results on the two grid systems.

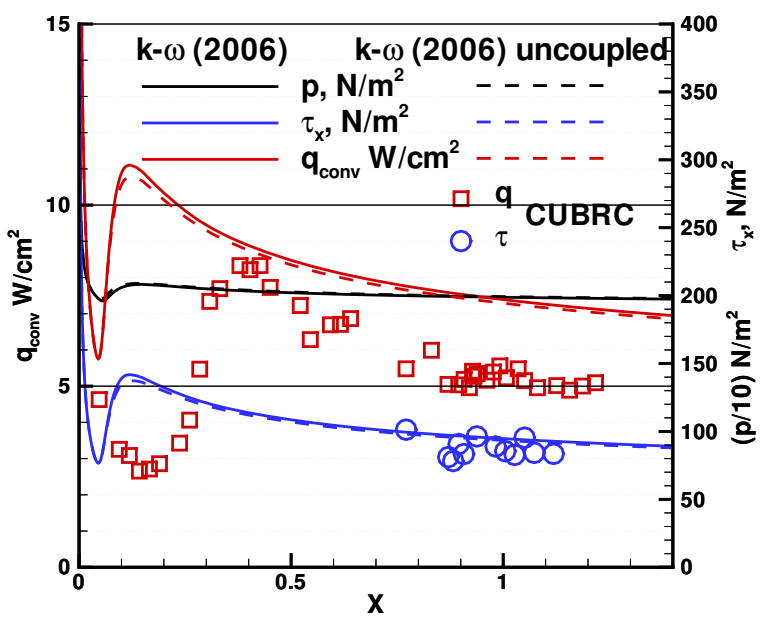

(a) Surface distributions

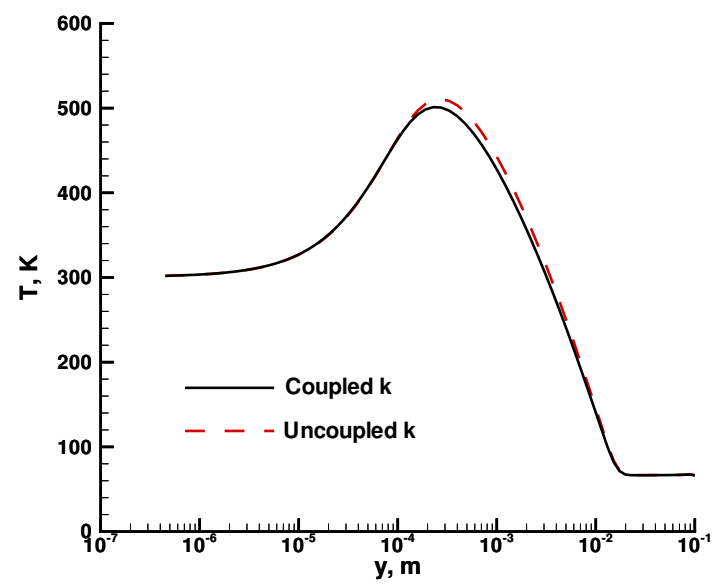

(b) Temperature profile at $x=1 \mathrm{~m}$

Figure 28. Effects of turbulent kinetic energy coupling on simulations of Mach 11.1 flow over a flat plate in the $k-\omega(2006)$ model.

Finally, we compare results from implementation of models in LAURA and DPLR (V4.01.1) in Fig. 30 using laminar, algebraic Baldwin-Lomax, Spalart Almaras, and Menter-SST models. Original implementations of the models are shown on the left (a) and more consistent implementations of the models are shown on the right (b). Simulations using laminar and algebraic turbulence models are in excellent agreement between the two codes. Small differences are noted between the 1- and 2-equation model results as originally implemented in the two codes. The difference in the 1-equation model came from an extra source term associated with different interpretations of the Catrix-Aupoix compressibility changes to the Spalart-Almaras model. The original presentation of the model was not written in strong conservation form. LAURA added a source term to enable the equation to be written in strong conservation form. DPLR coding did not alter the source terms in this way. When this extra source term was removed (resulting in Eq.20 as the new baseline) excellent agreement between implementations was achieved. The difference in the Menter-SST simulations was traced to use of 1st-order accurate implementation of convective flux for the two turbulence equations in DPLR (loosely coupled) and 2nd-order accurate implementation of convective flux for all equations in LAURA (strongly coupled). When the LAURA implementation was modified to use 1st-order flux the agreement is much improved. Note that differences in the implementation of the production term (discussed in association with Fig. 8) produced changes that were small compared to the difference in order of accuracy for this flat plate case. The laminar simulation is in good agreement with experimental data up to the transition location. After transition the algebraic turbulence model provides best agreement with experimental data for the flat plate. 


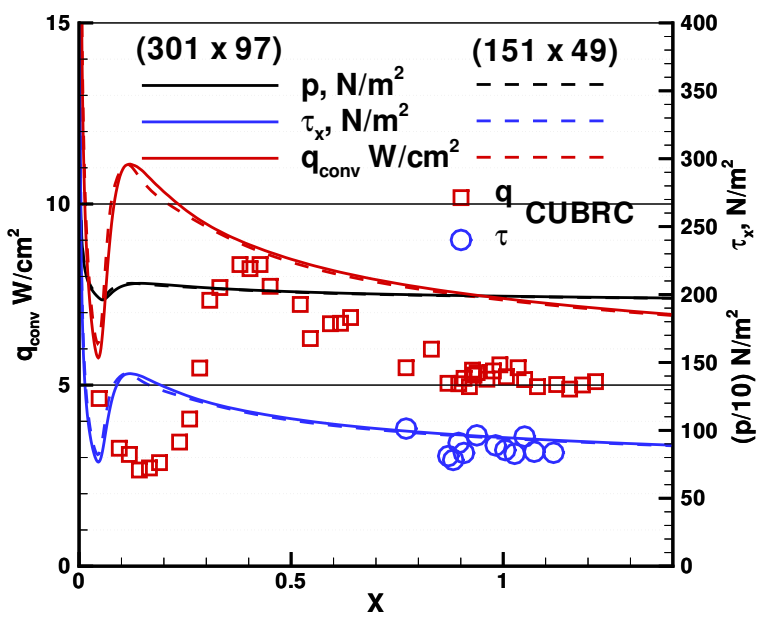

(a) Surface distributions

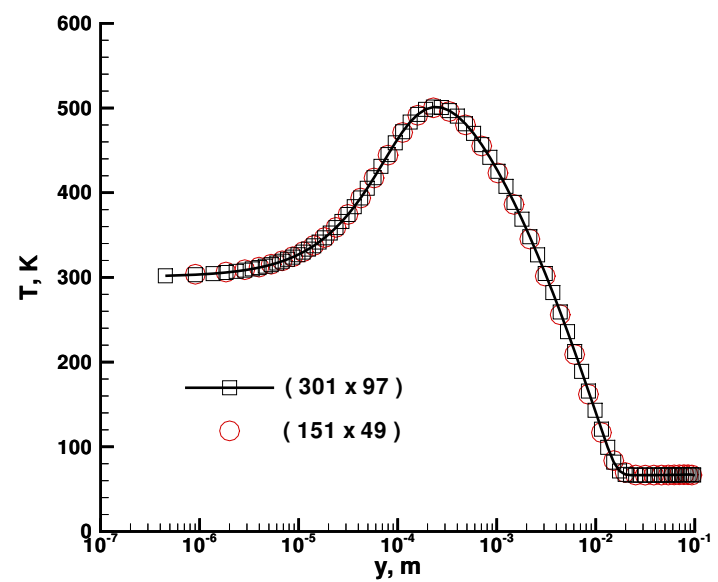

(b) Temperature profile at $x=1 \mathrm{~m}$

Figure 29. Effects of grid resolution on simulations of Mach 11.1 flow over a flat plate in the $k-\omega(2006)$ model.

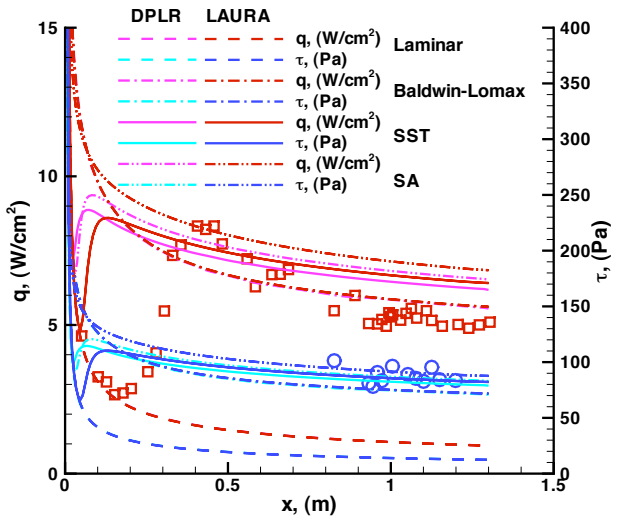

(a) Original models in LAURA and DPLR

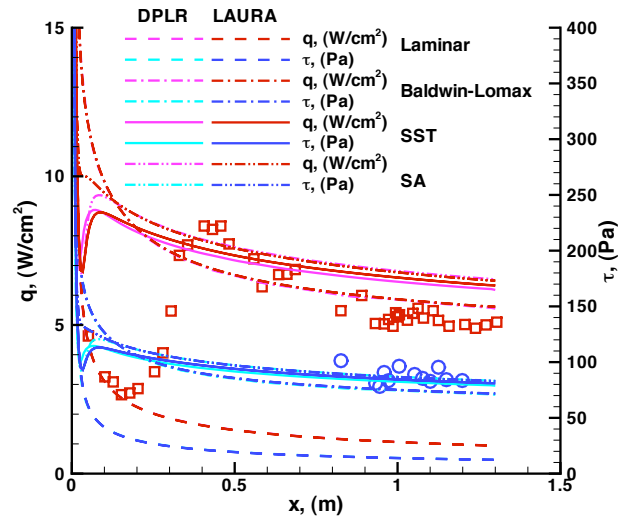

(b) Fully consistent models in LAURA and DPLR

Figure 30. Comparison of turbulence model simulations as implemented in LAURA and DPLR. 


\section{References}

${ }^{1}$ Mazaheri, A., Gnoffo, P. A., Johnston, C. O., and Kleb, B., "LAURA Users Manual: 5.3-48528," NASA TM 216836 , August 2010.

${ }^{2}$ Gnoffo, P. A., "An Upwind-Biased, Point-Implicit Relaxation Algorithm for Viscous, Compressible Perfect-Gas Flows," NASA TP 2953, Feb. 1990.

${ }^{3}$ Wright, M. J., Candler, G. V., and Bose, D., "Data-Parallel Line Relaxation Method for the Navier-Stokes Equations," AIAA J., Vol. 36, No. 9, 1998, pp. 1603-1609.

${ }^{4}$ White, J. A. and Morrison, J. H., "A Pseudo-Temporal Multi-Grid Relaxation Scheme for Solving the Parabolized Navier-Stokes Equations," AIAA Paper 99-3360, June 1999.

${ }^{5}$ Barnhardt, M. and Candler, G. V., "Detached Eddy Simulation of Hypersonic Base Flows During Atmospheric Entry," AIAA Paper 2006-3575, 2006.

${ }^{6}$ Martin, M. P. and Candler, G. V., "Subgrid-scale model for the temperature fluctuations in reacting hypersonic turbulent flows," Physics of Fluids, Vol. 11, No. 9, Sept. 1999, pp. 2765-2771.

${ }^{7} \mathrm{Wu}, \mathrm{M}$. and Martin, M. P., "Direct Numerical Simulation of Supersonic Turbulent Boundary Layer over a Compression Ramp," AIAA J., Vol. 45, No. 4, 2007, pp. 879-889.

${ }^{8}$ Smits, A. J., Martin, M. P., and Girimaji, S., "Current Status of Basic Research in Hypersonic Turbulence," AIAA Paper 2009-151, Jan. 2009.

${ }^{9}$ Johnson, H. B. and Candler, G. V., "Hypersonic Boundary Layer Stability Analysis Using PSE-Chem," AIAA Paper 2005-5023, 2005.

${ }^{10}$ Chang, C.-L., "LASTRAC.3d: Transition Prediction in 3D Boundary Layers," AIAA Paper 2004-2542, 2004.

${ }^{11}$ Lee, C. B. and Wang, S., "Study of the shock motion in a hypersonic shock system/turbulent boundary layer interaction," Experiments in Fluids, Vol. 19, No. 3, 1995, pp. 143-149.

${ }^{12}$ Roy, C. J. and Blottner, F. G., "Review and Assessment of Turbulence Models for Hypersonic Flow," Progress in Aerospace Sciences, Vol. 42, 2006, pp. 469-530.

${ }^{13}$ Settles, G. S. and Dodson, L. J., "Hypersonic shock/boundary-layer interaction database," NASA CR 177577, April 1991.

${ }^{14}$ Settles, G. S. and Dodson, L. J., "Supersonic and Hypersonic Shock/Boundary-Layer Interaction Database," AIAA J., Vol. 32, No. 7, July 1994, pp. 1377-1383.

${ }^{15}$ Knight, D., Yan, H., Panaras, A. G., and Zheltovodov, A., "Advances in CFD Prediction of Shock Wave Turbulent Boundary Layer Interactions," RTO-TR-AVT-007-V3 11, Nov. 2002.

${ }^{16}$ Reinartz, B., Ballmann, J., Brown, L., Fischer, C., and Boyce, R., "Shock Wave / Boundary Layer Interactions in Hypersonic Intake Flows," 2nd European Conference for Aerospace Sciences Session 2.14, 2007.

${ }^{17}$ Holden, M., MacLean, M., Wadhams, T., and Mundy, E., "Experimental Studies of Shock Wave/Turbulent Boundary Layer Interaction in High Reynolds Number Supersonic and Hypersonic Flows to Evaluate the Performance of CFD Codes," AIAA Paper 2010-4468, June 2010.

${ }^{18}$ Billig, F. S., "Research on Supersonic Combustion," Journal of Propulsion and Power, Vol. 9, No. 4, 1993, pp. 499-514.

${ }^{19}$ Berry, S., Daryabeigi, K., Wurster, K., and Bittner, R., "Boundary Layer Transition on X-43A," AIAA Paper 2008-3736, June 2008.

${ }^{20}$ Gupta, R. N., Lee, K. P., Moss, J. N., Zoby, E. V., and Tiwari, S. N., "Viscous Shock-Layer Analysis of Hypersonic Flows Over Long Slender Bodies," AIAA Paper 87-2487, August 1987.

${ }^{21}$ Cebeci, T. and Smith, A. M. O., "A Finite-Difference Method for Calculating Compressible Laminar and Turbulent Boundary Layers," Journal of Basic Engineering, Sept. 1970, pp. 523-535.

${ }^{22}$ Baldwin, B. S. and Lomax, H., "Thin Layer Approximation and Algebraic Model for Separated Turbulent Flows," AIAA Paper 78-257, January 1978.

${ }^{23}$ Cebeci, T., "Behavior of Turbulent Flow near a Porous Wall with Pressure Gradient," aiaaj, Vol. 8, No. 12, Dec. 1970, pp. 2152-2156.

${ }^{24}$ Spalart, P. R. and Allmaras, S. R., "A One-Equation Turbulence Model for Aerodynamic Flows," Recherche Aerospatiale, Vol. 1, No. 1, 1994, pp. 5-21.

${ }^{25}$ Catrix, S. and Aupoix, B., "Density Corrections for Turbulence Models," Aerospace Science and Technology, Vol. 4, 2000, pp. 1-11.

${ }^{26}$ Wilcox, D. C., Turbulence Modeling for CFD, Third Edition, DCW Industries, Inc., La Cañada, CA, 2006.

${ }^{27}$ Rumsey, C. L., "Compressibility Considerations for k- $\omega$ Turbulence Models in Hypersonic Boundary Layer Applications," NASA TM 215705, April 2009.

${ }^{28}$ Brown, J. L., "Turbulence Model Validation for Hypersonic Flows," AIAA Paper 2002-3308, June 2002.

${ }^{29}$ Sarkar, S., Erlebacher, G., Hussaini, M. Y., and Kreiss, H. O., "The Analysis and Modeling of Dilatational Terms in Compressible Turbulence," NASA CR 181959.

${ }^{30}$ Zeman, O., "Dilatational Dissipation: The Concept and Application in Modeling Compressible Mixing Layers," Physics of Fluids A, Vol. 2, No. 2, 1990.

${ }^{31}$ Menter, F. R., "Two-Equation Eddy-Viscosity Turbulence Models for Engineering Applications," AIAA J., Vol. 32, No. 8, Aug. 1994, pp. 1598-1605.

${ }^{32}$ Oberkampf, W. L. and Roy, C. J., Verification and Validation in Scientific Computing, Cambridge University Press, 2010.

${ }^{33}$ Holden, M. S., "Studies of the Mean and Unsteady Structure of Turbulent Boundary Layer Separation in Hypersonic Flow," AIAA Paper 91-1778, June 1991.

${ }^{34}$ Orr, M. W., Design, Analysis, and Initial Testing of a Fiber-Optic Shear Gage for 3D, High-Temperature Flows, Ph.D. thesis, Virginia Polytechnic Institute and State University, Blacksburg, Virginia, 2004. 
${ }^{35}$ Goyne, C. P., Stalker, R. J., and Paull, A., "Transducer for Direct Measurement of Skin Friction in Hypervelocity Impulse Facilities," AIAA J., Vol. 40, No. 1, 2002, pp. 42.

${ }^{36}$ Gnoffo, P. A., "CFD Validation Studies for Hypersonic Flow Prediction," AIAA Paper 2001-1025, Jan. 2001.

${ }^{37}$ MacLean, M., Private communication, 2010.

${ }^{38}$ Elfstrom, G. M., "Turbulent hypersonic flow at a wedge compression corner," J Fluid Mech, Vol. 53, No. 1, 1972, pp. 113-127.

${ }^{39}$ Coleman, G. T. and Stollery, J. L., "Heat transfer from hypersonic turbulent flow at a wedge compression corner," $J$ Fluid Mech, Vol. 56, No. 4, 1972, pp. 741-752.

${ }^{40}$ Huang, P. G. and Coakley, T. J., "Turbulence Modeling for Complex Hypersonic Flows," AIAA Paper 93-0200, 1993.

${ }^{41}$ Coratekin, T., van Keuk, J., and Ballmann, J., "Performance of Upwind Schemes and Turbulence Models in Hypersonic Flows," AIAA J., Vol. 42, No. 5, May 2004, pp. 945-957.

${ }^{42}$ Rodi, W., "Experience with two-layer models combining the k-epsilon model with a one-equation model near the wall," AIAA Paper 1991-216, Jan. 1991.

${ }^{43}$ Horstman, C. C., "Hypersonic Shock-Wave Turbulent-Boundary-Layer Interaction Flows - Experiment and Computation," AIAA Paper 1991-1760, June 1991.

${ }^{44}$ Kussoy, M. I. and Horstman, C. C., "Documentation of Two- and Three-Dimensional Hypersonic Shock Wave/Turbulent Boundary Layer Interaction Flows," NASA TM 101075, Jan 1989.

${ }^{45}$ Coleman, G. T., "A Study of Hypersonic Boundary Layers over a Family of Axisymmetric Bodies at Zero Incidence: Preliminary Report and Data Tabulation," Imperial College of Science and Technology I. C. Aero Report 73-06, Sep 1973.

${ }^{46}$ Huang, P. G. and Coleman, G. N., "Van Driest Transformation and Compressible Wall-Bounded Flows," AIAA J., Vol. 32, No. 10, Oct. 1994, pp. 2110-2112.

${ }^{47}$ Huang, P. G. and Coleman, G. N., "Errata: Van Driest Transformation and Compressible Wall-Bounded Flows," AIAA J., Vol. 33, No. 9, 1995, pp. 1756.

${ }^{48}$ Van Driest, E. R., "Turbulent Boundary Layer in Compressible Fluids," Journal of Aeronautical Science, Vol. 18, No. 3, March 1951, pp. 145-160.

${ }^{49}$ von Kármán, T., "Turbulence and Skin Friction," Journal of the Aeronautical Sciences, Vol. 1, No. 1, Jan. 1934, pp. 1-20.

${ }^{50}$ Schoenherr, K. E., "Resistance of Flat Surfaces Moving Through a Fluid," Society of Naval Architects and Marine Engineers, Vol. 40, 1932, pp. 279-313.

${ }^{51}$ Hopkins, E. J. and Inouye, M., "An Evaluation of Theories for Predicting Turbulent Skin Friction and Heat Transfer on Flat Plates at Supersonic and Hypersonic Mach Numbers," AIAA J., Vol. 9, No. 6, June 1971, pp. 993-1003.

${ }^{52}$ Van Driest, E. R., "Problems of Aerodynamic Heating," Aeronautical Engineering Review, Vol. 15, No. 10, Oct. 1956, pp. 26-41. 